\title{
Schiff Base Cobalt(II) Complex-Catalyzed Highly Markovnikov- Selective Hydrosilylation of Alkynes
}

Maciej Skrodzki, ${ }^{a, b}$ Violetta Patroniak, ${ }^{a}$ Piotr Pawluć a,b

${ }^{a}$ Faculty of Chemistry, Adam Mickiewicz University, Uniwersytetu Poznańskiego 8, 61-614 Poznań, Poland.

${ }^{\mathrm{b}}$ Centre for Advanced Technologies, Adam Mickiewicz University, Uniwersytetu Poznańskiego 10, 61-614 Poznań, Poland.

CONTENTS:

$\begin{array}{lll}\text { 1. Experimental procedures } & \text { P2 }\end{array}$

2. Analytical data of isolated products $\quad$ P4

$\begin{array}{lll}\text { 3. Deuterium labelled experiment } & \text { P14 }\end{array}$

$\begin{array}{ll}\text { 4. Spectra of products } & \text { P15 }\end{array}$

5. References P55

\section{CONTACT WITH AUTHORS:}
M. Skrodzki
maciej.skrodzki@amu.edu.pl
P. Pawluć piotr.pawluc@amu.edu.pl
V. Patroniak violapat@amu.edu.pl 


\section{EXPERIMENTAL PROCEDURES}

1.1. General remarks:

All reactions were performed in flame-dried glassware under argon atmosphere. THF was purified by distillation over sodium and benzophenone, under argon atmosphere. Other solvents were dried by distillation over calcium hydride.

Gas chromatography was performed on a Bruker Scion 436-GC with a $30 \mathrm{~m}$ Agilent VF5-ms $0.53 \mathrm{~mm}$ Megabore column and a TCD detector. The temperature program was as follows: $60^{\circ} \mathrm{C}(3 \mathrm{~min}), 20^{\circ} \mathrm{C} / \mathrm{min}, 280^{\circ} \mathrm{C}$ (20 min). Decane was used as a reference. GC-MS analyses were performed on a Bruker Scion 436-GC with a $30 \mathrm{~m}$ Varian DB-5 $0.25 \mathrm{~mm}$ capillary column and a Scion SQ-MS mass spectrometry detector. The temperature program was as follows: $60^{\circ} \mathrm{C}(3 \mathrm{~min}), 10^{\circ} \mathrm{C} / \mathrm{min}, 250{ }^{\circ} \mathrm{C}$ (15 min). NMR analyses were performed on a Bruker Fourier $300 \mathrm{MHz}$ or $400 \mathrm{MHz}$ spectrometer.

FT-IR spectra were recorded on a Nicolet iS50 (Thermo Scientific) Fourier transform spectrophotometer equipped with a diamond ATR unit. In all cases, 16 scans at a resolution of $2 \mathrm{~cm}^{-1}$ were collected, to record the spectra in the range of $4000-400 \mathrm{~cm}^{-1}$.

HRMS Spectra were recorded on a QTOF type mass spectrometer (Impact HD, Bruker) in positive ion mode.

1.2. General procedure of alkyne hydrosilylation:

General procedure for hydrosilylation of alkynes A: To a flame-dried Schlenk bomb flask charged with argon, precatalyst $(0.1 \mathrm{~mol} \%)$, THF $(0.5 \mathrm{~mL})$, silane $(1.0 \mathrm{mmol})$, and alkyne $(1.0 \mathrm{mmol})$ were placed. Mixture was then heated to $40^{\circ} \mathrm{C}$ with stirring in oil bath. After 10 minutes $\mathrm{LiHBEt}_{3}$ was added $(0.3 \mathrm{~mol} \%)$, and reaction vessel was then closed. After 20 hours, hexane was added to reaction mixture. Solution was then filtered through silica plug, and concentrated on rotavapor. The crude mixture was purified by evaporating volatiles on vacuum line followed by extraction with hexane. Regioselectivity was monitored by ${ }^{1} \mathrm{H}$ NMR.

General procedure for hydrosilylation of alkynes B: To a flame-dried Schlenk bomb flask charged with argon, precatalyst $(0.5 \mathrm{~mol} \%)$, THF $(0.5 \mathrm{~mL})$, silane $(1.0 \mathrm{mmol})$, and alkyne $(1.0 \mathrm{mmol})$ were placed. Mixture was then heated to $60^{\circ} \mathrm{C}$ with stirring in oil bath. After 10 minutes $\mathrm{LiHBEt}_{3}$ was added (1.5 mol \%), and reaction vessel was then closed. After 20 hours, hexane was added to reaction mixture. Solution was then filtered through silica plug, and concentrated on rotavapor. The crude mixture was purified by evaporating volatiles on vacuum line followed by extraction with hexane. Regioselectivity was monitored by ${ }^{1} \mathrm{H}$ NMR.

General procedure for hydrosilylation of alkynes C: To a flame-dried Schlenk bomb flask charged with argon, precatalyst $(0.05 \mathrm{~mol} \%)$, THF $(0.5 \mathrm{~mL})$, silane $(1.0 \mathrm{mmol})$, and alkyne $(1.0 \mathrm{mmol})$ were placed. Mixture was then heated to $40^{\circ} \mathrm{C}$ with stirring in oil bath. After 10 minutes $\mathrm{LiHBEt}_{3}$ was added $(0.15 \mathrm{~mol} \%)$, and reaction vessel was then closed. After 20 hours, hexane was added to reaction mixture. Solution was then filtered through silica plug, and concentrated on rotavapor. The crude mixture was purified by evaporating volatiles on vacuum line followed by extraction with hexane. Regioselectivity was monitored by ${ }^{1} \mathrm{H}$ NMR.. 
1.3. Optimization of hydrosilylation of phenylacetylene with phenylsilane ${ }^{a}$

\begin{tabular}{|c|c|c|c|c|c|}
\hline & $\mathrm{Ph} \equiv+$ & $\mathrm{SiH}_{3}$ & $\begin{array}{l}\underset{\mathrm{M} \text { THF }}{(3 \times \mathrm{mol} \%)} \\
\underset{3}{(})\end{array}$ & ${ }_{3 a}{ }_{3 i P h H}+$ & \\
\hline Entry & $\begin{array}{l}\text { Catalyst } 1 \\
\text { loading [mol\%] }\end{array}$ & Time [h] & $\begin{array}{l}\text { Temp. } \\
{\left[{ }^{\circ} \mathrm{C}\right]}\end{array}$ & $\begin{array}{l}\text { Phenylsilane } \\
\text { Conversion }^{\mathrm{b}}[\%]\end{array}$ & $\begin{array}{l}\text { Selectivityc } \\
(3 a / 3 a a)\end{array}$ \\
\hline 1 & 0.1 & 20 & 40 & $>99$ & $78: 22$ \\
\hline 2 & 0.05 & 20 & 40 & $>99$ & $79: 21$ \\
\hline $3^{d}$ & 0.05 & 20 & 40 & $>99$ & $91: 9$ \\
\hline 4 & 0.05 & 20 & 20 & 91 & $79: 21$ \\
\hline 5 & 0.025 & 20 & 40 & $>99$ & $84: 16$ \\
\hline 6 & 0.01 & 20 & 40 & $>99$ & $86: 14$ \\
\hline 7 & 0.01 & 0.166 & 40 & 14 & $94: 6$ \\
\hline 8 & 0.005 & 20 & 40 & 85 & $89: 11$ \\
\hline 9 & 0.005 & 0.5 & 40 & 20 & $92: 8$ \\
\hline
\end{tabular}

${ }^{a}$ Conditions: phenylacetylene $(1 \mathrm{mmol})$, phenylsilane $(1 \mathrm{mmol}) .{ }^{\mathrm{b}, \mathrm{c} C}$ Calculated by $\mathrm{GC}$ with decane as internal standard.

${ }^{\mathrm{d}}$ Reaction with $1.5 \mathrm{mmol}$ of phenylsilane.

1.4. Ligand(L) and complex 1 synthesis:

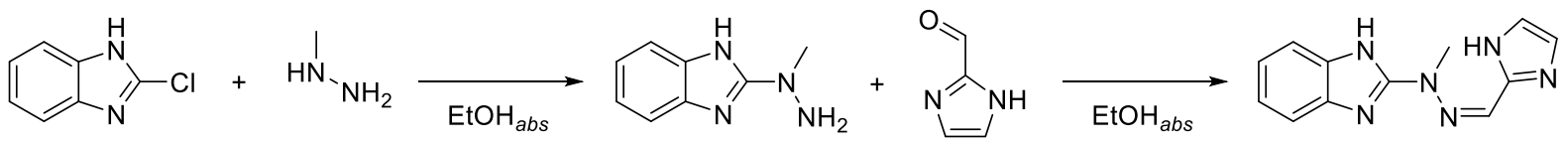

Ligand was synthesized according to reported procedure[1]. 2-chlorobenzimidazole $(8.00 \mathrm{~g}$ $0.05 \mathrm{~mol}$ ) was placed under argon atmosphere in two-necked round-bottomed flask. Methylhydrazine (11.9 g, $0.25 \mathrm{~mol}$ ), in five-fold excess, was dissolved in anhydrous ethanol and was added to the reaction mixture, which was heated in heating mantle to $80^{\circ} \mathrm{C}$ and stirred for $20 \mathrm{~h}$. The white, crystalline product was filtered on Büchner funnel and dried under vacuum. Yield $68.2 \%$ (5.8 g, $0.036 \mathrm{~mol})$. Then condensation of 2-imidazolecarboxyaldehyde with 2-(1-methylhydrazine)benzimidazole was performed. At two-necked round-bottomed flask 2-(1- methylhydrazine)benzimidazole $(1 \mathrm{~g}, 6.16 \mathrm{mmol})$ placed under argon atmosphere. The 4-imidazolecarboxyaldehyde $(0.591 \mathrm{~g}, 6.16 \mathrm{mmol})$ was dissolved in anhydrous ethanol and was added to the reaction mixture. Reaction mixture was stirred for $24 \mathrm{~h}$ at $60^{\circ} \mathrm{C}$. Yellow clear solution was cooled to room temperature. Precipitate, was then filtered by vacuum filtration, washed with anhydrous ethanol and dried under vacuum to give $1.24 \mathrm{~g}(5.16 \mathrm{mmol})$ of $\mathbf{L}$. The supernatant was concentrated to minimal amount of volume and the next part of precipitate was obtained $0.140 \mathrm{~g}(0.58 \mathrm{mmol})$. Total yield is $91 \%(1.38 \mathrm{~g}, 5.74 \mathrm{mmol})$.

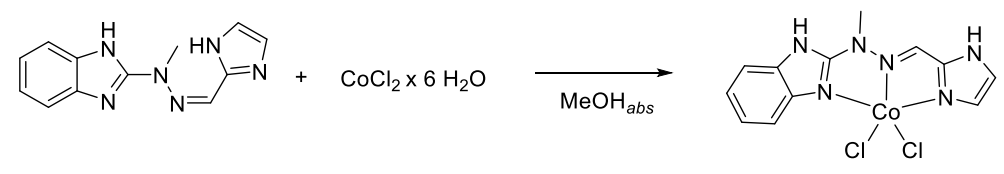

Complex 1 was synthesized according to reported procedure[2]. To a solution of $\mathbf{L}$ (100.0 $\mathrm{mg}$ ) in hot $\mathrm{MeOH}$, the methanolic solution of $\mathrm{CoCl}_{2} \cdot 6 \mathrm{H}_{2} \mathrm{O}(99.1 \mathrm{mg})$ was added. Mixture was stirred for $24 \mathrm{~h}$ at room temperature. Solvents were subsequently evaporated under vacuum to minimal amount. Then, excess of $\mathrm{Et}_{2} \mathrm{O}$ was added. Precipitate was filtered and washed twice with $\mathrm{Et}_{2} \mathrm{O}$ and air dried, affording $153.9 \mathrm{mg}$ (99.8\% yield) of complex 1. 


\section{Analytical data of isolated products}

2.1.(2a) Diphenyl(1-phenylvinyl)silane - prepared from phenylacetylene $(1.0 \mathrm{mmol})$ and diphenylsilane $(1.0 \mathrm{mmol})$ according to general procedure A. Purified by extraction with $n$ hexane followed by filtration through silica plug. Volatiles were removed under lower pressure affording pure product (colorless oil, $285.9 \mathrm{mg}->99 \%$ ).<smiles>C=C(c1ccccc1)[SiH](c1ccccc1)c1ccccc1</smiles>

${ }^{1} \mathrm{H}$ NMR $\left(300 \mathrm{MHz}, \mathrm{CDCl}_{3}\right): \delta 7.52-7.45(\mathrm{~m}, 4 \mathrm{H}), 7.36-7.23(\mathrm{~m}, 8 \mathrm{H})$, 7.21-7.12 (m, 3H), $6.23-6.19(\mathrm{~d}, 1 \mathrm{H}, J=1.7 \mathrm{~Hz}), 5.63-5.59(\mathrm{~d}, 1 \mathrm{H}, J=$ $2.4 \mathrm{~Hz}), 5.31(\mathrm{~s}, 1 \mathrm{H})$

${ }^{13} \mathrm{C} \mathrm{NMR}\left(75 \mathrm{MHz}, \mathrm{CDCl}_{3}\right): \delta 145.9,142.9,135.8,133.1,132.2,129.9$, $128.5,128.1,127.1,126.8$

Corresponds to previously reported data [3]

2.2.(2b) (1-(4-(tert-butyl)phenyl)vinyl)diphenylsilane - prepared from (4-tert-butyl)phenylacetylene $(1.0 \mathrm{mmol})$ and diphenylsilane $(1.0 \mathrm{mmol})$ according to general procedure A. Purified by extraction with $n$-hexane followed by filtration through silica plug. Volatiles were removed under lower pressure affording pure product (pale yellow oil, $340.5 \mathrm{mg}$<smiles>C=C(c1ccc(C(C)(C)C)cc1)[SiH](c1ccccc1)c1ccccc1</smiles>
- >99\%).

${ }^{1} \mathrm{H}$ NMR $\left(300 \mathrm{MHz}, \mathrm{CDCl}_{3}\right): \delta 7.53-7.46(\mathrm{~m}, 4 \mathrm{H}), 7.36-7.17(\mathrm{~m}$, $10 \mathrm{H}), 6.23-6.19(\mathrm{dd}, 1 \mathrm{H}, J=2.3 \mathrm{~Hz}), 5.58-5.53(\mathrm{~d}, 1 \mathrm{H}, J=2.4$ $\mathrm{Hz}), 5.32(\mathrm{~s}, 1 \mathrm{H}), 1.20(\mathrm{~s}, 9 \mathrm{H})$

${ }^{13} \mathrm{C}$ NMR $\left(75 \mathrm{MHz}, \mathrm{CDCl}_{3}\right): \delta 150.1,145.1,139.8,135.8,133.3$, $131.5,139.8,128.1,126.3,125.4,34.5,31.3$

Corresponds to previously reported data [3]

2.3. (2c) (1-(4-bromophenyl)vinyl)diphenylsilane - prepared from (4-bromo)phenylacetylene $(1.0 \mathrm{mmol})$ and diphenylsilane $(1.0 \mathrm{mmol})$ according to general procedure $\mathbf{B}$. Purified by extraction with $n$-hexane followed by filtration through silica plug. Volatiles were removed under lower pressure affording pure product (yellow oil, $365.0 \mathrm{mg}->99 \%$ ).<smiles>C=C(c1ccc(Br)cc1)[SiH](c1ccccc1)c1ccccc1</smiles>

${ }^{1} \mathrm{H}$ NMR $\left(300 \mathrm{MHz}, \mathrm{CDCl}_{3}\right): \delta 7.59-7.53(\mathrm{~m}, 4 \mathrm{H}), 7.47-7.34(\mathrm{~m}$, $8 \mathrm{H}), 7.24-7.16(\mathrm{~m}, 2 \mathrm{H}), 6.29-6.26(\mathrm{~d}, 1 \mathrm{H}, J=1.7 \mathrm{~Hz}), 5.74-5.70(\mathrm{~d}$, $1 \mathrm{H}, J=2.2 \mathrm{~Hz}), 5.38(\mathrm{~s}, 1 \mathrm{H})$

${ }^{13} \mathrm{C}$ NMR $\left(75 \mathrm{MHz}, \mathrm{CDCl}_{3}\right): \delta 144.9,141.9,135.8,132.7,132.6$, $131.5,130.0,128.4,128.2,121.1$

Corresponds to previously reported data [3] 
2.4.(2d) (1-cyclohex-1-enylvinyl)diphenylsilane - prepared from cyclohexylacetylene (0.5 $\mathrm{mmol})$ and diphenylsilane $(0.5 \mathrm{mmol})$ according to general procedure A. Purified by extraction with $n$-hexane followed by filtration through silica plug. Volatiles were removed under lower pressure affording pure product (yellow oil, $146.2 \mathrm{mg}->99 \%$ ).<smiles>C=C(C1=CCCCC1)[SiH](c1ccccc1)c1ccccc1</smiles>

${ }^{1} \mathrm{H}$ NMR $\left(300 \mathrm{MHz}, \mathrm{CDCl}_{3}\right): \delta 7.59-7.53(\mathrm{~m}, 4 \mathrm{H}), 7.41-7.34(\mathrm{~m}, 6 \mathrm{H})$, $5.99(\mathrm{~s}, 1 \mathrm{H}), 5.96-5.91(\mathrm{t}, 1 \mathrm{H}), 5.38-5.34(\mathrm{~d}, 1 \mathrm{H}, \mathrm{J}=2.2 \mathrm{~Hz}), 5.33-$ $5.30(\mathrm{~s}, 1 \mathrm{H}), 2.29-2.20(\mathrm{~m}, 2 \mathrm{H}), 2.10-2.01(\mathrm{~m}, 2 \mathrm{H}), 1.75-1.65(\mathrm{~m}, 2 \mathrm{H})$, $1.60-1.52(\mathrm{~m}, 2 \mathrm{H})$

${ }^{13} \mathrm{C}$ NMR $\left(75 \mathrm{MHz}, \mathrm{CDCl}_{3}\right): \delta 145.9,138.5,135.8,135.5,133.9,129.5$, $127.9,127.0,26.0,26.0,22.3,22.2$

Corresponds to previously reported data [4]

2.5.(2e) Diphenyl(3-phenylprop-1-en-2-yl)silane - prepared from 3-phenylprop-1-yne (1.0 $\mathrm{mmol})$ and diphenylsilane $(1.0 \mathrm{mmol})$ according to general procedure A. Purified by extraction with $n$-hexane followed by filtration through silica plug. Volatiles were removed under lower pressure affording pure product (yellow oil, 298,0 mg - >99\%).<smiles>C=C(Cc1ccccc1)[SiH](c1ccccc1)c1ccccc1</smiles>

${ }^{1} \mathrm{H}$ NMR $\left(300 \mathrm{MHz}, \mathrm{CDCl}_{3}\right): \delta 7.46-7.41(\mathrm{~m}, 4 \mathrm{H}), 7.33-7.24(\mathrm{~m}, 6 \mathrm{H})$, $7.19-7.09(\mathrm{~m}, 3 \mathrm{H}), 7.01-6.96(\mathrm{~d}, 2 \mathrm{H}), 5.68-5.65(\mathrm{~d}, 1 \mathrm{H}, J=1.1 \mathrm{~Hz})$, $5.49-5.46(\mathrm{~d}, 1 \mathrm{H}, \mathrm{J}=1.3 \mathrm{~Hz}), 4.92(\mathrm{~s}, 1 \mathrm{H}), 3.44(\mathrm{~s}, 2 \mathrm{H})$

${ }^{13} \mathrm{C} \mathrm{NMR}\left(75 \mathrm{MHz}, \mathrm{CDCl}_{3}\right): \delta 145.8,139.3,135.7,132.9,131.2,129.75$, $129.5,128.3,128.0,126.1,43.0$

HRMS (ESI): calculated for $\left[\mathrm{C}_{21} \mathrm{H}_{20} \mathrm{SiNa}\right]^{+}$requires $323.1226 \mathrm{~m} / \mathrm{z}$, found $323.1226 \mathrm{~m} / \mathrm{z}$

2.6.(2f) (1-(4-methoxyphenyl)vinyl)diphenylsilane - prepared from 4-ethynylanisole (1.0 mmol) and diphenylsilane $(1.0 \mathrm{mmol})$ according to general procedure A. Purified by extraction with $n$ hexane followed by filtration through silica plug. Volatiles were removed under lower pressure affording pure product (yellow oil, $313.8 \mathrm{mg}$ - >99\%).<smiles>C=C(c1ccc(OC)cc1)[SiH](c1ccccc1)c1ccccc1</smiles>

${ }^{1} \mathrm{H}$ NMR $\left(300 \mathrm{MHz}, \mathrm{CDCl}_{3}\right): \delta 7.63-7.57(\mathrm{~m}, 4 \mathrm{H}), 7.45-7.31(\mathrm{~m}$, $8 \mathrm{H}), 6.86-6.80(\mathrm{~d}, 2 \mathrm{H}), 6.28-6.25(\mathrm{~d}, 1 \mathrm{H}, J=1.8 \mathrm{~Hz}), 5.64-5.61$ $(\mathrm{d}, 1 \mathrm{H}, J=2.1 \mathrm{~Hz}), 5.42(\mathrm{~s}, 1 \mathrm{H}), 3.77(\mathrm{~s}, 3 \mathrm{H})$

${ }^{13} \mathrm{C}$ NMR $\left(75 \mathrm{MHz}, \mathrm{CDCl}_{3}\right): \delta 158.9,144.7,135.8,133.2,129.8$, $128.1,127.8,113.8,55.3$

Corresponds to previously reported data [3]

2.7.(2g) (1-cyclohexylvinyl)diphenylsilane - prepared from cyclohexylacetylene $(0.5 \mathrm{mmol})$ and diphenylsilane $(0.5 \mathrm{mmol})$ according to general procedure B. Purified by extraction with $n$ hexane followed by filtration through silica plug. Volatiles were removed under lower pressure affording pure product (yellow oil, $146.2 \mathrm{mg}->99 \%$ ).<smiles></smiles>

${ }^{1} \mathrm{H}$ NMR $\left(300 \mathrm{MHz}, \mathrm{CDCl}_{3}\right): \delta 7.62-7.56(\mathrm{~m}, 4 \mathrm{H}), 7.46-7.36(\mathrm{~m}, 6 \mathrm{H})$, $5.94-5.92(\mathrm{~m}, 1 \mathrm{H}), 5.46-5.42(\mathrm{~d}, 1 \mathrm{H}, J=2.3 \mathrm{~Hz}), 5.18(\mathrm{~s}, 1 \mathrm{H}), 2.25-$ $2.15(\mathrm{~m}, 1 \mathrm{H}), 1.81-1.71(\mathrm{~m}, 4 \mathrm{H}), 1.31-1.17(\mathrm{~m}, 4 \mathrm{H}), 1.20-1.09(\mathrm{~m}$, $2 \mathrm{H})$

${ }^{13} \mathrm{C}$ NMR $\left(75 \mathrm{MHz}, \mathrm{CDCl}_{3}\right): \delta 152.0,135.7,133.9,129.5,128.0,127.9$, $42.9,33.2,26.7,26.2$

Corresponds to previously reported data [4] 
2.8.(2h) (1-cyclopropylvinyl)diphenylsilane - prepared from cyclopropylacetylene (1.0 mmol) and diphenylsilane $(1.0 \mathrm{mmol})$ according to general procedure A. Purified by extraction with $n$ hexane followed by filtration through silica plug. Volatiles were removed under lower pressure affording pure product (yellow oil, $249.8 \mathrm{mg}$ - >99\%).<smiles>C=C(C1CC1)[Si](c1ccccc1)c1ccccc1</smiles>

${ }^{1} \mathrm{H}$ NMR $\left(300 \mathrm{MHz}, \mathrm{CDCl}_{3}\right): \delta 7.64-7.57(\mathrm{~m}, 4 \mathrm{H}), 7.45-7.34(\mathrm{~m}, 6 \mathrm{H}), 5.65$ $-5.62(\mathrm{~d}, 1 \mathrm{H}, J=1.8 \mathrm{~Hz}), 5.28-5.25(\mathrm{~d}, 1 \mathrm{H}, J=2.4 \mathrm{~Hz}), 5.01(\mathrm{~s}, 1 \mathrm{H}), 1.59$ $-1.50(\mathrm{~m}, 1 \mathrm{H}), 0.73-0.64(\mathrm{~m}, 2 \mathrm{H}), 0.57-0.51(\mathrm{~m}, 2 \mathrm{H})$

${ }^{13} \mathrm{C}$ NMR $\left(75 \mathrm{MHz}, \mathrm{CDCl}_{3}\right): \delta 147.7,135.5,133.1,129.5,127.5,127.8$, $125.8,16.4,7.3$

Corresponds to previously reported data [5]

2.9.(2i) (E)-diphenyl(1-phenylprop-1-en-1-yl)silane (major product) - prepared from 1phenylprop-1-yne $(0.5 \mathrm{mmol})$ and diphenylsilane $(0.5 \mathrm{mmol})$ according to general procedure B. Purified by extraction with $n$-hexane followed by filtration through silica plug. Volatiles were removed under lower pressure affording pure product (yellow oil, $146.2 \mathrm{mg}$ - >99\%).<smiles>C/C=C(\c1ccccc1)[SiH](c1ccccc1)c1ccccc1</smiles>

Major product<smiles>CC(=Cc1ccccc1)[SiH](c1ccccc1)c1ccccc1</smiles>

${ }^{13} \mathrm{C}$ NMR $\left(75 \mathrm{MHz}, \mathrm{CDCl}_{3}\right): \delta 142.8,142.1,141.1,139.04,137.9,135.8$, $135.8,133.6,133.2,129.8,129.6,129.1,128.5,128.1,128.1,128.1$, $127.9,127.0,125.8,17.5,16.4$

Corresponds to previously reported data [3]

Minor product

2.10.(2j) 4-(1-(diphenylsilyl)vinyl)aniline - prepared from (4-ethynyl)aniline $(1.0 \mathrm{mmol})$ and diphenylsilane $(1.0 \mathrm{mmol})$ according to general procedure A. Purified by extraction with $n$ hexane followed by filtration through silica plug. Volatiles were removed under lower pressure affording pure product (brown oil, $300.9 \mathrm{mg}$ - >99\%).<smiles>C=C(c1ccc(N)cc1)[SiH](c1ccccc1)c1ccccc1</smiles>

${ }^{1} \mathrm{H}$ NMR $\left(300 \mathrm{MHz}, \mathrm{CDCl}_{3}\right): \delta 7.62-7.55(\mathrm{~m}, 4 \mathrm{H}), 7.42-7.32(\mathrm{~m}$, $6 \mathrm{H}), 7.25-7.19(\mathrm{~m}, 2 \mathrm{H}), 6.62-6.56(\mathrm{~m}, 2 \mathrm{H}), 6.25-6.22(\mathrm{dd}, 1 \mathrm{H}, \mathrm{J}$ $=2.3 \mathrm{~Hz}), 5.55-5.52(\mathrm{~d}, 1 \mathrm{H}, J=2.4 \mathrm{~Hz}), 5.39(\mathrm{~s}, 1 \mathrm{H}), 3.63(\mathrm{~s}, 2 \mathrm{H})$

${ }^{13} \mathrm{C}$ NMR $\left(75 \mathrm{MHz}, \mathrm{CDCl}_{3}\right): \delta 145.7,144.6,135.8,133.4,133.0$, $129.7,129.2,128.0,127.7,115.0$

Corresponds to previously reported data [3] 
2.11.(2k) (E)-oct-4-en-4-yldiphenylsilane - prepared from oct-4-yne $(1.0 \mathrm{mmol})$ and diphenylsilane $(1.0 \mathrm{mmol})$ according to general procedure B. Purified by extraction with $n$ hexane followed by filtration through silica plug. Volatiles were removed under lower pressure affording pure product (pale yellow oil, $280.3 \mathrm{mg}-95 \%$ ).<smiles>CCC/C=C(\CCC)[SiH](c1ccccc1)c1ccccc1</smiles>

${ }^{1} \mathrm{H}$ NMR $\left(300 \mathrm{MHz}, \mathrm{CDCl}_{3}\right): \delta 7.60-7.55(\mathrm{~m}, 4 \mathrm{H}), 7.42-7.35(\mathrm{~m}, 4 \mathrm{H})$, $5.96-5.90(\mathrm{t}, 1 \mathrm{H}), 6.28-6.25(\mathrm{~d}, 1 \mathrm{H}, J=1.8 \mathrm{~Hz}), 5.10(\mathrm{~s}, 1 \mathrm{H}), 2.26-2.14$ $(\mathrm{m}, 4 \mathrm{H}), 1.48-1.38(\mathrm{~h}, 2 \mathrm{H}), 1.38-1.28(\mathrm{~h}, 2 \mathrm{H}), 0.97-0.90(\mathrm{t}, 3 \mathrm{H}), 0.88$ $-0.81(\mathrm{t}, 3 \mathrm{H})$,

${ }^{13} \mathrm{C}$ NMR $\left(75 \mathrm{MHz}, \mathrm{CDCl}_{3}\right): \delta 146.7,135.7,135.1,134.3,129.4,127.9$, $32.6,30.9,23.1,22.6,14.3,14.0$

Corresponds to previously reported data [3]

2.12.(2l) Oct-1-en-2-yldiphenylsilane - prepared from okt-1-yne $(0.5 \mathrm{mmol})$ and diphenylsilane $(0.5 \mathrm{mmol})$ according to general procedure A. Purified by extraction with $n$-hexane followed by filtration through silica plug. Volatiles were removed under lower pressure affording pure product (pale yellow oil, $146.2 \mathrm{mg}->99 \%$ ).<smiles>C=C(CCCCCC)[SiH](c1ccccc1)c1ccccc1</smiles>

${ }^{1} \mathrm{H}$ NMR $\left(300 \mathrm{MHz}, \mathrm{CDCl}_{3}\right): \delta 7.61-7.51(\mathrm{~m}, 4 \mathrm{H}), 7.45-7.31(\mathrm{~m}, 6 \mathrm{H})$, $5.93-5.80(\mathrm{~b}, 1 \mathrm{H}), 5.50-5.43(\mathrm{~d}, 1 \mathrm{H}, J=2.8 \mathrm{~Hz}), 5.07(\mathrm{~s}, 1 \mathrm{H}), 2.28-$ $2.17(\mathrm{t}, 2 \mathrm{H}), 1.46-1.34(\mathrm{~m}, 2 \mathrm{H}), 1.27-1.17(\mathrm{~m}, 6 \mathrm{H}), 0.89-0.79(\mathrm{~m}$, $3 \mathrm{H})$

${ }^{13} \mathrm{C} \mathrm{NMR}\left(75 \mathrm{MHz}, \mathrm{CDCl}_{3}\right): \delta 146.6,135.7,133.4,129.6,129.5,128.0$, $37.0,31.7,29.0,28.8,22.6,14.1$

Corresponds to previously reported data [4]

2.13.(2m) ((3-(diphenylsilyl)but-3-en-1-yl)oxy)dimethyl(phenyl)silane - (but-3-yn-1-yloxy)-dimethyl(phenyl)silane $(0.5 \mathrm{mmol})$ and diphenylsilane $(0.5 \mathrm{mmol})$ according to general procedure A. Purified by extraction with $n$-hexane followed by filtration through silica plug. Volatiles were removed under lower pressure affording pure product (colorless oil, $140.1 \mathrm{mg}-$ $72 \%)$.<smiles>C=C(CCO[Si](C)(C)c1ccccc1)[SiH](c1ccccc1)c1ccccc1</smiles>

${ }^{1} \mathrm{H}$ NMR $\left(300 \mathrm{MHz}, \mathrm{CDCl}_{3}\right): \delta 7.58-7.50(\mathrm{~m}, 5 \mathrm{H}), 7.45-7.33$ $(\mathrm{m}, 10 \mathrm{H}), 5.99-5.83(\mathrm{~m}, 1 \mathrm{H}), 5.56(\mathrm{~s}, 1 \mathrm{H}), 5.09(\mathrm{~s}, 1 \mathrm{H}), 3.68-$ $3.58(\mathrm{t}, 2 \mathrm{H}), 2.55-2.45(\mathrm{t}, 1 \mathrm{H}), 0.35-0.26(\mathrm{~s}, 6 \mathrm{H})$

HRMS (ESI): calculated for $\left[\mathrm{C}_{24} \mathrm{H}_{28} \mathrm{Si}_{2} \mathrm{ONa}\right]^{+}$requires $411.1571 \mathrm{~m} / \mathrm{z}$, found $411.1575 \mathrm{~m} / \mathrm{z}$ 
2.14.(2n) Diphenyl(1-(p-tolyl)vinyl)silane - prepared from (4-methyl)phenylacetylene (0.5 $\mathrm{mmol})$ and diphenylsilane $(0.5 \mathrm{mmol})$ according to general procedure $\mathbf{B}$. Purified by extraction with $n$-hexane followed by filtration through silica plug. Volatiles were removed under lower pressure affording pure product (pale yellow oil, $142.8 \mathrm{mg}-95 \%$ ).<smiles>C=C(c1ccc(C)cc1)[SiH](c1ccccc1)c1ccccc1</smiles>

${ }^{1} \mathrm{H}$ NMR $\left(300 \mathrm{MHz}, \mathrm{CDCl}_{3}\right): \delta 7.59-7.53(\mathrm{~m}, 4 \mathrm{H}), 7.41-7.31(\mathrm{~m}, 7 \mathrm{H})$, $\delta 7.24-7.22(\mathrm{~m}, 1 \mathrm{H}), 7.08-7.03(\mathrm{~m}, 2 \mathrm{H}), 6.29-6.23(\mathrm{dd}, 1 \mathrm{H}, J=2.1$ $\mathrm{Hz}), 5.66-5.59(\mathrm{~d}, 1 \mathrm{H}, J=2.2 \mathrm{~Hz}), 5.37(\mathrm{~s}, 1 \mathrm{H}), 2.29(\mathrm{~s}, 3 \mathrm{H})$

${ }^{13} \mathrm{C}$ NMR $\left(75 \mathrm{MHz}, \mathrm{CDCl}_{3}\right): \delta 145.5,139.9,136.8,135.8,133.2,131.37$, $129.8,129.1,128.1,126.6,21.1$

Corresponds to previously reported data [3]

2.15.(20) Diphenyl(1-( $m$-tolyl)vinyl)silane - prepared from (3-methyl)phenylacetylene (1.0 $\mathrm{mmol})$ and diphenylsilane $(1.0 \mathrm{mmol})$ according to general procedure $\mathbf{A}$. Purified by extraction with $n$-hexane followed by filtration through silica plug. Volatiles were removed under lower pressure affording pure product (pale yellow oil, $281,3 \mathrm{mg}-94 \%$ ).<smiles>C=C(c1cccc(C)c1)[SiH](c1ccccc1)c1ccccc1</smiles>

${ }^{1} \mathrm{H}$ NMR $\left(300 \mathrm{MHz}, \mathrm{CDCl}_{3}\right): \delta 7.65-7.60(\mathrm{~m}, 4 \mathrm{H}), 7.48-7.38(\mathrm{~m}, 6 \mathrm{H})$, $\delta 7.23-7.17(\mathrm{~m}, 3 \mathrm{H}), 7.10-7.06(\mathrm{~m}, 1 \mathrm{H}), 6.33-6.31(\mathrm{dd}, 1 \mathrm{H}, J=2.5$ $\mathrm{Hz}), 5.73-5.71(\mathrm{~d}, 1 \mathrm{H}, \mathrm{J}=2.5 \mathrm{~Hz}), 4.74(\mathrm{~s}, 1 \mathrm{H}), 2.24(\mathrm{~s}, 3 \mathrm{H})$

${ }^{13} \mathrm{C}$ NMR $\left(75 \mathrm{MHz}, \mathrm{CDCl}_{3}\right): \delta 148.0,143.4,135.7,134.6,133.8,132.9$, $130.1,129.8,128.1,128.0,126.4,125.5,20.4$

Corresponds to previously reported data [3]

2.16.(2p) Diphenyl(1-(o-tolyl)vinyl)silane - prepared from (2-methyl)phenylacetylene (1.0 $\mathrm{mmol})$ and diphenylsilane $(1.0 \mathrm{mmol})$ according to general procedure $\mathbf{A}$. Purified by extraction with $n$-hexane followed by filtration through silica plug. Volatiles were removed under lower pressure affording pure product (pale yellow oil, 298,5 $\mathrm{mg}->99 \%$ ).<smiles>C=C(c1ccccc1C)[SiH](c1ccccc1)c1ccccc1</smiles>

${ }^{1} \mathrm{H}$ NMR $\left(300 \mathrm{MHz}, \mathrm{CDCl}_{3}\right): \delta 7.63-7.57(\mathrm{~m}, 4 \mathrm{H}), 7.48-7.37(\mathrm{~m}, 6 \mathrm{H})$, $\delta 7.19-7.06(\mathrm{~m}, 3 \mathrm{H}), 6.98-6.95(\mathrm{~d}, 1 \mathrm{H}), 6.01-5.98(\mathrm{~d}, 1 \mathrm{H}, J=3.1 \mathrm{~Hz})$, $5.95-5.93(\mathrm{~d}, 1 \mathrm{H}, J=3.1 \mathrm{~Hz}), 5.26(\mathrm{~s}, 1 \mathrm{H}), 2.24(\mathrm{~s}, 3 \mathrm{H})$

${ }^{13} \mathrm{C}$ NMR $\left(75 \mathrm{MHz}, \mathrm{CDCl}_{3}\right): \delta 148.0,143.4,135.7,134.5,133.3,132.9$, $130.1,129.8,128.0,128.0,126.4,125.4,20.4$

Corresponds to previously reported data [3] 
2.17.(2r) 4-(1-(diphenylsilyl)vinyl)benzonitrile - prepared from 4-ethynylbenzonitrile $(0.5 \mathrm{mmol})$ and diphenylsilane $(0.5 \mathrm{mmol})$ according to general procedure $\mathbf{B}$ at $40^{\circ} \mathrm{C}$. Purified by extraction with $n$-hexane followed by filtration through silica plug. Mixture required further purification by column chromatography on silica gel (hexane:ethyl acetate $=2: 1$ ). Volatiles were removed under lower pressure affording pure product (yellow oil, $85.3 \mathrm{mg}-55 \%$ ).<smiles>C=C(c1ccc(C#N)cc1)[SiH](c1ccccc1)c1ccccc1</smiles>

${ }^{1} \mathrm{H}$ NMR $\left(300 \mathrm{MHz}, \mathrm{CDCl}_{3}\right): \delta 7.57-7.51(\mathrm{~m}, 6 \mathrm{H}), 7.45-7.34(\mathrm{~m}$, $8 \mathrm{H}), 6.34-6.27(\mathrm{dd}, 1 \mathrm{H}, J=2.0,0.6 \mathrm{~Hz}), 5.84-5.79(\mathrm{~d}, 1 \mathrm{H}, J=2.1$ $\mathrm{Hz}), 5.40-5.33(\mathrm{~s}, 1 \mathrm{H})$

${ }^{13} \mathrm{C}$ NMR $\left(75 \mathrm{MHz}, \mathrm{CDCl}_{3}\right): \delta 147.9,145.3,135.7,134.5,132.2$, $132.0,130.2,128.2,127.4,118.9,110.6$

Corresponds to previously reported data [3]

2.18.(2s) Diphenyl(1-(thiophen-3-yl)vinyl)silane - prepared from 3-ethynylthiophene (0.5 $\mathrm{mmol})$ and diphenylsilane $(0.5 \mathrm{mmol})$ according to general procedure A. Purified by extraction with $n$-hexane followed by filtration through silica plug. Volatiles were removed under lower pressure affording pure product (brown oil, $144.2 \mathrm{mg}-98 \%$ ).<smiles>C=C(c1ccsc1)[SiH](c1ccccc1)c1ccccc1</smiles>

${ }^{1} \mathrm{H}$ NMR $\left(300 \mathrm{MHz}, \mathrm{CDCl}_{3}\right): \delta 7.63-7.58(\mathrm{~m}, 4 \mathrm{H}), 7.43-7.35(\mathrm{~m}, 6 \mathrm{H})$, $7.16-7.12(\mathrm{~m}, 1 \mathrm{H}), 6.96-6.91(\mathrm{~m}, 1 \mathrm{H}), 6.90-6.85(\mathrm{~m}, 1 \mathrm{H}), 6.35(\mathrm{~s}$, $1 \mathrm{H}), 5.52-5.47(\mathrm{~d}, 1 \mathrm{H}, J=1.6 \mathrm{~Hz}), 5.42(\mathrm{~s}, 1 \mathrm{H})$

${ }^{13} \mathrm{C}$ NMR $\left(75 \mathrm{MHz}, \mathrm{CDCl}_{3}\right): \delta 146.4,137.9,135.8,134.7,134.3,132.4$, $130.0,129.6,128.1,127.5,126.0,124.2$

Corresponds to previously reported data [3]

2.19.(2t) (E)-(1,2-diphenylvinyl)diphenylsilane - prepared from diphenylacetylene $(0.5 \mathrm{mmol})$ and diphenylsilane $(0.5 \mathrm{mmol})$ according to general procedure A. Purified by extraction with $n$ hexane followed by filtration through silica plug. Volatiles were removed under lower pressure affording pure product (yellow oil, $176.8 \mathrm{mg}-98 \%$ ).<smiles>C(=C(c1ccccc1)[SiH](c1ccccc1)c1ccccc1)c1ccccc1</smiles>

${ }^{1} \mathrm{H}$ NMR $\left(300 \mathrm{MHz}, \mathrm{CDCl}_{3}\right): \delta 7.60-7.54(\mathrm{~m}, 4 \mathrm{H}), 7.46-7.36(\mathrm{~m}, 6 \mathrm{H})$, $7.42-7.33(\mathrm{~m}, 6 \mathrm{H}), 7.24-7.15(\mathrm{~m}, 3 \mathrm{H}), 7.13-7.08(\mathrm{~m}, 3 \mathrm{H}), 7.04-6.98$ $(\mathrm{m}, 5 \mathrm{H}), 5.28(\mathrm{~s}, 1 \mathrm{H})$

${ }^{13} \mathrm{C}$ NMR $\left(75 \mathrm{MHz}, \mathrm{CDCl}_{3}\right): \delta 142.9,141.6,140.2,136.9,135.9,133.0$, $129.8,129.7,128.6,128.1,128.0,127.9,127.5,126.2$

Corresponds to previously reported data [3]

2.20.(2u) 3-(diphenylsilyl)but-3-en-1-yl benzoate - prepared from butyn-3-yl benzoate (0.5 $\mathrm{mmol})$ and diphenylsilane $(0.5 \mathrm{mmol})$ according to general procedure A. Purified by extraction with $n$-hexane followed by filtration through silica plug. Volatiles were removed under lower pressure affording pure product (colourless, $178.2 \mathrm{mg}-99 \%$ ).<smiles>C=C(CCOC(=O)c1ccccc1)[SiH](c1ccccc1)c1ccccc1</smiles>

${ }^{1} \mathrm{H}$ NMR $\left(300 \mathrm{MHz}, \mathrm{CDCl}_{3}\right): \delta 8.02-7.95(\mathrm{~m}, 2 \mathrm{H}), 7.61-7.51$ $(\mathrm{m}, 5 \mathrm{H}), 7.45-7.32(\mathrm{~m}, 8 \mathrm{H}), 6.07-6.01(\mathrm{~d}, 1 \mathrm{H}, J=1.4 \mathrm{~Hz})$, $5.65-5.57(\mathrm{~d}, 1 \mathrm{H}, J=2.3 \mathrm{~Hz}), 5.15(\mathrm{~s}, 1 \mathrm{H}), 4.43-4.31(\mathrm{t}, 2 \mathrm{H})$, $2.76-2.67(\mathrm{t}, 2 \mathrm{H})$

${ }^{13} \mathrm{C}$ NMR $\left(75 \mathrm{MHz}, \mathrm{CDCl}_{3}\right): \delta 166.4,142.2,135.6,132.8,132.6$, $132.5,130.3,129.9,129.6,128.3,128.1,63.8,35.8$

HRMS (ESI): calculated for $\left[\mathrm{C}_{23} \mathrm{H}_{22} \mathrm{SiO}_{2} \mathrm{Na}\right]^{+}$requires $381.1281 \mathrm{~m} / \mathrm{z}$, found $381.1289 \mathrm{~m} / \mathrm{z}$ 
2.21. (but-3-yn-1-yloxy)dimethyl(phenyl)silane - But-3-yn-1-ol was silylated with dimethylphenylsilane according to reported procedure[6]. (but-3-yn-1yloxy)dimethyl(phenyl)silane was purified by extraction with $n$-hexane followed by filtration through silica plug. Volatiles were removed under reduced pressure.

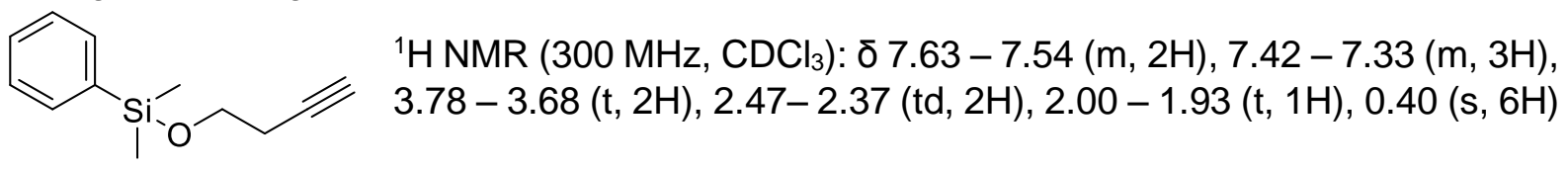

2.22.(3a) Phenyl(1-phenylvinyl)silane- prepared from phenylsilane $(1.0 \mathrm{mmol})$ and phenylacetylene $(1.0 \mathrm{mmol})$ according to general procedure $\mathbf{C}$. Purified by extraction with $n$ hexane followed by filtration through silica plug. Volatiles were removed under lower pressure affording pure product (yellow oil, $206.3 \mathrm{mg}-98 \%$ ).<smiles>C=C([SiH]c1ccccc1)c1ccccc1</smiles>

${ }^{1} \mathrm{H}$ NMR $\left(300 \mathrm{MHz}, \mathrm{CDCl}_{3}\right): \delta 7.58-7.51(\mathrm{~m}, 2 \mathrm{H}), 7.37-7.27(\mathrm{~m}, 6 \mathrm{H})$, $7.25-7.17(\mathrm{~m}, 2 \mathrm{H}), 6.23-6.18(\mathrm{~d}, 1 \mathrm{H}, J=2.4 \mathrm{~Hz}), 5.81-5.77(\mathrm{~d}, 1 \mathrm{H}, J$ $=2.3 \mathrm{~Hz}), 4.80(\mathrm{~s}, 2 \mathrm{H})$

${ }^{13} \mathrm{C} \mathrm{NMR}\left(75 \mathrm{MHz}, \mathrm{CDCl}_{3}\right): \delta 144.2,142.3,135.6,131.4,131.2,129.9$, 128.6, 128.2, 127.3, 126.5

Corresponds to previously reported data [7]

2.23.(3g) Phenyl(1-( $m$-tolyl)vinyl)silane - prepared from phenylsilane $(1.0 \mathrm{mmol})$ and 3 ethynyltoluene $(1.0 \mathrm{mmol}$ ) according to general procedure C. Purified by extraction with $n$ hexane followed by filtration through silica plug. Volatiles were removed under lower pressure affording pure product (yellow oil, $210.3 \mathrm{mg}-94 \%$ ).<smiles>C=C(c1cccc(C)c1)[SiH](C)c1ccccc1</smiles>

${ }^{1} \mathrm{H}$ NMR $\left(300 \mathrm{MHz}, \mathrm{CDCl}_{3}\right): \delta 7.65-7.58(\mathrm{~m}, 2 \mathrm{H}), 7.46-7.34(\mathrm{~m}, 3 \mathrm{H})$, $7.25-7.16(\mathrm{~m}, 3 \mathrm{H}), 7.12-7.04(\mathrm{~m}, 1 \mathrm{H}), 6.27-6.23(\mathrm{~d}, 1 \mathrm{H}, J=2.5 \mathrm{~Hz})$, $5.87-5.79(\mathrm{~d}, 1 \mathrm{H}, J=2.4 \mathrm{~Hz}), 4.86(\mathrm{~s}, 2 \mathrm{H}), 2.40-2.34(\mathrm{~s}, 3 \mathrm{H})$

${ }^{13} \mathrm{C}$ NMR $\left(75 \mathrm{MHz}, \mathrm{CDCl}_{3}\right): \delta 144.3,142.3,138.1,135.6,131.1,129.9$, $128.4,128.1,127.1,123.7,21.5$

Corresponds to previously reported data [7]

2.24.(3h) Phenyl(1-(o-tolyl)vinyl)silane - prepared from phenylsilane $(1.0 \mathrm{mmol})$ and 2ethynyltoluene $(1.0 \mathrm{mmol})$ according to general procedure $\mathbf{C}$. Purified by extraction with $n$ hexane followed by filtration through silica plug. Volatiles were removed under lower pressure affording pure product (yellow oil, $223.9 \mathrm{mg}-99 \%$ ).<smiles>C=C([SiH]c1ccccc1)c1ccccc1C</smiles>

${ }^{1} \mathrm{H}$ NMR $\left(300 \mathrm{MHz}, \mathrm{CDCl}_{3}\right): \delta 7.62-7.53(\mathrm{~m}, 2 \mathrm{H}), 7.44-7.32(\mathrm{~m}, 3 \mathrm{H})$, $7.19-7.08(\mathrm{~m}, 3 \mathrm{H}), 7.02-6.89(\mathrm{~m}, 1 \mathrm{H}), 5.95-5.91(\mathrm{~d}, 1 \mathrm{H}, J=2.0 \mathrm{~Hz})$, $5.90-5.86(\mathrm{~d}, 1 \mathrm{H}, J=1.9 \mathrm{~Hz}), 4.71(\mathrm{~s}, 2 \mathrm{H}), 2.25(\mathrm{~s}, 3 \mathrm{H})$

${ }^{13} \mathrm{C} \mathrm{NMR}\left(75 \mathrm{MHz}, \mathrm{CDCl}_{3}\right): \delta 146.5,143.1,135.6,133.0,130.1,129.9$, $128.0,127.9,126.6,125.6,20.3$

Corresponds to previously reported data [7] 
2.25.(4a) Dimethyl(phenyl)(1-phenylvinyl)silane - prepared from dimethyl(phenyl)silane (1.0 $\mathrm{mmol})$ and phenylacetylene $(1.0 \mathrm{mmol})$ according to general procedure $\mathbf{B}$. Purified by extraction with $n$-hexane followed by filtration through silica plug. Volatiles were removed under lower pressure affording pure product (yellow oil, $225.0 \mathrm{mg}-94 \%$ ).<smiles>C=C(c1ccccc1)[Si](C)(C)c1ccccc1</smiles>

${ }^{1} \mathrm{H}$ NMR $\left(300 \mathrm{MHz}, \mathrm{CDCl}_{3}\right): \delta 7.62-7.51(\mathrm{~m}, 2 \mathrm{H}), 7.41-7.32(\mathrm{~m}, 3 \mathrm{H})$, $7.25-7.17(\mathrm{~m}, 3 \mathrm{H}), 7.12(\mathrm{~m}, 2 \mathrm{H}), 6.04-5.95(\mathrm{~d}, 1 \mathrm{H}, J=2.8 \mathrm{~Hz}), 5.72-$ $5.63(\mathrm{~d}, 1 \mathrm{H}, \mathrm{J}=2.9 \mathrm{~Hz}), 0.41(\mathrm{~s}, 6 \mathrm{H})$

${ }^{13} \mathrm{C}$ NMR $\left(75 \mathrm{MHz}, \mathrm{CDCl}_{3}\right): \delta 153.3,146.5,140.6,136.4,131.5,131.4$, $130.4,130.1,129.2,128.7,0.0$

Corresponds to previously reported data [8]

2.26.(4b) (1-(4-(tert-butyl)phenyl)vinyl)dimethyl(phenyl)silane - prepared from dimethyl(phenyl)silane $(1.0 \mathrm{mmol})$ and 1-(tert-butyl)-4-ethynylbenzene $(1.0 \mathrm{mmol})$ according to general procedure B. Purified by extraction with $n$-hexane followed by filtration through silica plug. Volatiles were removed under lower pressure affording pure product (yellow oil, 291.5

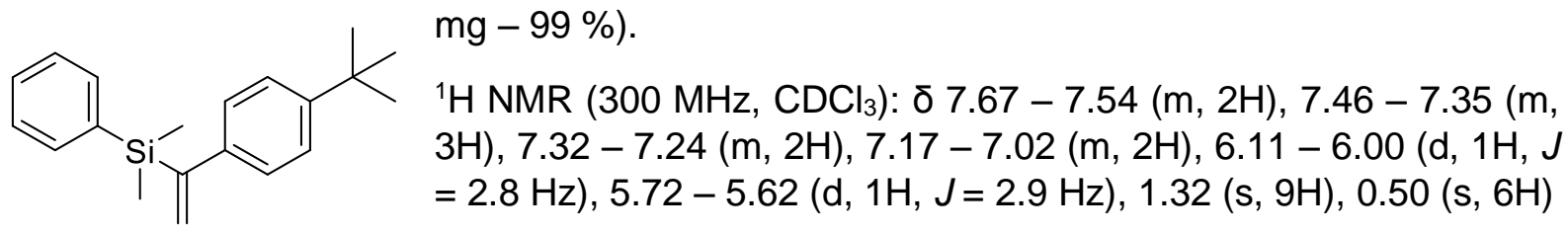

${ }^{13} \mathrm{C} \mathrm{NMR}\left(75 \mathrm{MHz}, \mathrm{CDCl}_{3}\right): \delta 152.3,151.5,143.0,140.8,136.2,131.2,130.8,130.0,128.6$, $127.2,36.6,33.5,0.0$

Corresponds to previously reported data [10]

2.27.(4c) (1-(4-bromophenyl)vinyl)dimethyl(phenyl)silane - prepared from dimethyl(phenyl)silane $(1.0 \mathrm{mmol})$ and 1-bromo-4-ethynylbenzene $(1.0 \mathrm{mmol})$ according to general procedure B. Purified by extraction with $n$-hexane followed by filtration through silica plug. Volatiles were removed under lower pressure affording pure product (yellow oil, 251.7 $\mathrm{mg}-79 \%)$.<smiles>C=C(c1ccc(Br)cc1)[Si](C)(C)c1ccccc1</smiles>

${ }^{1} \mathrm{H}$ NMR $\left(300 \mathrm{MHz}, \mathrm{CDCl}_{3}\right): \delta 7.59-7.49(\mathrm{~m}, 2 \mathrm{H}), 7.41-7.29(\mathrm{~m}$, $5 \mathrm{H}), 7.01-6.96(\mathrm{~m}, 2 \mathrm{H}), 6.01-5.92(\mathrm{~d}, 1 \mathrm{H}, J=2.7,0.6 \mathrm{~Hz}), 5.74-$ $5.66(\mathrm{~d}, 1 \mathrm{H}, J=2.8 \mathrm{~Hz}), 0.40(\mathrm{~s}, 6 \mathrm{H})$

${ }^{13} \mathrm{C} \mathrm{NMR}\left(75 \mathrm{MHz}, \mathrm{CDCl}_{3}\right): \delta 152.6,145.6,140.3,136.5,133.7,132.0$, $131.7,131.0,130.4,122.9,0.0$

Corresponds to previously reported data [8] 
2.28.(4d) (1-(4-methoxyphenyl)vinyl)dimethyl(phenyl)silane - prepared from dimethyl(phenyl)silane $(1.0 \mathrm{mmol})$ and 4-ethynylanisole $(1.0 \mathrm{mmol})$ according to general procedure B. Purified by extraction with $n$-hexane followed by filtration through silica plug. Volatiles were removed under lower pressure affording pure product (pale yellow oil, $251.7 \mathrm{mg}$ $-94 \%)$.

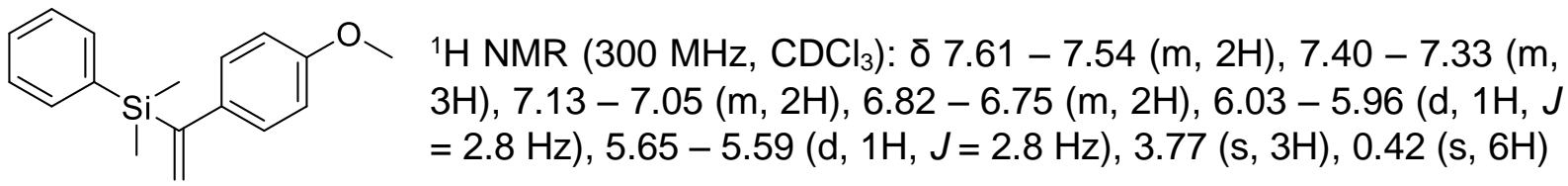

${ }^{13} \mathrm{C}$ NMR $\left(75 \mathrm{MHz}, \mathrm{CDCl}_{3}\right): \delta 160.6,152.1,140.7,138.6,136.2,131.2,130.2,130.1,130.0$, $115.7,57.4,0.0$

Corresponds to previously reported data [8]

2.29.(4e) 4-(1-(dimethyl(phenyl)silyl)vinyl)aniline - prepared form dimethyl(phenyl)silane (1.0 $\mathrm{mmol}$ ) and 4-ethynylaniline $(1.0 \mathrm{mmol})$ according to general procedure $\mathbf{B}$. Purified by extraction with $n$-hexane followed by filtration through silica plug. Volatiles were removed under lower pressure affording pure product (pale yellow oil, $189.4 \mathrm{mg}-75 \%$ ).<smiles>C=C(c1ccc(N)cc1)[Si](C)(C)c1ccccc1</smiles>

${ }^{1} \mathrm{H}$ NMR $\left(300 \mathrm{MHz}, \mathrm{CDCl}_{3}\right): \delta 7.63-7.52(\mathrm{~m}, 2 \mathrm{H}), 7.41-7.32(\mathrm{~m}$, $3 \mathrm{H}), 7.02-6.96(\mathrm{~m}, 2 \mathrm{H}), 5.59-5.54(\mathrm{~m}, 2 \mathrm{H}), 6.00-5.96(\mathrm{~d}, 1 \mathrm{H}, J$ $=2.8 \mathrm{~Hz}), 5.58-5.55(\mathrm{~d}, 1 \mathrm{H}, J=2.8 \mathrm{~Hz}), 3.78-3.45(\mathrm{~b}, 2 \mathrm{H}), 0.42$ $(\mathrm{s}, 6 \mathrm{H})$

${ }^{13} \mathrm{C}$ NMR $\left(75 \mathrm{MHz}, \mathrm{CDCl}_{3}\right): \delta 151.8,147.1,140.9,136.3,136.1$, $131.0,129.9,129.9,129.0,117.0,0.0$

HRMS (ESI): calculated for [ $\left.\mathrm{C}_{16} \mathrm{H}_{20} \mathrm{SiN}\right]^{+}$requires $254.1360 \mathrm{~m} / \mathrm{z}$, found $254.1365 \mathrm{~m} / \mathrm{z}$

2.30.(4f) dimethyl(phenyl)(1-(p-tolyl)vinyl)silane - prepared from dimethyl(phenyl)silane (1.0 $\mathrm{mmol})$ and 4-ethynyltoluene $(1.0 \mathrm{mmol})$ according to general procedure $\mathbf{B}$. Purified by extraction with $n$-hexane followed by filtration through silica plug. Volatiles were removed under lower pressure affording pure product (yellow oil, $243.4 \mathrm{mg}-96 \%$ ).<smiles>C=C(c1ccc(C)cc1)[Si](C)(C)c1ccccc1</smiles>

${ }^{1} \mathrm{H}$ NMR $\left(300 \mathrm{MHz}, \mathrm{CDCl}_{3}\right): \delta 7.63-7.53(\mathrm{~m}, 2 \mathrm{H}), 7.43-7.32(\mathrm{~m}, 3 \mathrm{H})$, $7.10-6.99(\mathrm{~m}, 4 \mathrm{H}), 6.05-5.95(\mathrm{~d}, 1 \mathrm{H}, J=2.8 \mathrm{~Hz}), 5.71-5.61(\mathrm{~d}, 1 \mathrm{H}$, $J=2.9 \mathrm{~Hz}), 2.31(\mathrm{~s}, 3 \mathrm{H}), 0.44(\mathrm{~s}, 6 \mathrm{H})$

${ }^{13} \mathrm{C} \mathrm{NMR}\left(75 \mathrm{MHz}, \mathrm{CDCl}_{3}\right): \delta 152.8,143.4,140.7,138.3,136.3,131.3$, $131.1,130.9,130.0,129.0,23.3,0.0$

Corresponds to previously reported data [9] 
2.31.(4g) dimethyl(phenyl)(1-( $m$-tolyl)vinyl)silane - prepared from dimethyl(phenyl)silane (1.0 $\mathrm{mmol}$ ) and 3-ethynyltoluene $(1.0 \mathrm{mmol})$ according to general procedure $\mathbf{B}$. Purified by extraction with $n$-hexane followed by filtration through silica plug. Volatiles were removed under lower pressure affording pure product (yellow oil, $231.3 \mathrm{mg}-92 \%$ ).

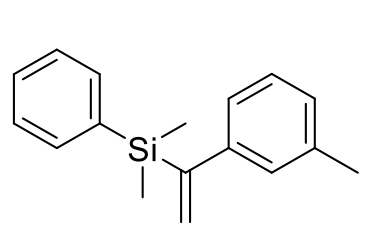

${ }^{1} \mathrm{H}$ NMR $\left(300 \mathrm{MHz}, \mathrm{CDCl}_{3}\right): \delta 7.61-7.53(\mathrm{~m}, 2 \mathrm{H}), 7.24-7.17(\mathrm{~m}, 3 \mathrm{H})$, $7.10-6.99(\mathrm{~m}, 2 \mathrm{H}), 6.91-6.84(\mathrm{~m}, 2 \mathrm{H}), 6.02-6.98(\mathrm{~d}, 1 \mathrm{H}, J=3.0$ $\mathrm{Hz}), 6.67-5.63(\mathrm{~d}, 1 \mathrm{H}, J=3.0 \mathrm{~Hz}), 2.07(\mathrm{~s}, 3 \mathrm{H}), 0.39(\mathrm{~s}, 6 \mathrm{H})$

${ }^{13} \mathrm{C}$ NMR $\left(75 \mathrm{MHz}, \mathrm{CDCl}_{3}\right): \delta 153.3,146.4,140.6,139.9,136.4,131.3$, $130.2,130.1,129.9,129.4,126.3,23.8,0.00$

HRMS (ESI): calculated for $\left[\mathrm{C}_{17} \mathrm{H}_{20} \mathrm{SiNa}\right]^{+}$requires $275.1226 \mathrm{~m} / \mathrm{z}$, found $275.1226 \mathrm{~m} / \mathrm{z}$

2.32.(4h) dimethyl(phenyl)(1-(o-tolyl)vinyl)silane - prepared from dimethyl(phenyl)silane (1.0 $\mathrm{mmol})$ and 2-ethynyltoluene $(1.0 \mathrm{mmol})$ according to general procedure B. Purified by extraction with $n$-hexane followed by filtration through silica plug. Volatiles were removed under lower pressure affording pure product (orange oil, $243.3 \mathrm{mg}-96 \%$ ).<smiles>C=C(c1ccccc1C)[Si](C)(C)c1ccccc1</smiles>

${ }^{1} \mathrm{H}$ NMR $\left(300 \mathrm{MHz}, \mathrm{CDCl}_{3}\right): \delta 7.52-7.44(\mathrm{~m}, 2 \mathrm{H}), 7.24-7.18(\mathrm{~m}, 3 \mathrm{H})$, $7.08-6.99(\mathrm{~m}, 3 \mathrm{H}), 6.94-6.88(\mathrm{~m}, 1 \mathrm{H}), 5.72-5.69(\mathrm{~d}, 1 \mathrm{H}, J=3.4 \mathrm{~Hz})$, $5.67-5.63(\mathrm{~d}, 1 \mathrm{H}, J=3.4 \mathrm{~Hz}), 2.06(\mathrm{~s}, 3 \mathrm{H}), 0.32(\mathrm{~s}, 6 \mathrm{H})$

${ }^{13} \mathrm{C}$ NMR $\left(75 \mathrm{MHz}, \mathrm{CDCl}_{3}\right): \delta 152.3,143.9,137.7,134.3,134.1,130.0$, $129.6,129.1,127.7,127.7,125.9,125.0,20.2,-3.0$

Corresponds to previously reported data [11]

2.33.(4i) (1-cyclopropylvinyl)dimethyl(phenyl)silane - prepared from dimethyl(phenyl)silane $(1.0 \mathrm{mmol})$ and cyclopropylacetylene $(1.0 \mathrm{mmol})$ according to general procedure B. Purified by extraction with $n$-hexane followed by filtration through silica plug. Volatiles were removed under lower pressure affording pure product (pale yellow oil, $193.1 \mathrm{mg}-95 \%$ ).

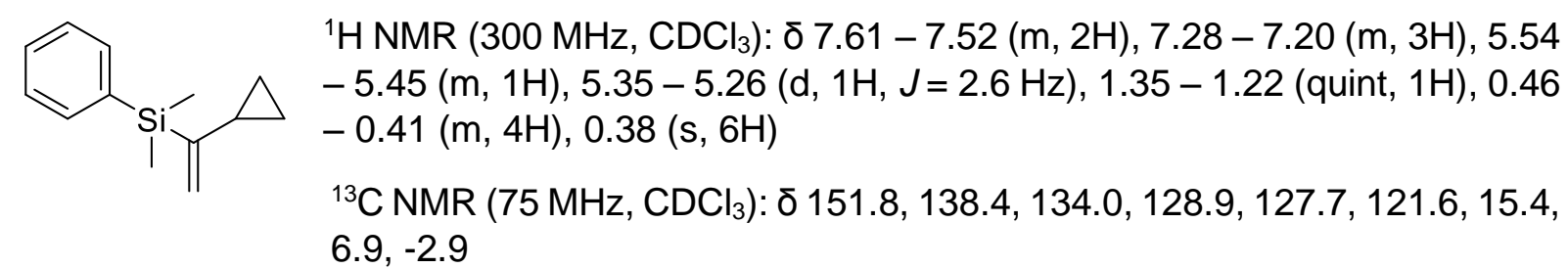

Corresponds to previously reported data [12] 


\section{Deuterium labelled experiment}
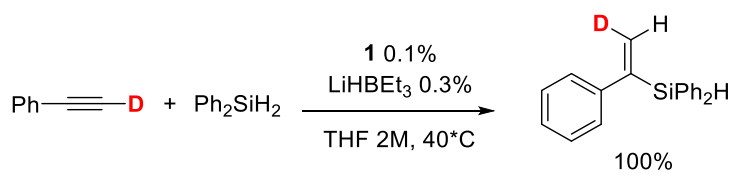

3.1 Hydrosililation of $D_{1}$-phenylaceylene with diphenylsilane.

To a flame-dried Schlenk bomb flask charged with argon, precatalyst (1 mol \%), THF (1.0 mL), diphenylsilane $(0.5 \mathrm{mmol})$, and $\mathrm{D}_{1}$-phenylacetylene $(0.5 \mathrm{mmol})$ were placed. Mixture was then heated to $40^{\circ} \mathrm{C}$ with stirring. After 10 minutes $\mathrm{LiHBEt}_{3}$ was added $(0.3 \mathrm{~mol} \%)$, and reaction vessel was then closed. After 1 hour, hexane was added to reaction mixture. Solution was then filtered through silica plug, and concentrated on rotavapor. The crude mixture was purified by evaporating volatiles on vacuum line followed by extraction with hexane. Regioselectivity was monitored by ${ }^{1} \mathrm{H}$ NMR.<smiles>[2H]C=C(c1ccccc1)[SiH](c1ccccc1)c1ccccc1</smiles>

${ }^{1} \mathrm{H}$ NMR $\left(300 \mathrm{MHz}, \mathrm{CDCl}_{3}\right): \delta 7.52-7.46(\mathrm{~m}, 4 \mathrm{H}), 7.36-7.24(\mathrm{~m}, 8 \mathrm{H})$, 7.21-7.11 (m, 3H), [6.23 - 6.19 Not observed], $5.63-5.56(\mathrm{~s}, 1 \mathrm{H}),$, $-5.28(\mathrm{~s}, 1 \mathrm{H})$ 
2a $\left(300 \mathrm{MHz}, \mathrm{CDCl}_{3}\right)$ :<smiles>C=C(c1ccccc1)[SiH](c1ccccc1)c1ccccc1</smiles>
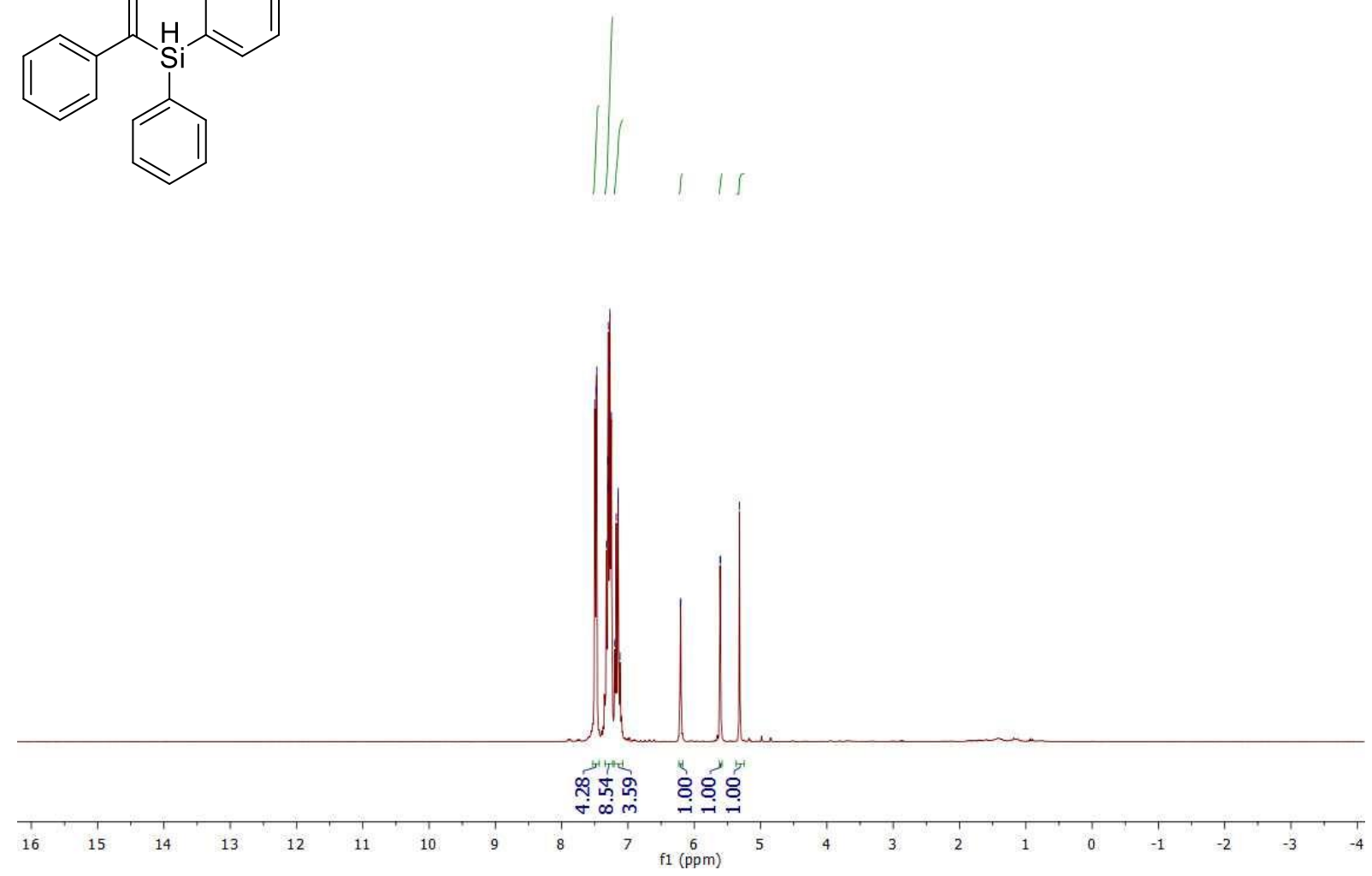

2a $\left(75 \mathrm{MHz}, \mathrm{CDCl}_{3}\right): \quad$ :<smiles>C=C(c1ccccc1)[SiH](c1ccccc1)c1ccccc1</smiles>

$\begin{array}{llllllllllllllllllllllllllllllllll}600 & 250 & 240 & 230 & 220 & 210 & 200 & 190 & 180 & 170 & 160 & 150 & 140 & 130 & 120 & 110 & 100 & 90 & 80 & 70 & 60 & 50 & 40 & 30 & 20 & 10 & 0 & -10 & -20 & -30 & -40 & -50 & -66\end{array}$ 
<smiles>C=C(c1ccc(C(C)(C)C)cc1)[Si](c1ccccc1)c1ccccc1</smiles>

2b $\left(300 \mathrm{MHz}, \mathrm{CDCl}_{3}\right)$ :

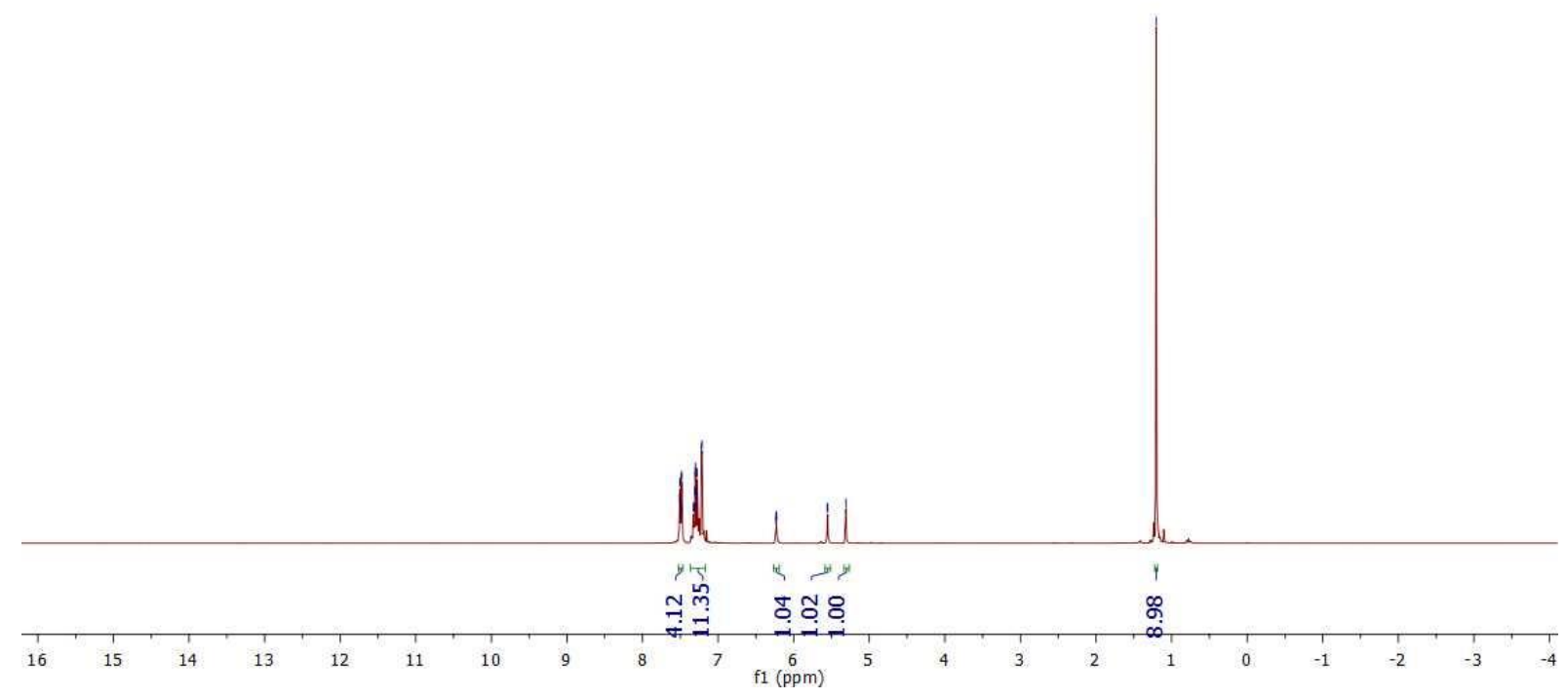

2b $\left(75 \mathrm{MHz}, \mathrm{CDCl}_{3}\right): \quad$ :<smiles>C=C(c1ccc(C(C)(C)C)cc1)[SiH](c1ccccc1)c1ccccc1</smiles> 
$2 c\left(300 \mathrm{MHz}, \mathrm{CDCl}_{3}\right):$ :<smiles>C=C(c1ccc(Br)cc1)[SiH](c1ccccc1)c1ccccc1</smiles>
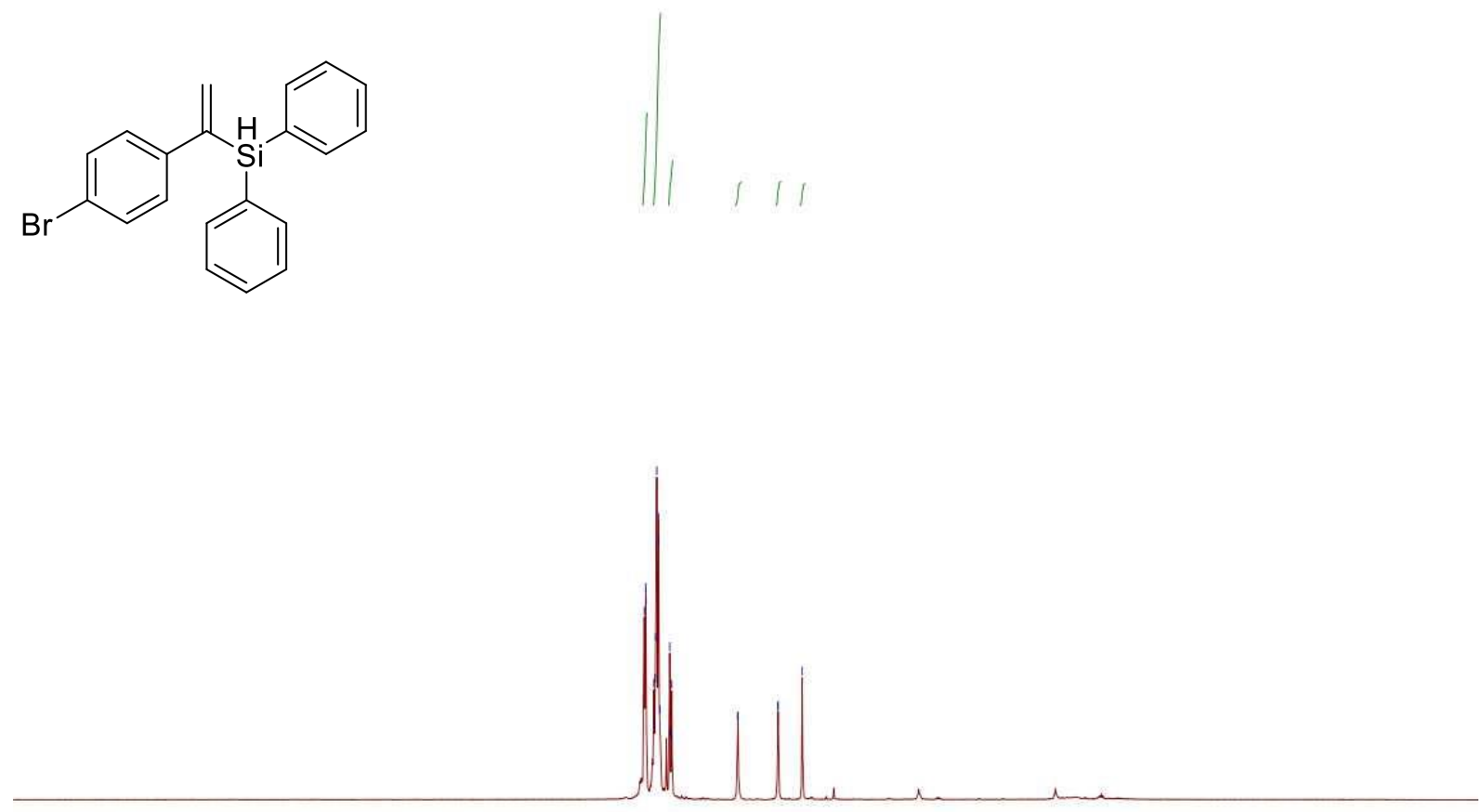

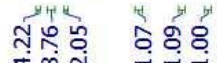

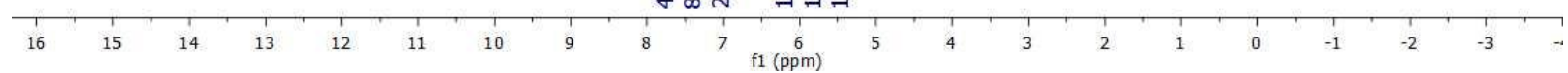

2c $\left(75 \mathrm{MHz}, \mathrm{CDCl}_{3}\right)$ :

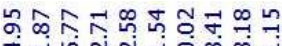

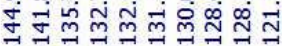<smiles>C=C(c1ccc(Br)cc1)[SiH](c1ccccc1)c1ccccc1</smiles>

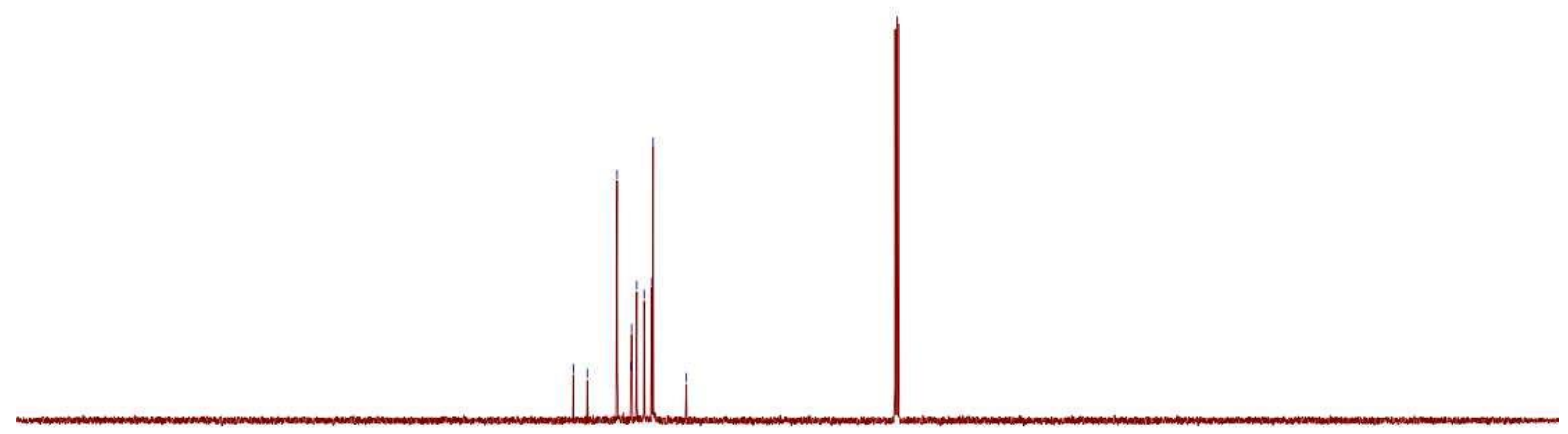

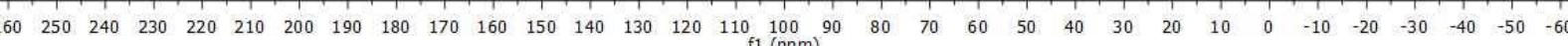




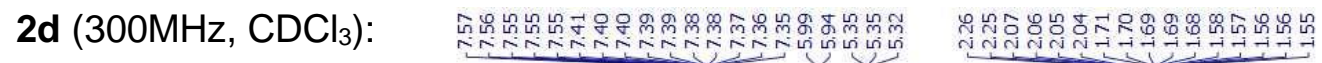<smiles>C=C(C1=CCCCC1)[SiH](c1ccccc1)c1ccccc1</smiles>
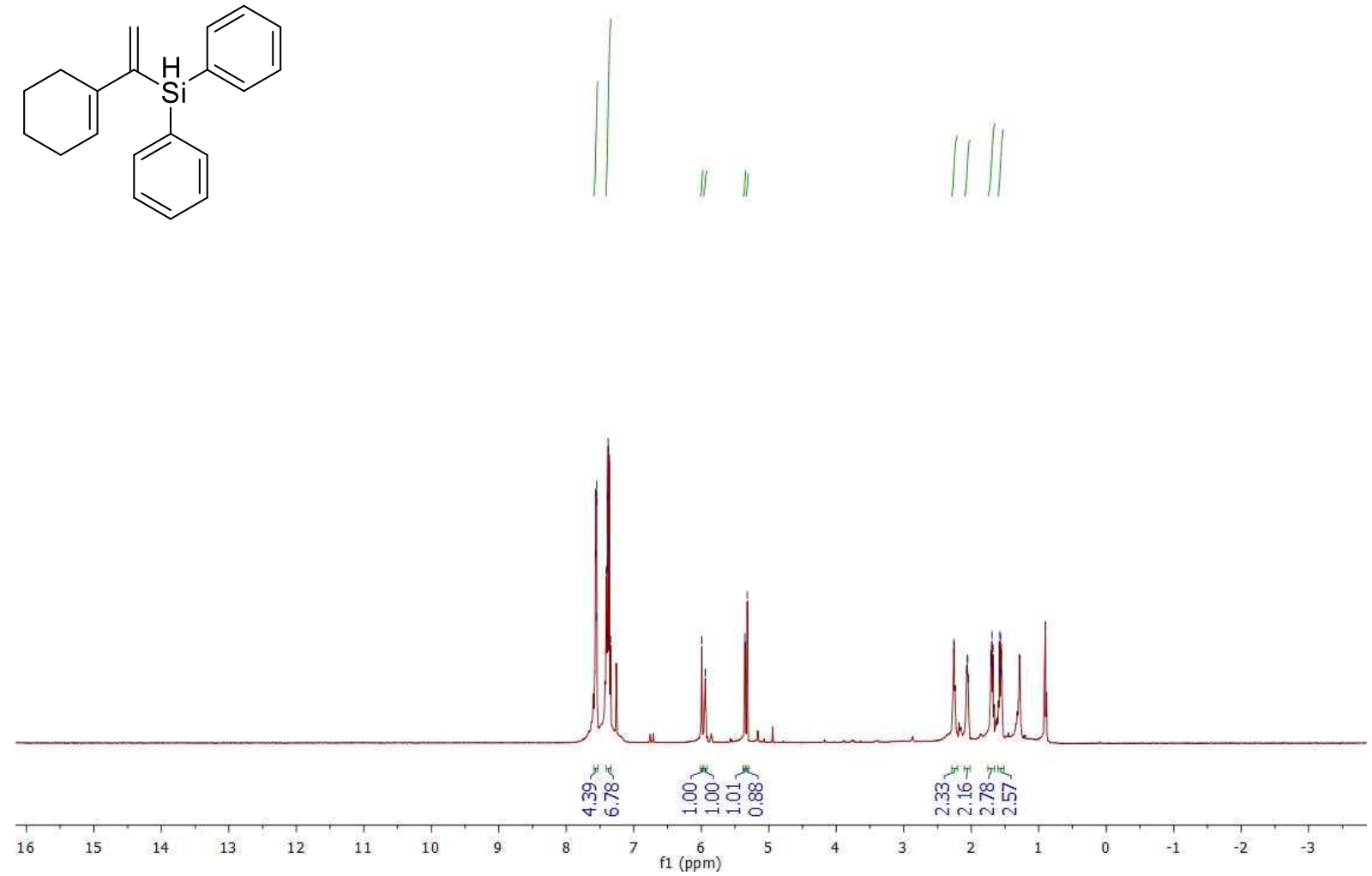

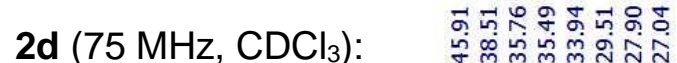

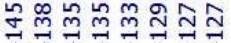

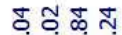

ำㅈำ<smiles>C=C(C1=CCCCC1)[SiH](c1ccccc1)c1ccccc1</smiles>

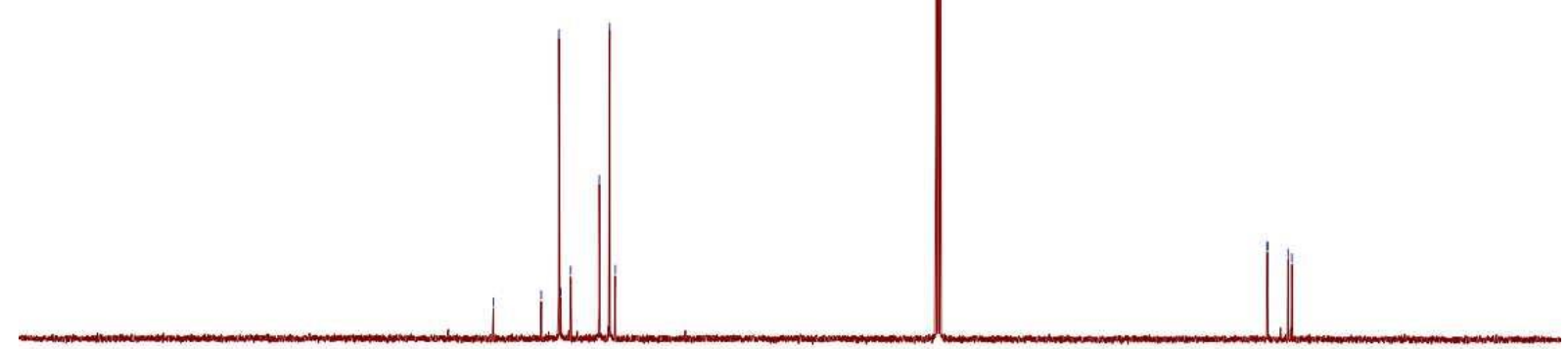

$\begin{array}{llllllllllllllllllllllllllllllllll}210 & 200 & 190 & 180 & 170 & 160 & 150 & 140 & 130 & 120 & 110 & 100 & 90 & 80 & 70 & 60 & 50 & 40 & 30 & 20 & 10 & 0 & -10\end{array}$ 
2e $\left(300 \mathrm{MHz}, \mathrm{CDCl}_{3}\right)$ :<smiles>C=C(Cc1ccccc1)[SiH](c1ccccc1)c1ccccc1</smiles>
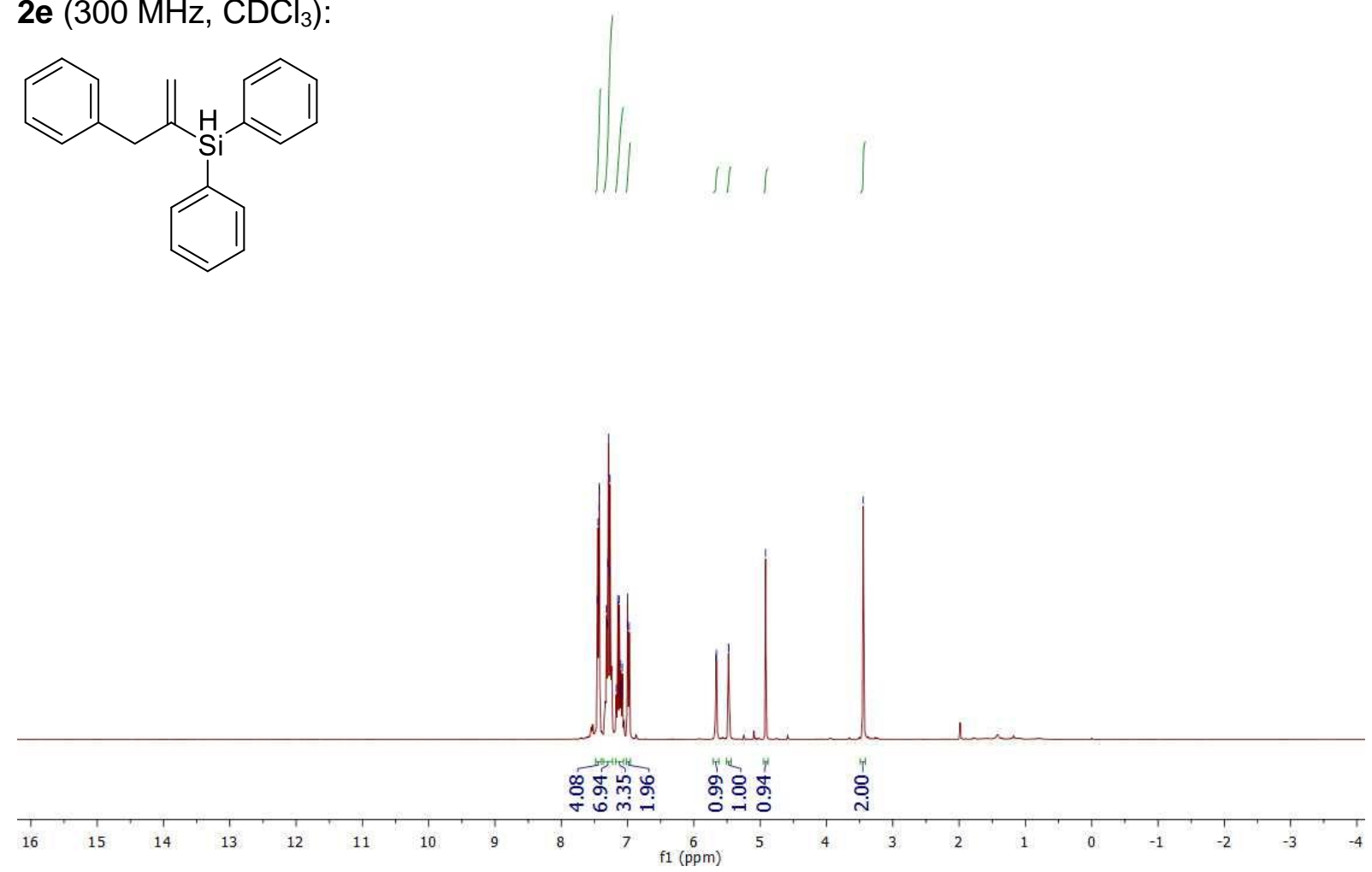<smiles>C=C(Cc1ccccc1)[Si](c1ccccc1)c1ccccc1</smiles> 


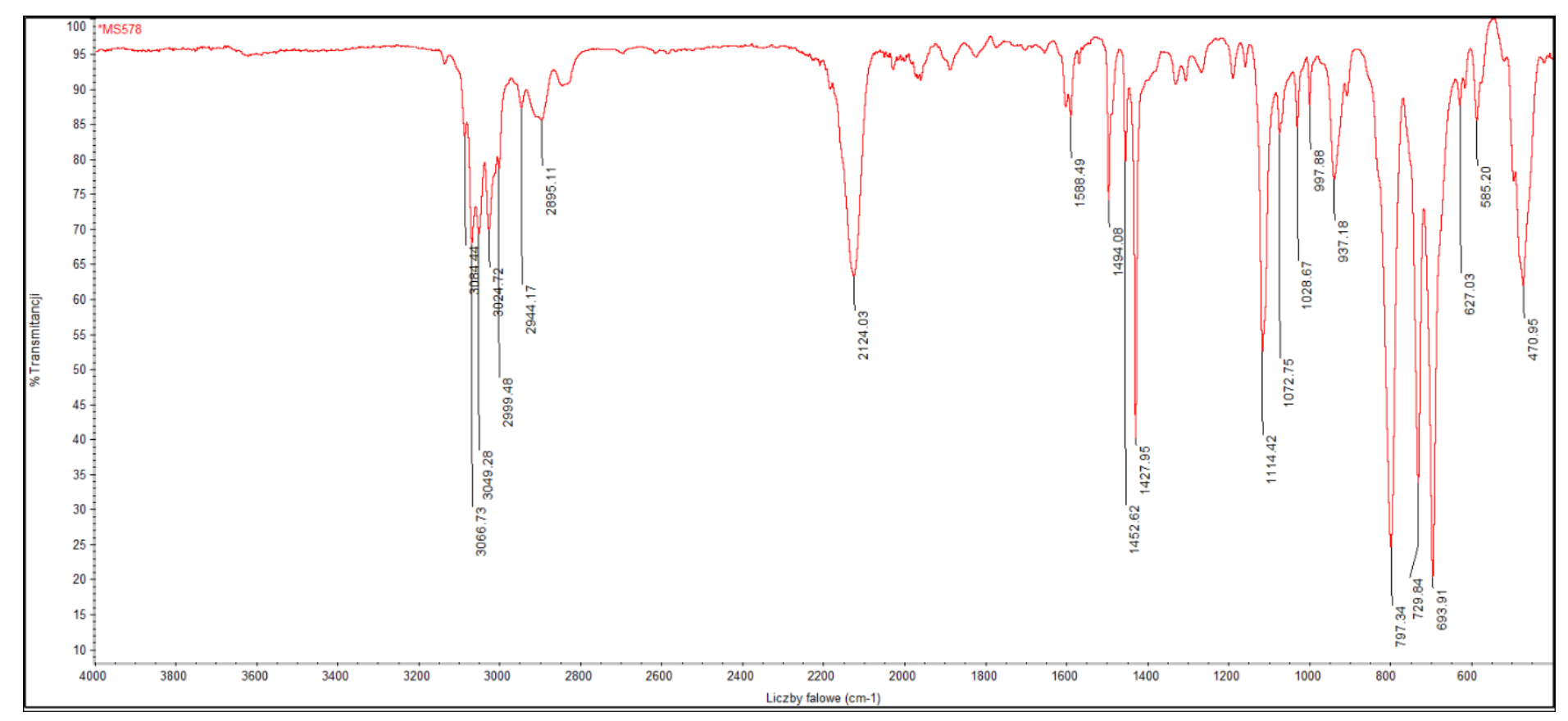




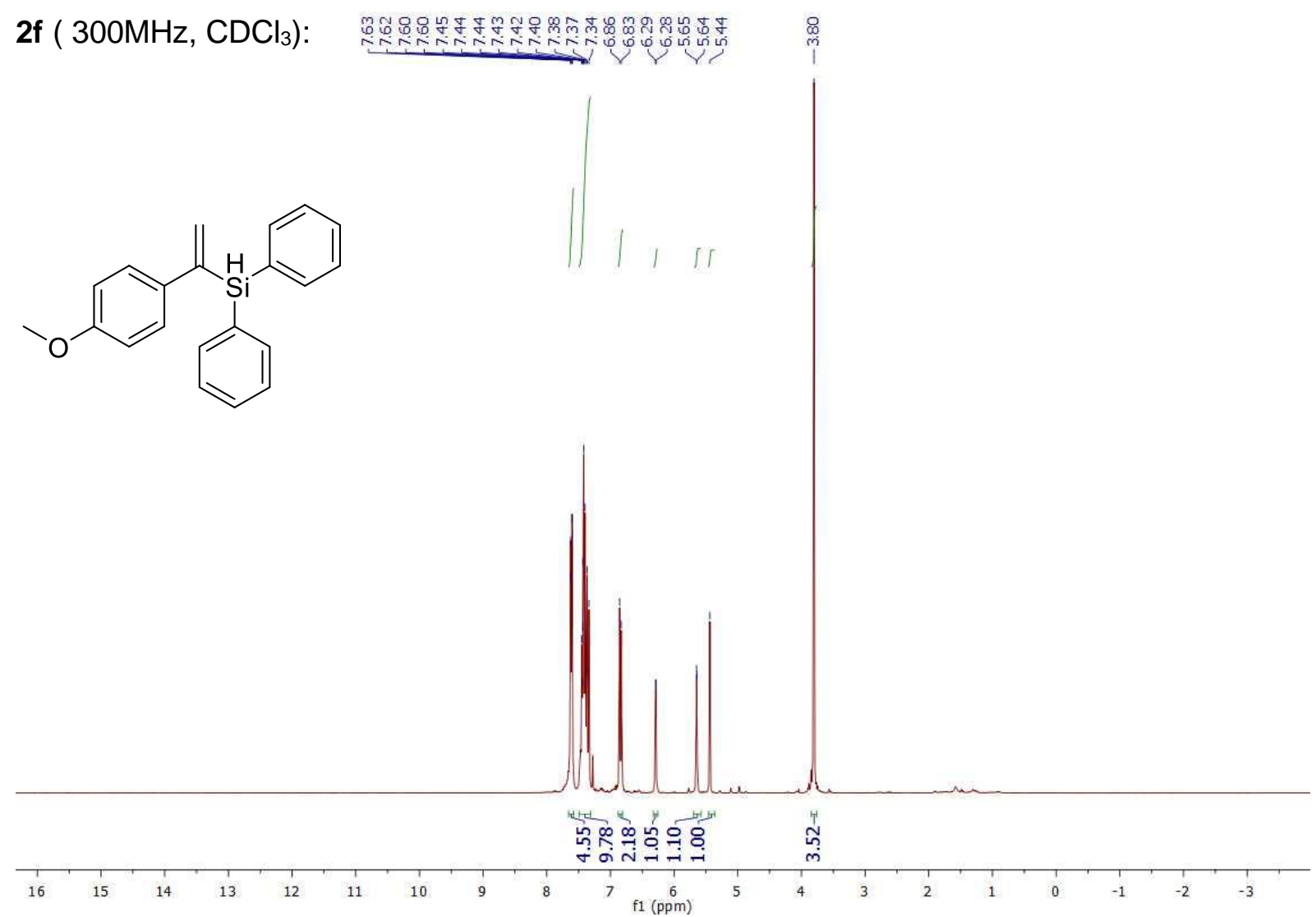

$2 f\left(75 \mathrm{MHz}, \mathrm{CDCl}_{3}\right):$

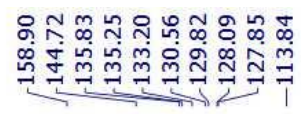

సึำ

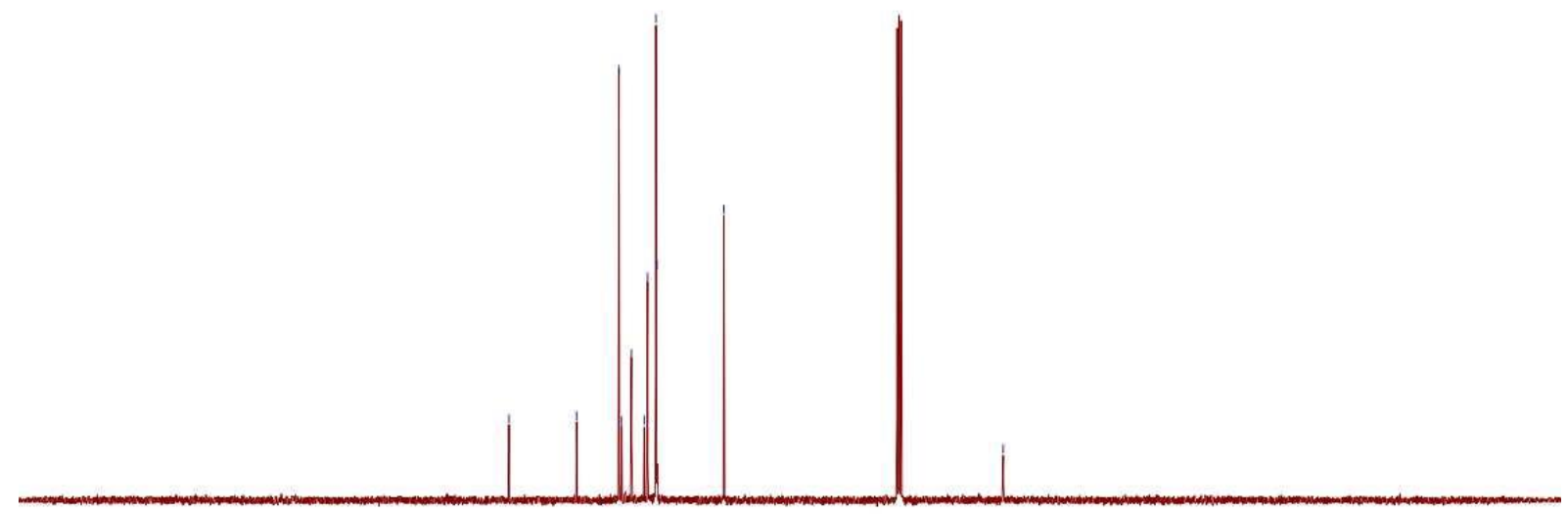

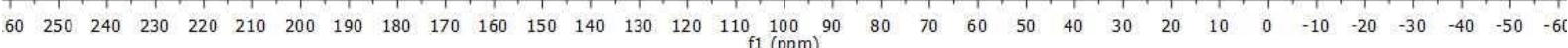




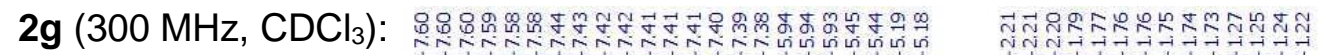<smiles>C=C(C1CCCCC1)[SiH](c1ccccc1)c1ccccc1</smiles>
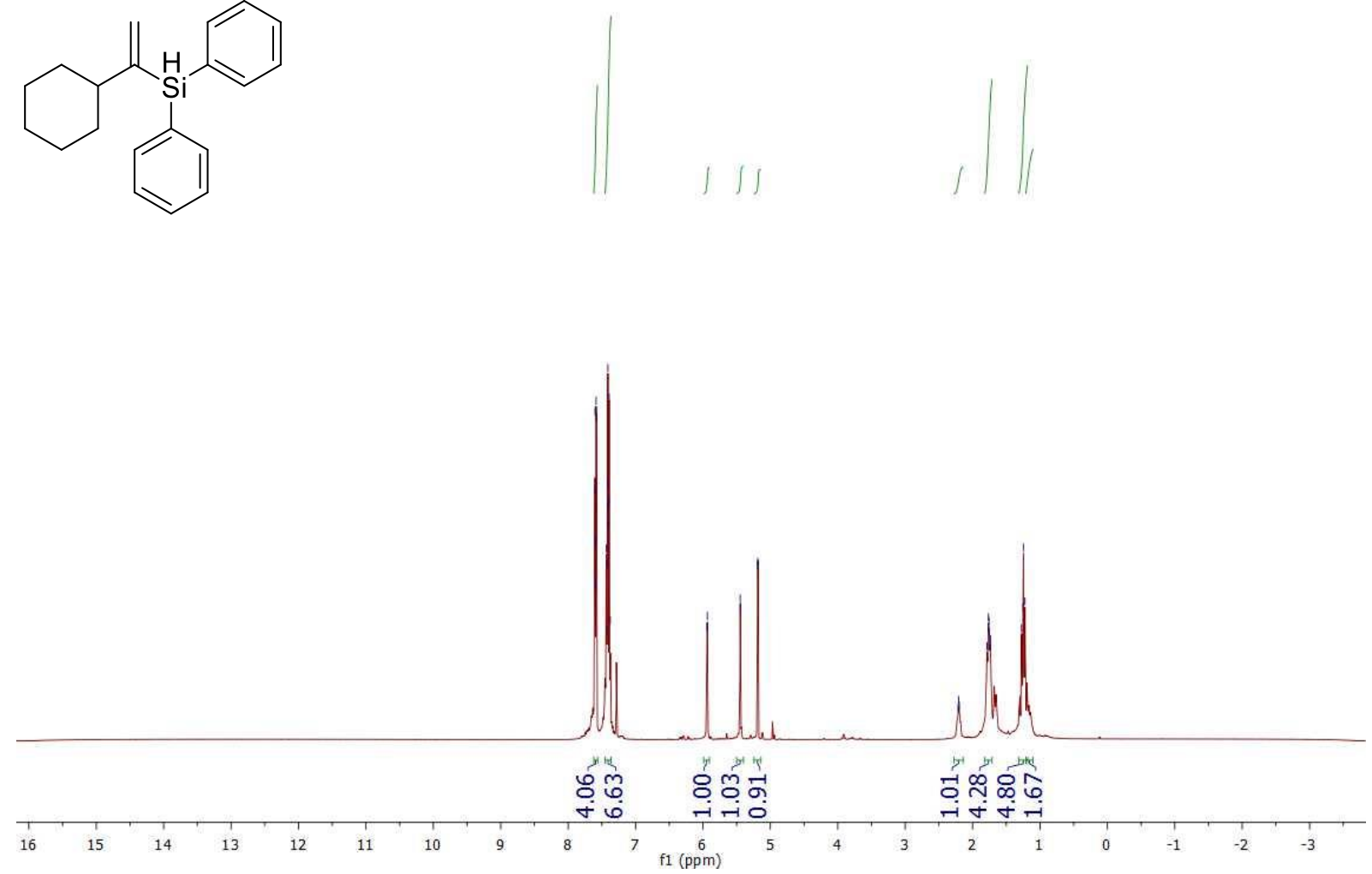

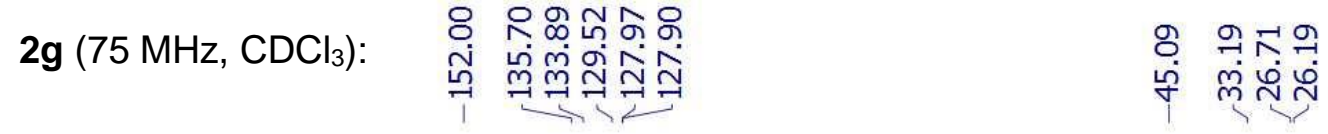<smiles>C=C(C1CCCCC1)[SiH](c1ccccc1)c1ccccc1</smiles>

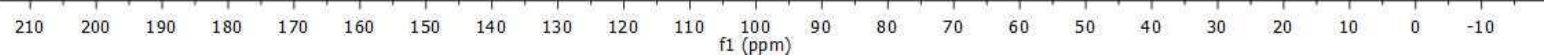


2h $\left(300 \mathrm{MHz}, \mathrm{CDCl}_{3}\right)$ :<smiles>C=C(C1CC1)[SiH](c1ccccc1)c1ccccc1</smiles>
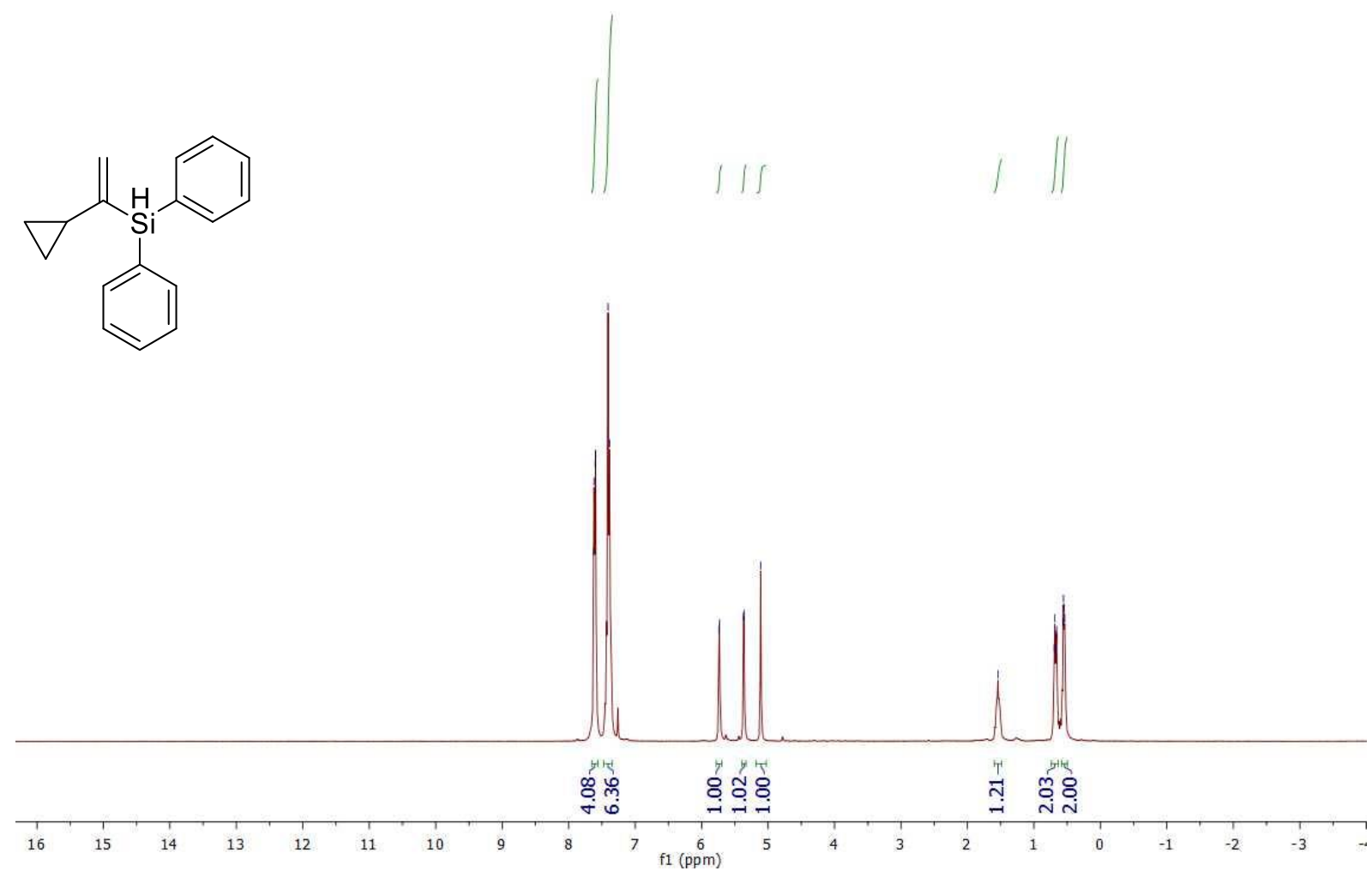

2h $\left(75 \mathrm{MHz}, \mathrm{CDCl}_{3}\right)$ :<smiles>C=C(C1CC1)[SiH](c1ccccc1)c1ccccc1</smiles>

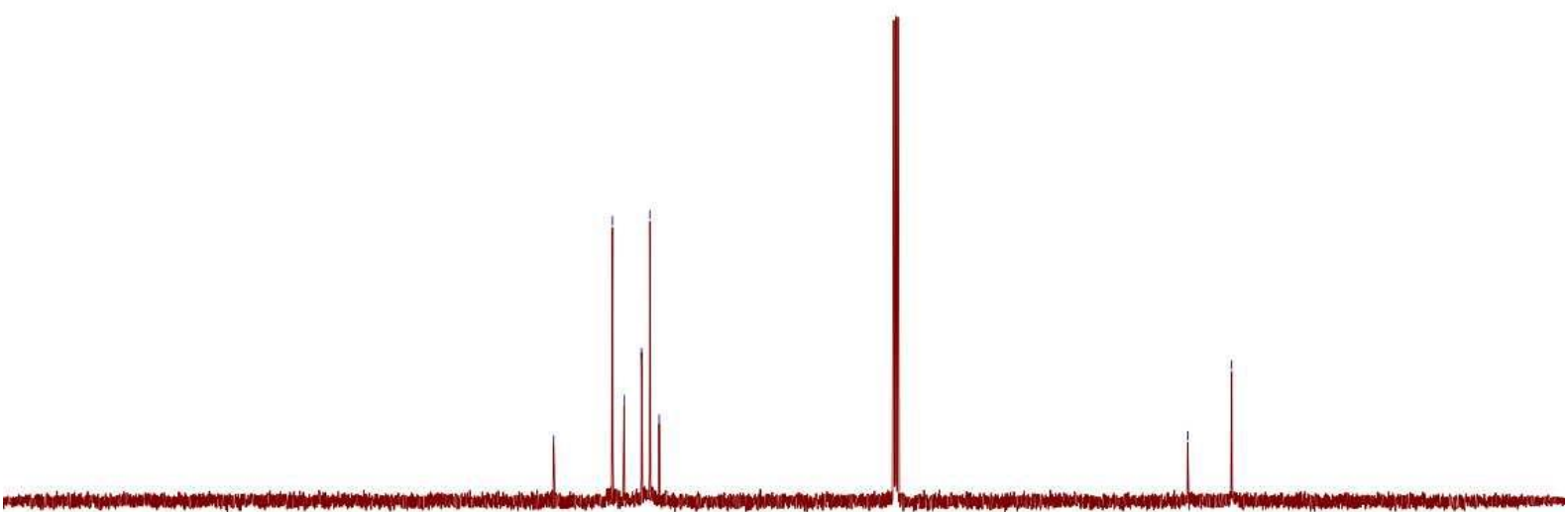

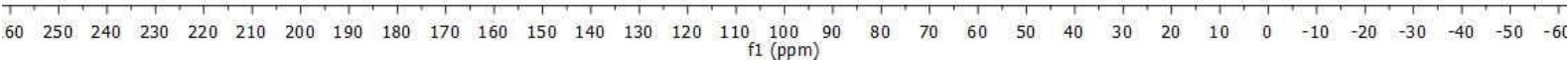


2i $\left(300 \mathrm{MHz}, \mathrm{CDCl}_{3}\right)$ :
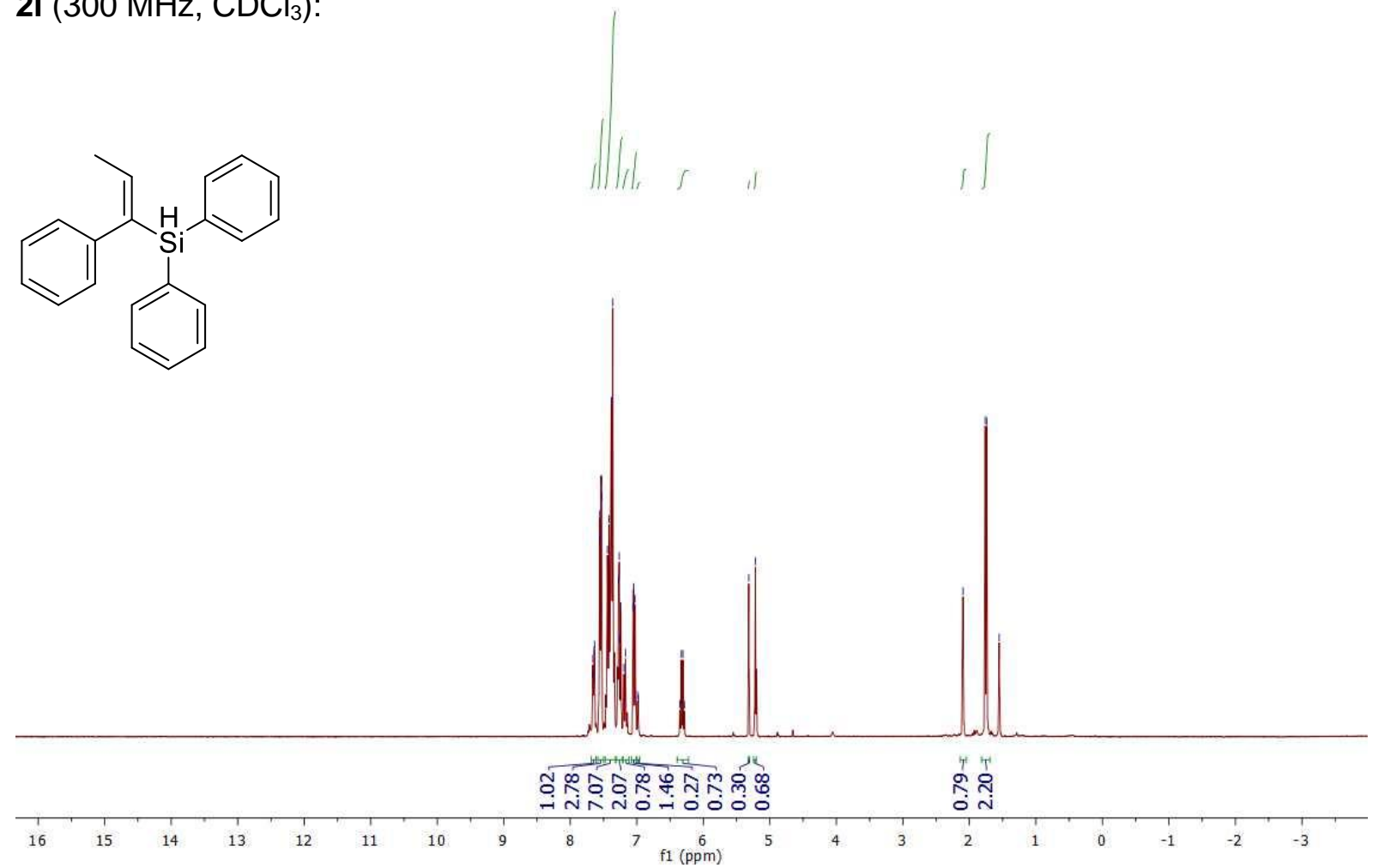

2i $\left(75 \mathrm{MHz}, \mathrm{CDCl}_{3}\right)$ :<smiles>C/C=C(\c1ccccc1)[SiH](c1ccccc1)c1ccccc1</smiles><smiles>CC(=Cc1ccccc1)[SiH](c1ccccc1)c1ccccc1</smiles>

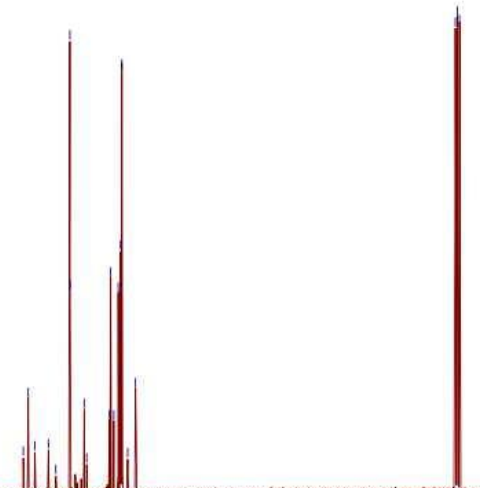




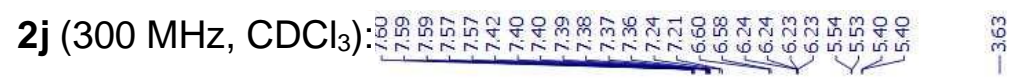<smiles>C=C(c1ccc(N)cc1)[SiH](c1ccccc1)c1ccccc1</smiles>
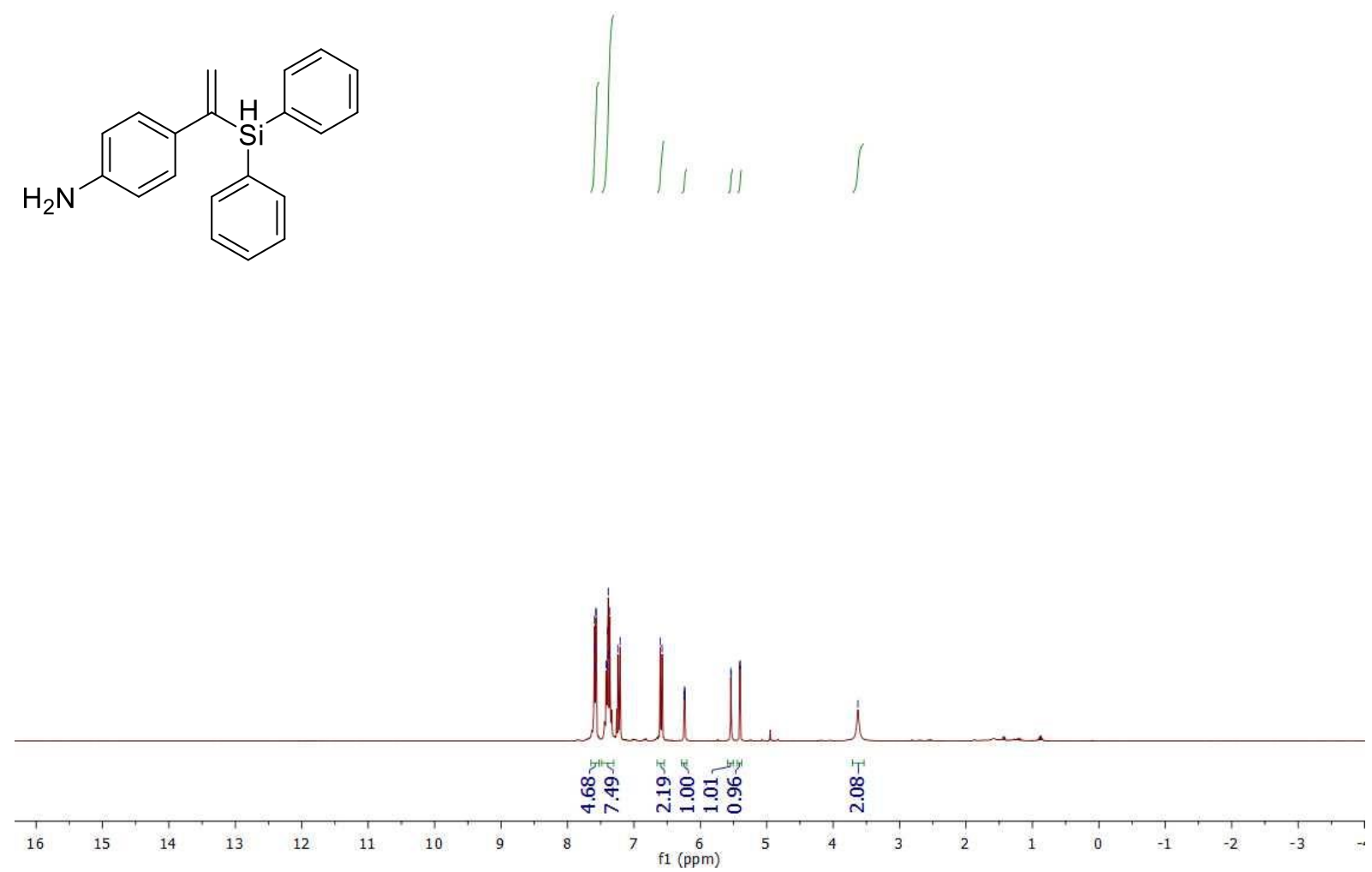

2j $\left(75 \mathrm{MHz}, \mathrm{CDCl}_{3}\right)$ :

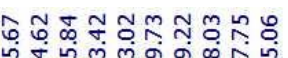

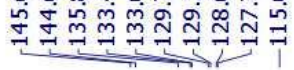<smiles>C=C(c1ccc(N)cc1)[SiH](c1ccccc1)c1ccccc1</smiles> 


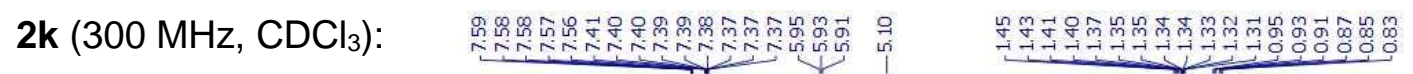<smiles>CCC/C=C(\CCC)[SiH](c1ccccc1)c1ccccc1</smiles>
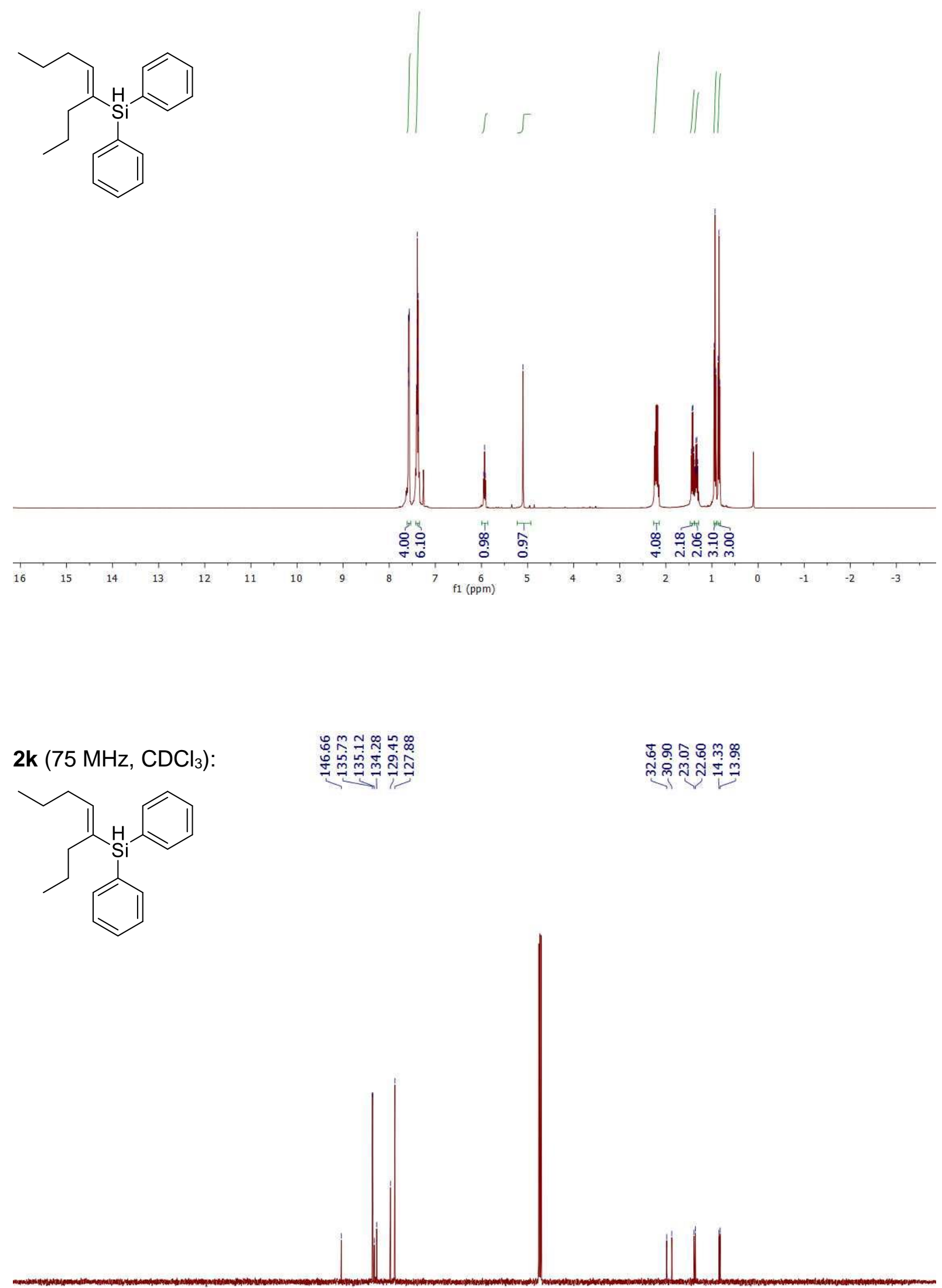

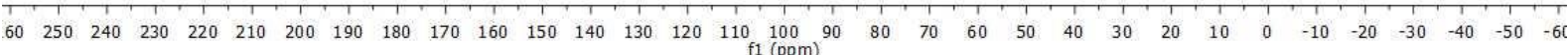




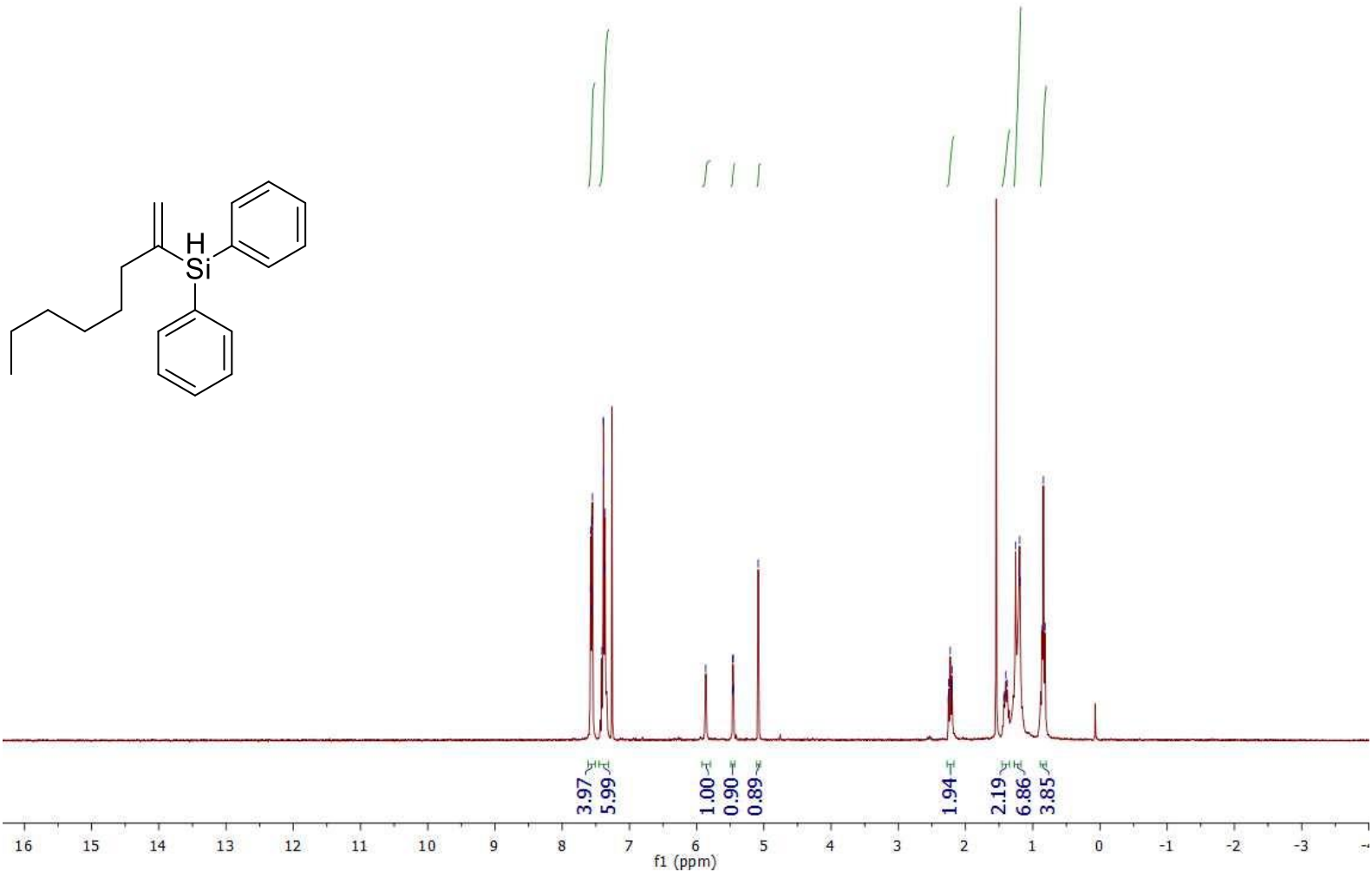

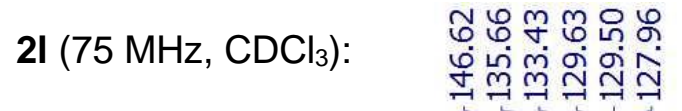<smiles>C=C(CCCCC)[SiH](c1ccccc1)c1ccccc1</smiles>

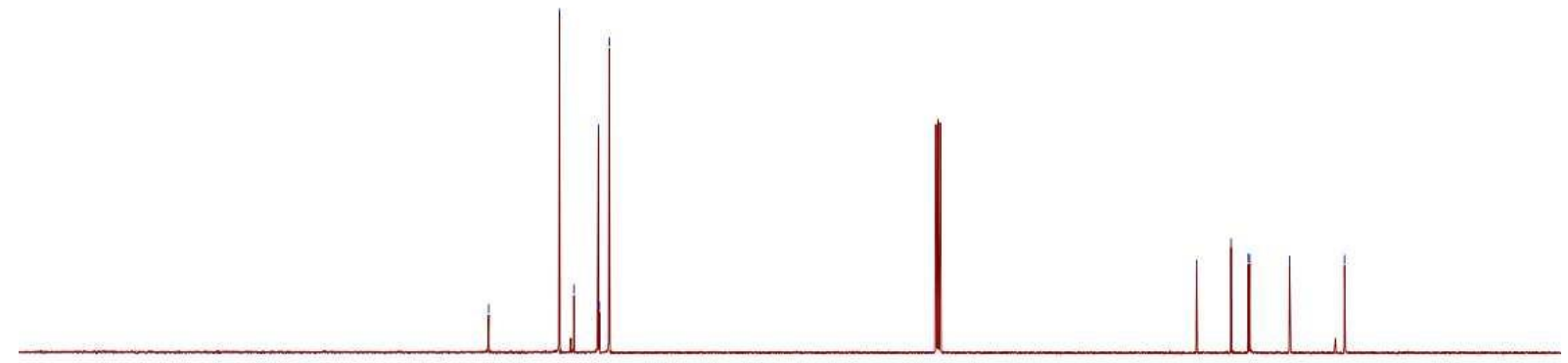

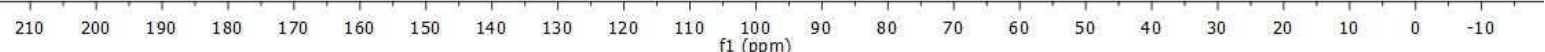


2 m $\left(300 \mathrm{MHz}, \mathrm{CDCl}_{3}\right)$ :

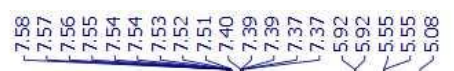

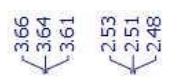

$\vec{m}$
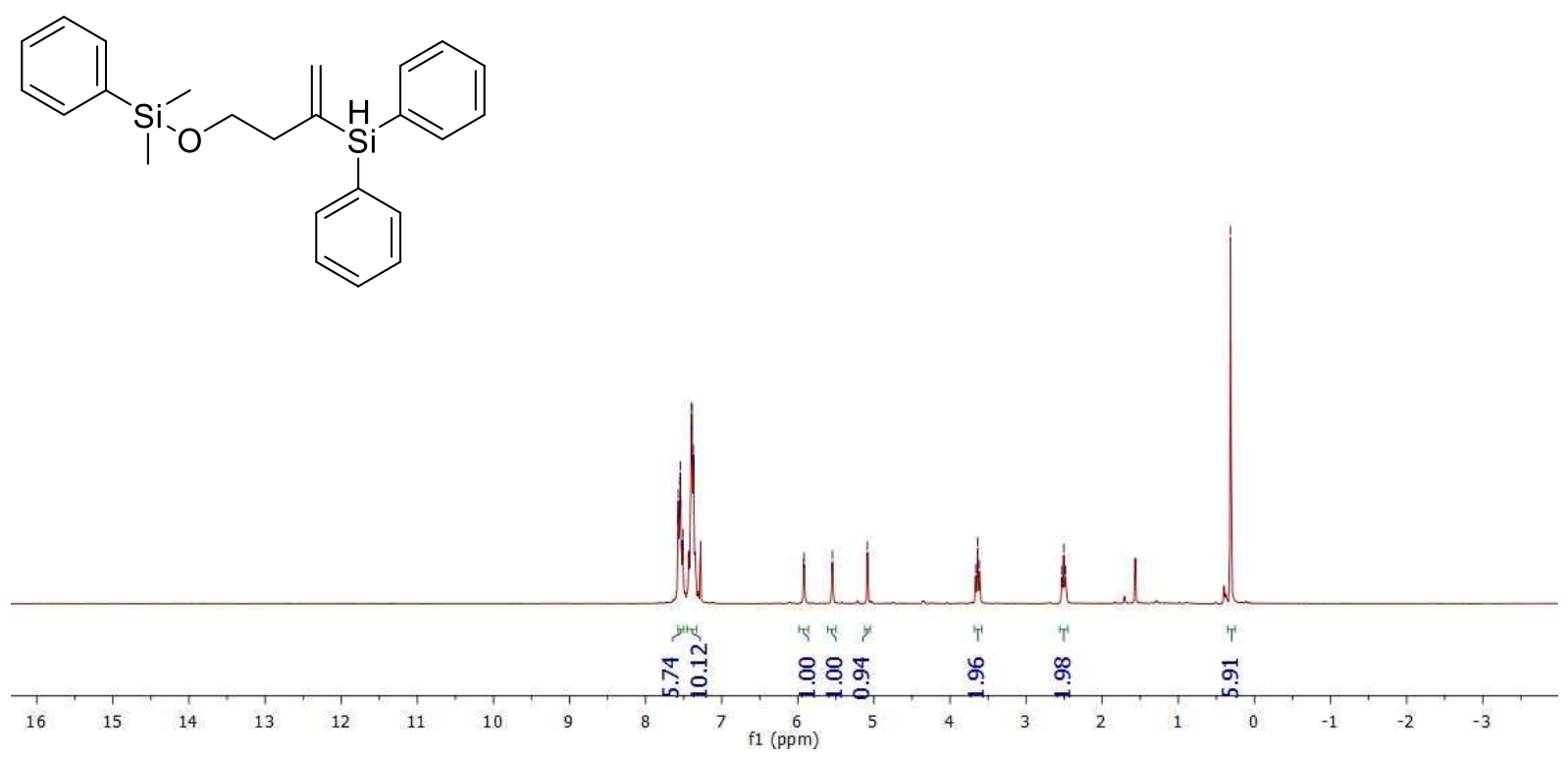

$2 \mathrm{~m}$ substrate $\left(300 \mathrm{MHz}, \mathrm{CDCl}_{3}\right)$ )

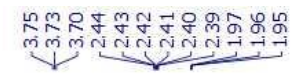
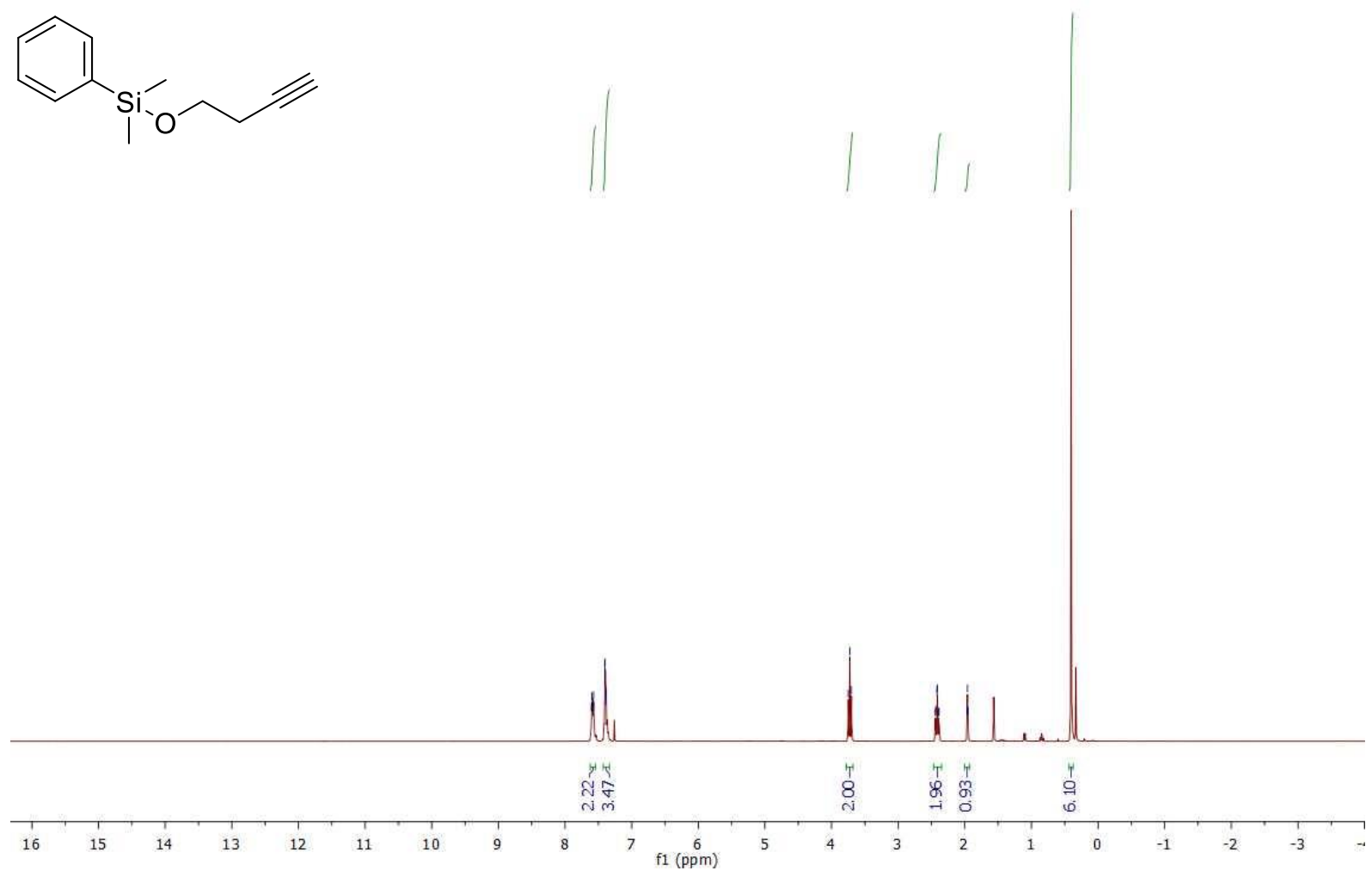

28 


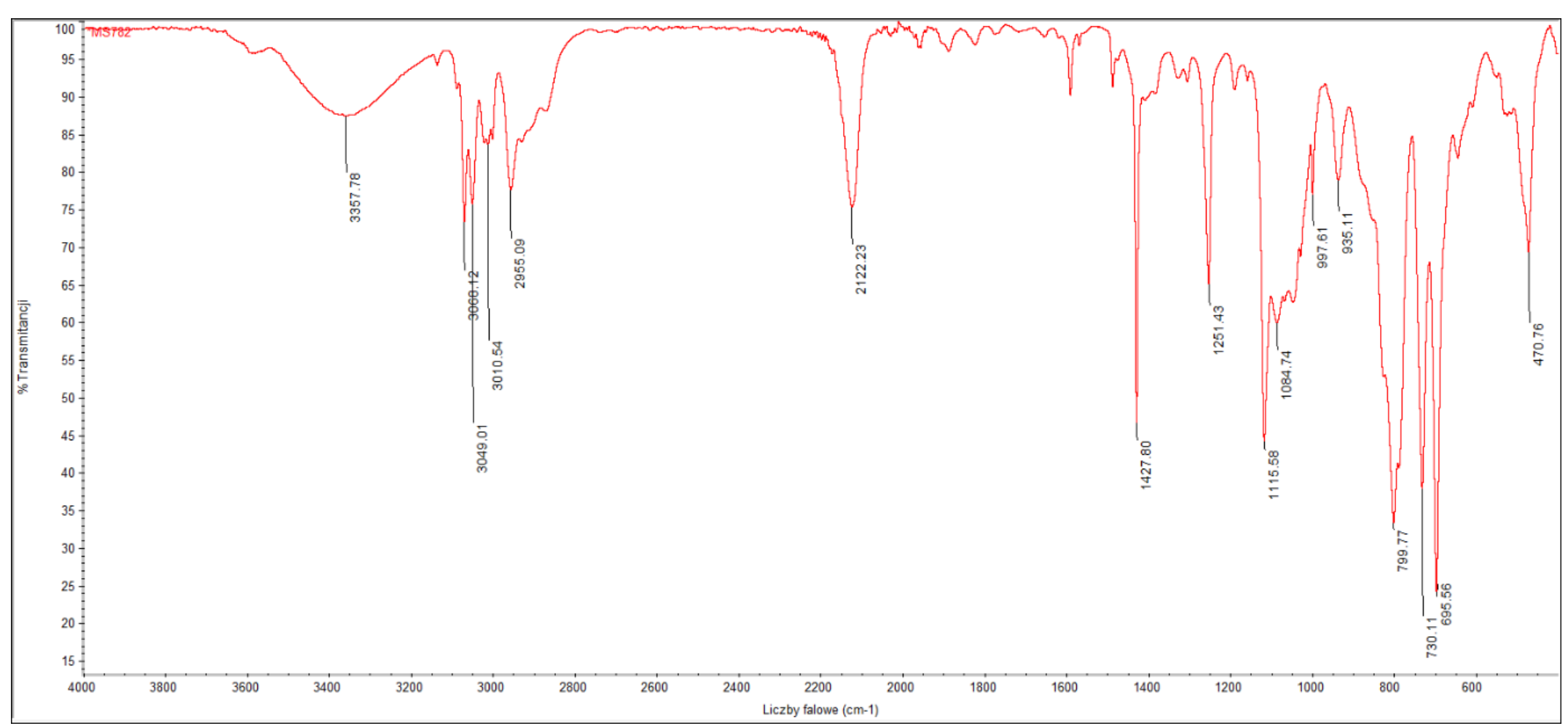




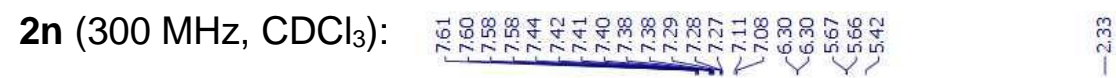<smiles>C=C(c1ccc(C)cc1)[SiH](c1ccccc1)c1ccccc1</smiles>
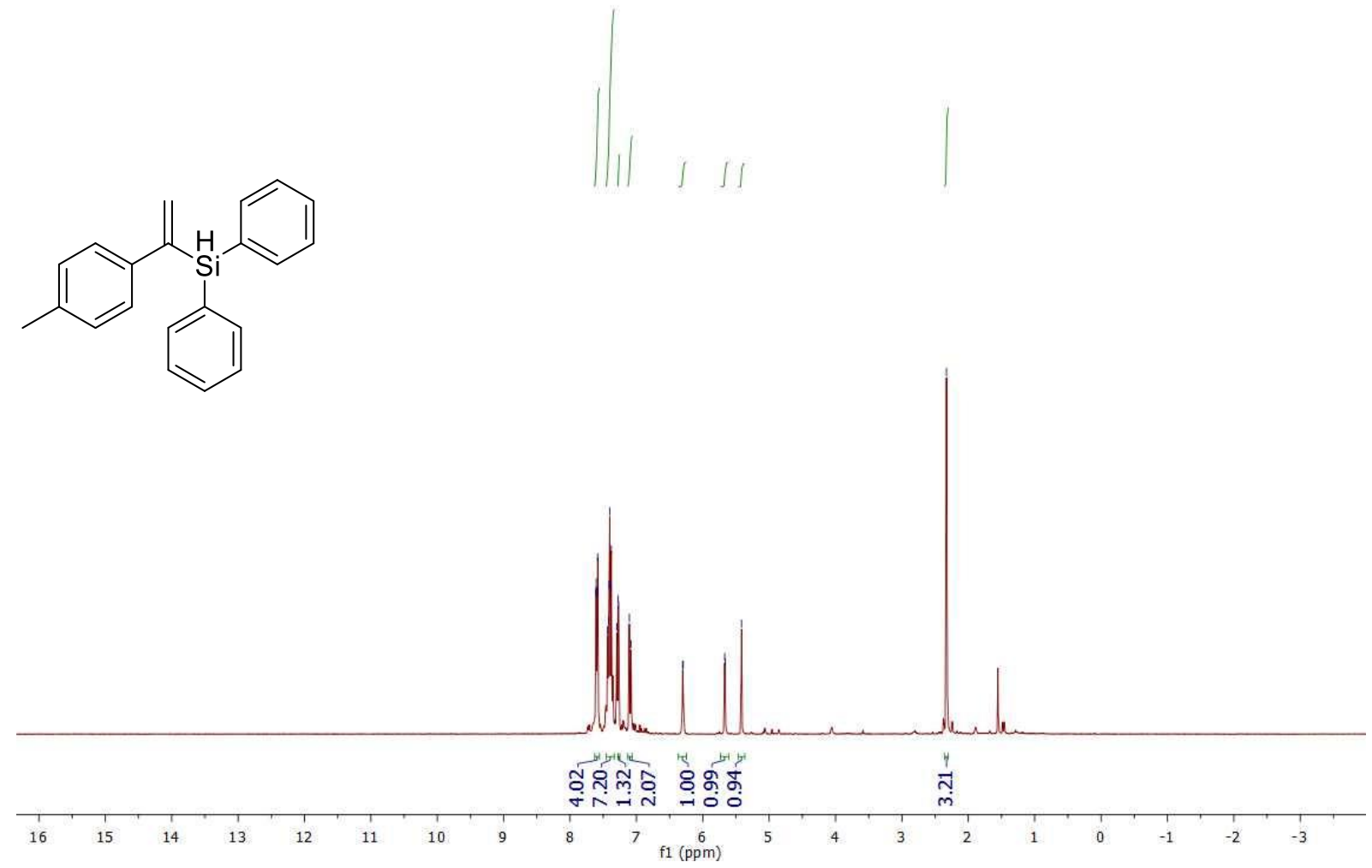

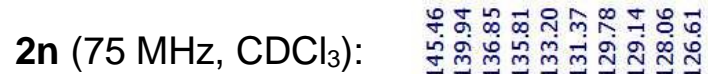

$\underset{i}{\stackrel{7}{i}}$<smiles>C=C(c1ccc(C)cc1)[SiH](c1ccccc1)c1ccccc1</smiles>

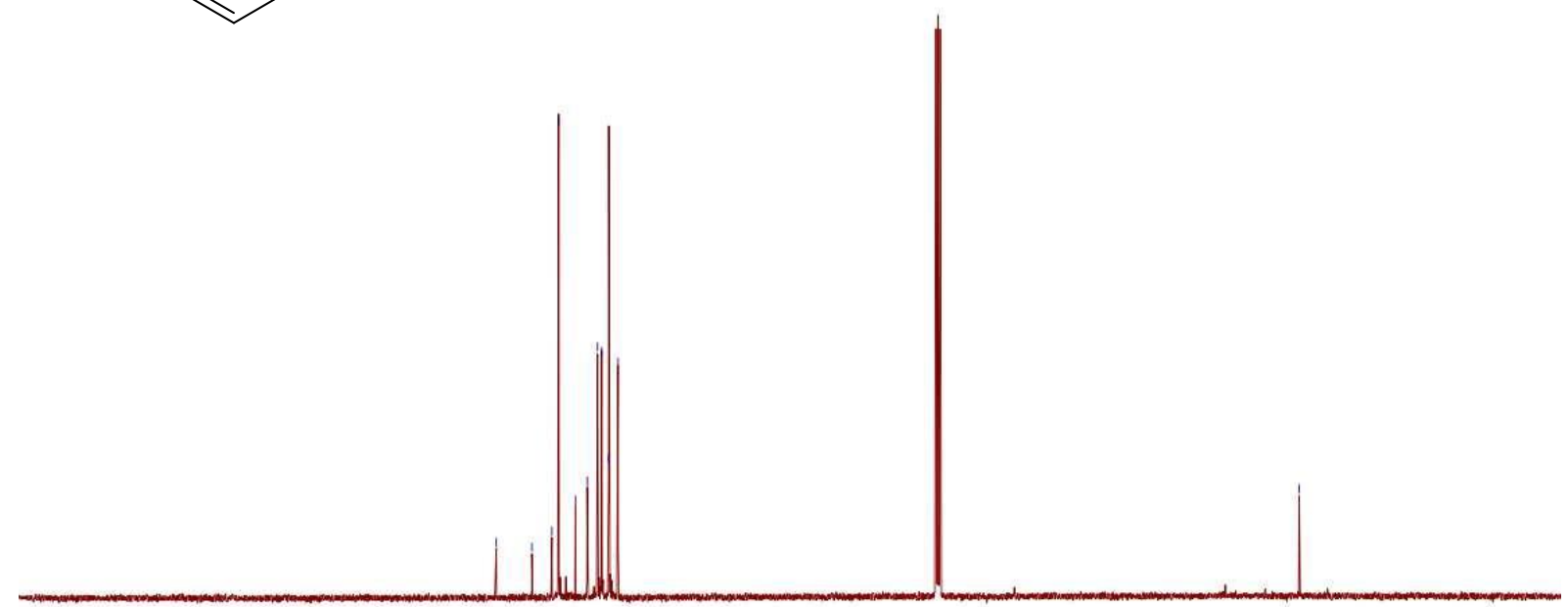

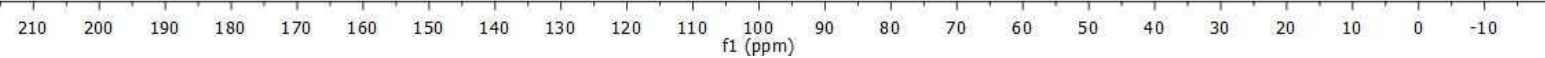




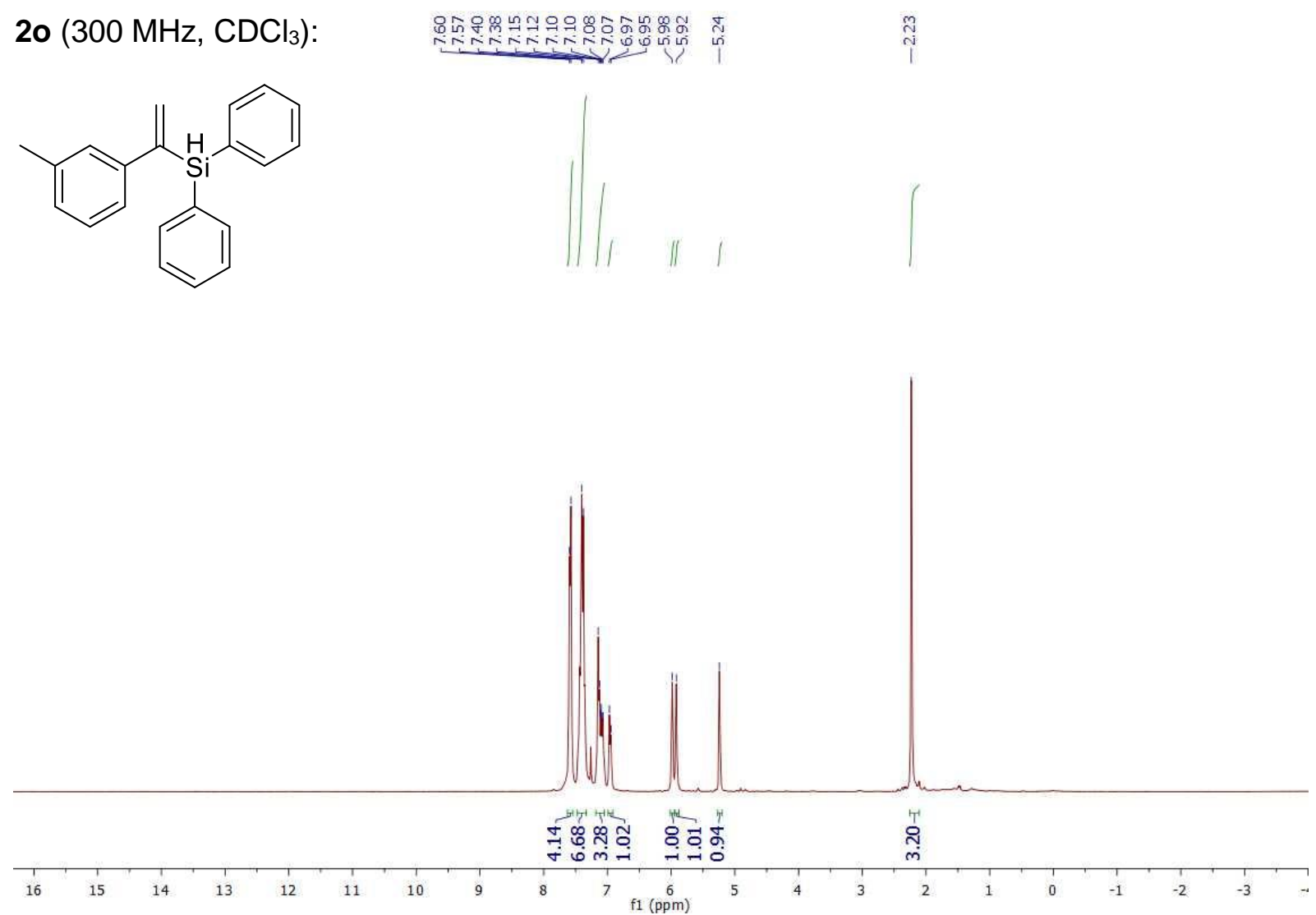

$20\left(75 \mathrm{MHz}, \mathrm{CDCl}_{3}\right)$ :<smiles>C=C(c1cccc(C)c1)[SiH](c1ccccc1)c1ccccc1</smiles>

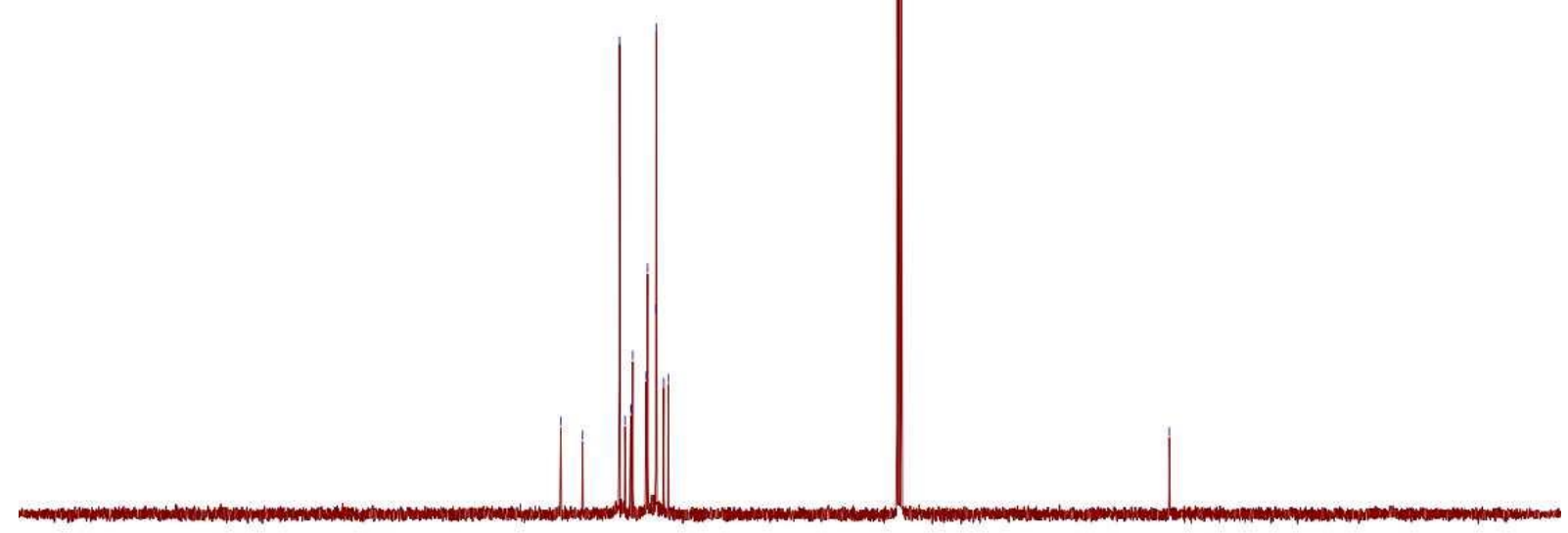

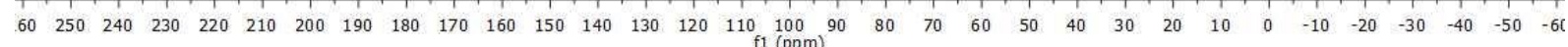


<smiles>C=C(c1ccccc1C)[SiH](c1ccccc1)c1ccccc1</smiles>
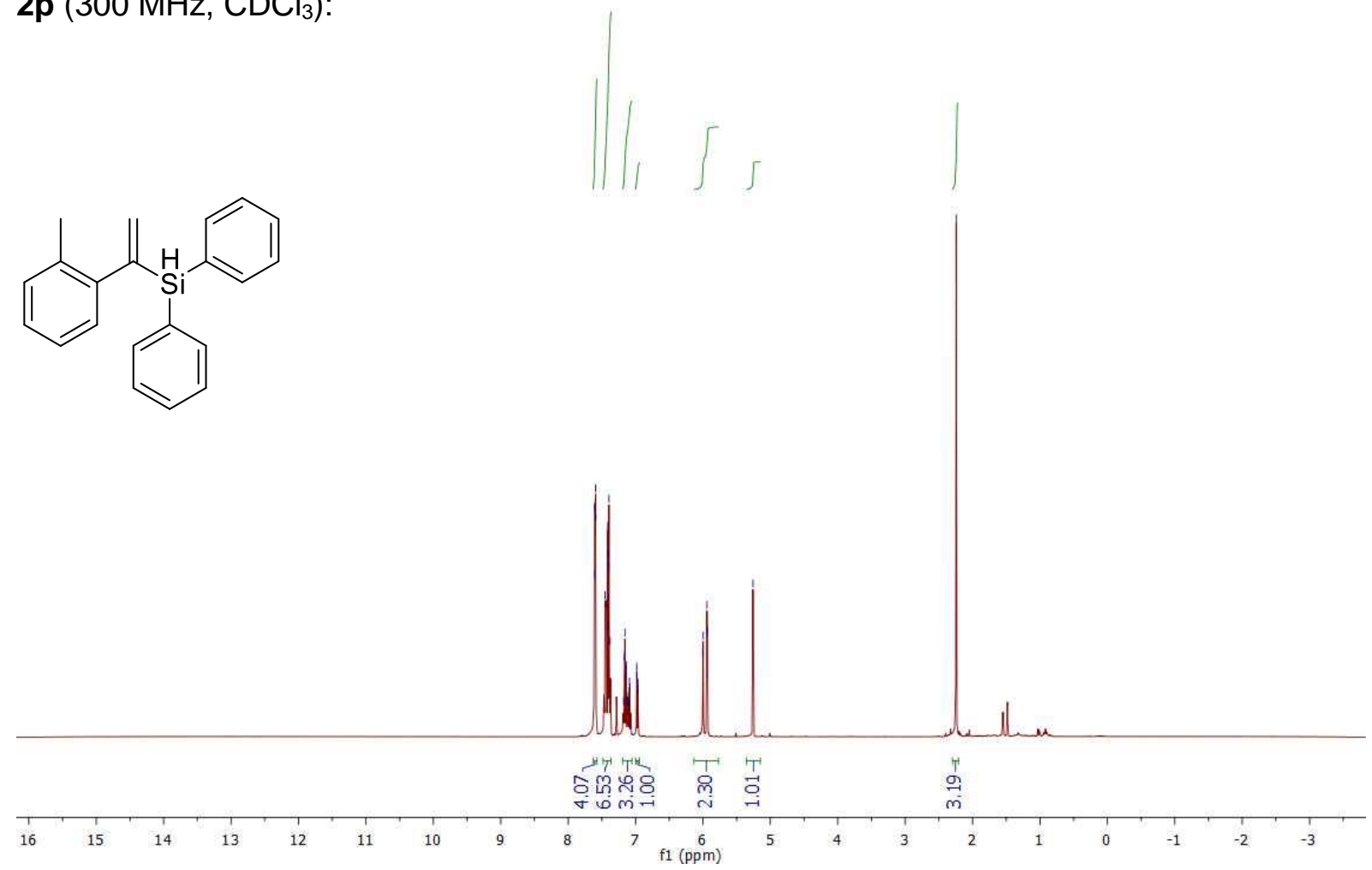

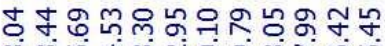

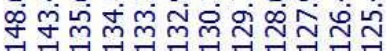

2p (75 MHz, $\left.\mathrm{CDCl}_{3}\right)$ :<smiles>C=C(c1ccccc1C)[SiH](c1ccccc1)c1ccccc1</smiles>

$210 \quad 200$

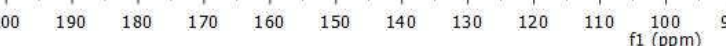

80

$70 \quad 60$ 


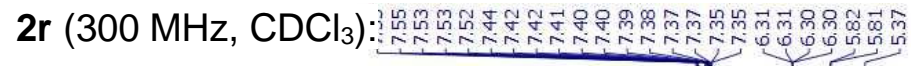<smiles>C=C(c1ccc(C#N)cc1)[SiH](c1ccccc1)c1ccccc1</smiles>
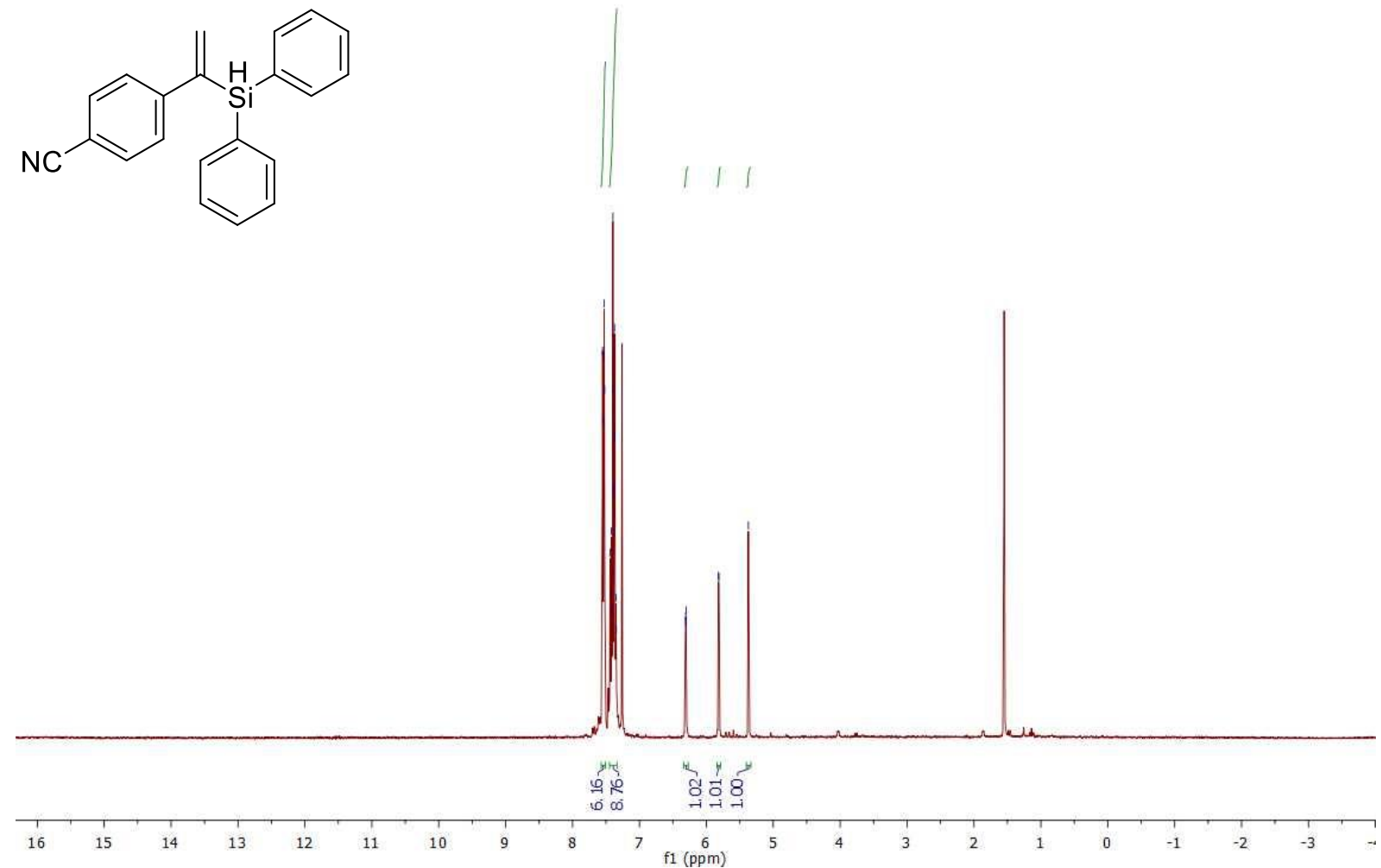

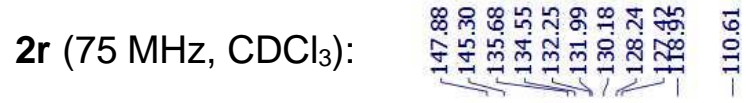<smiles>C=C(c1ccc(C#N)cc1)[SiH](c1ccccc1)c1ccccc1</smiles> 


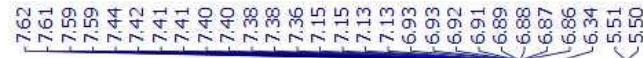

2s $\left(300 \mathrm{MHz}, \mathrm{CDCl}_{3}\right)$ :<smiles>C=C(c1ccsc1)[SiH](c1ccccc1)c1ccccc1</smiles>
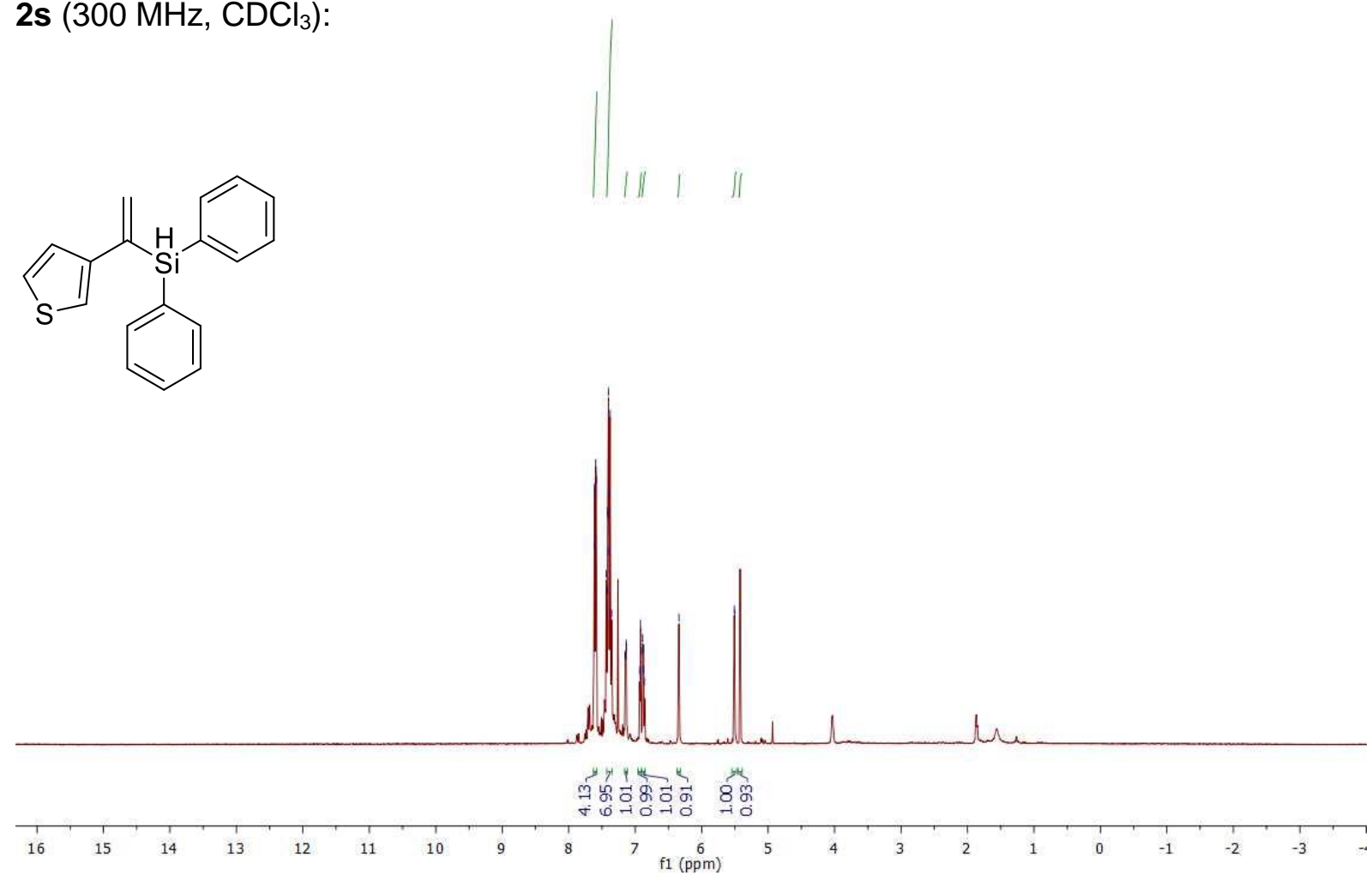

ติ

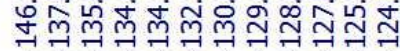

2s $\left(75 \mathrm{MHz} \mathrm{CDCl}_{3}\right)$ :<smiles>C=C(c1ccsc1)[SiH](c1ccccc1)c1ccccc1</smiles> 


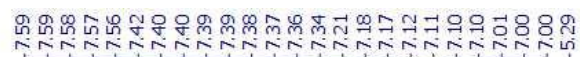

2t $\left(300 \mathrm{MHz}, \mathrm{CDCl}_{3}\right)$ :
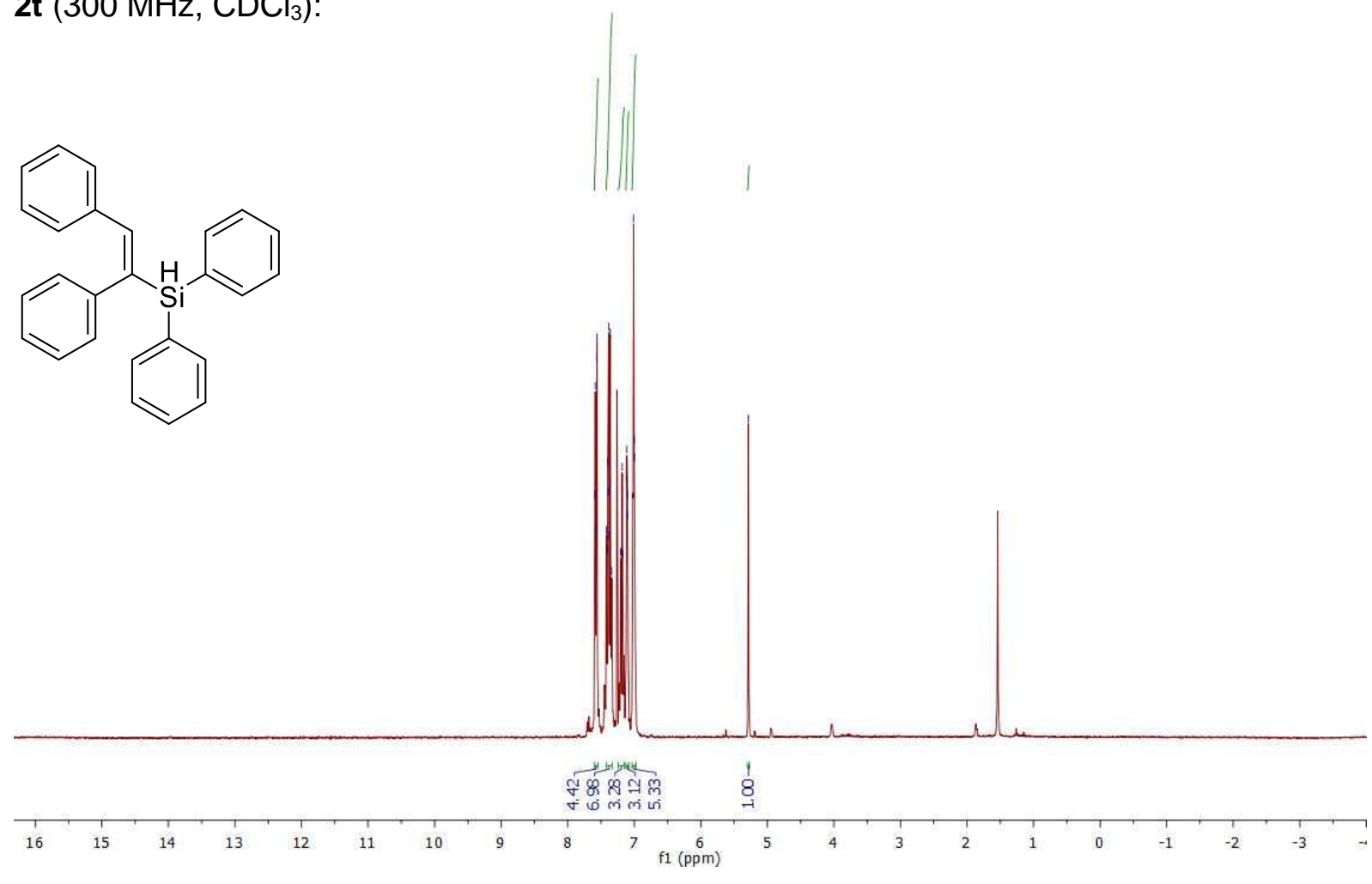

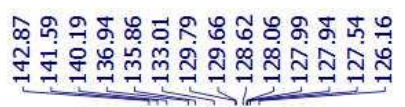

2t $\left(75 \mathrm{MHz}, \mathrm{CDCl}_{3}\right)$ :
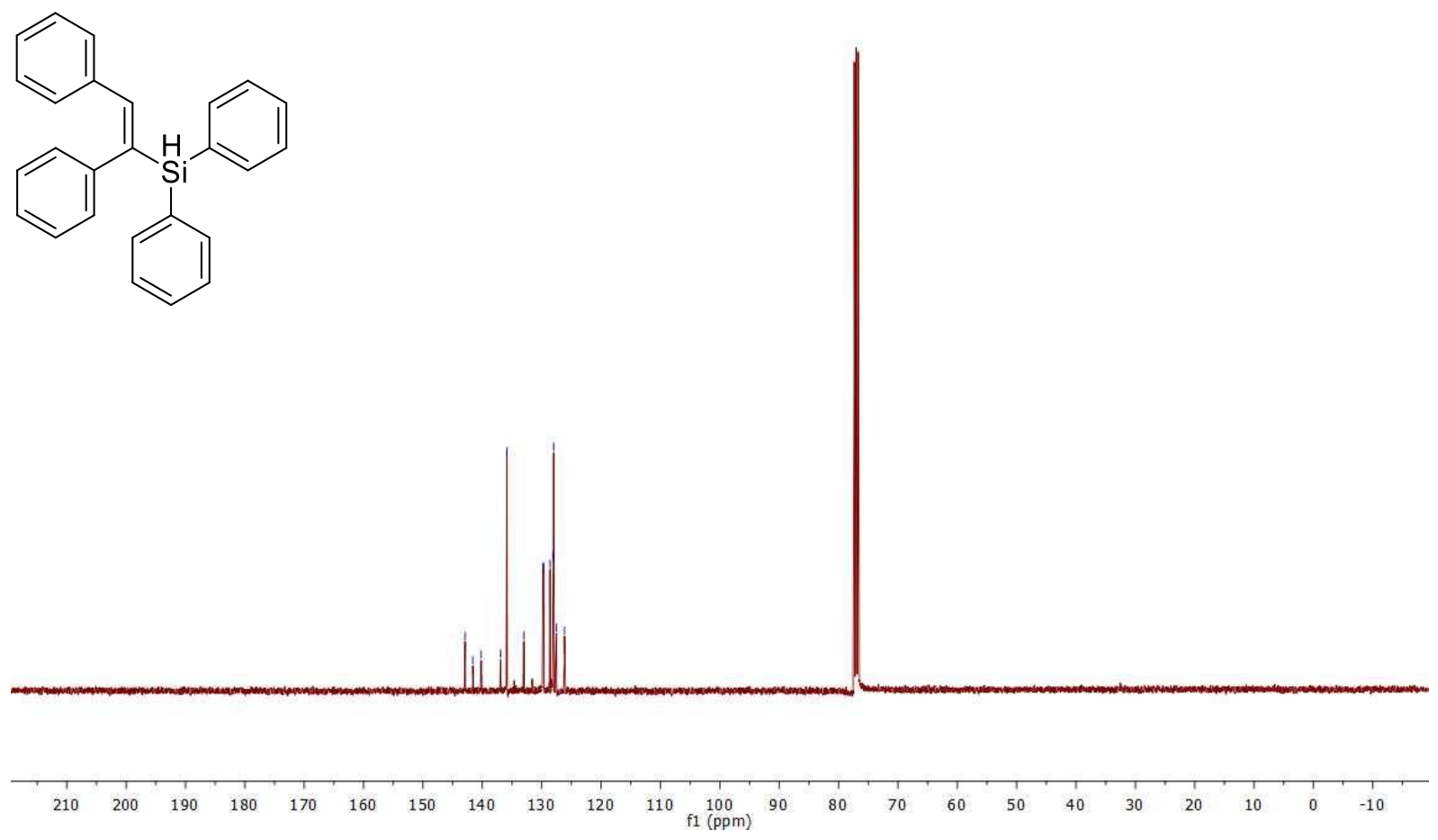

35 


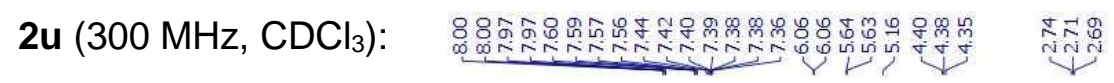<smiles>C=C(CCOC(=O)c1ccccc1)[SiH](c1ccccc1)c1ccccc1</smiles>
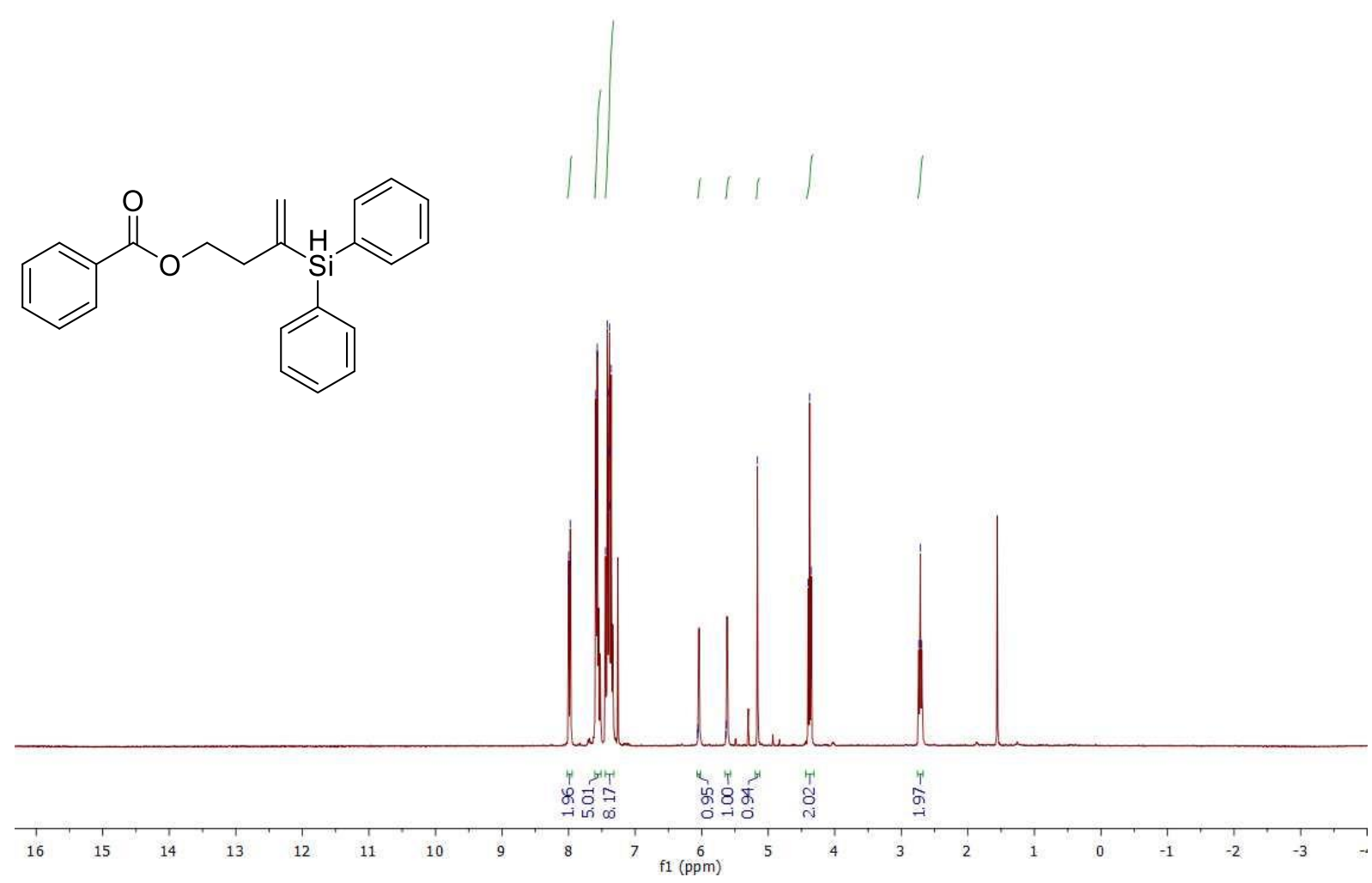

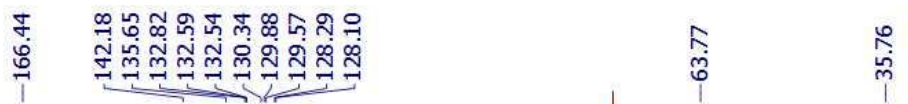

2u $\left(75 \mathrm{MHz}, \mathrm{CDCl}_{3}\right)$ :<smiles>C=C(CCOC(=O)c1ccccc1)[SiH](c1ccccc1)c1ccccc1</smiles>

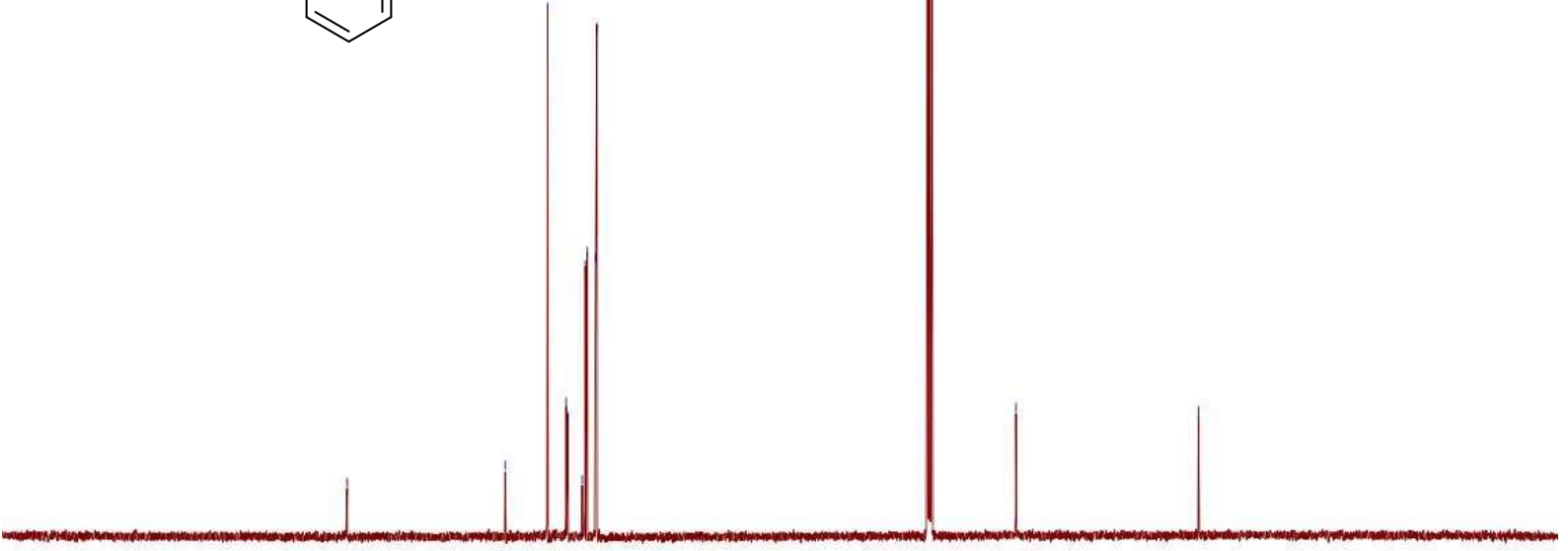

$\begin{array}{lllllllllllllllllllllllll}210 & 200 & 190 & 180 & 170 & 160 & 150 & 140 & 130 & 120 & 110 & 100 & 90 & 80 & 70 & 60 & 50 & 40 & 30 & 20 & 10 & 0 & -10 & \end{array}$ 


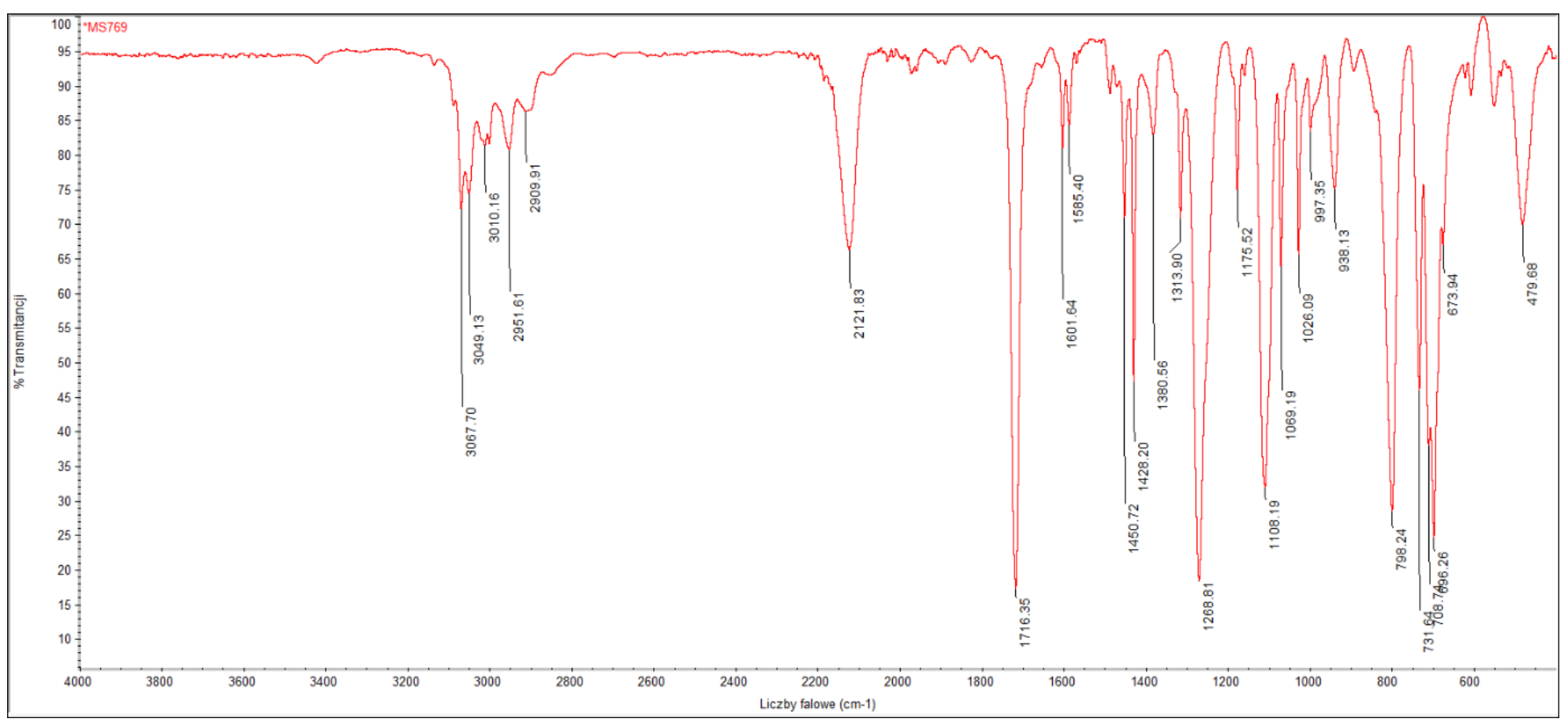




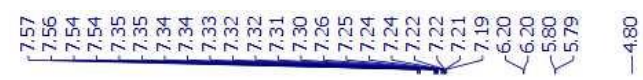

3a $\left(300 \mathrm{MHz}, \mathrm{CDCl}_{3}\right)$ :

$\approx$
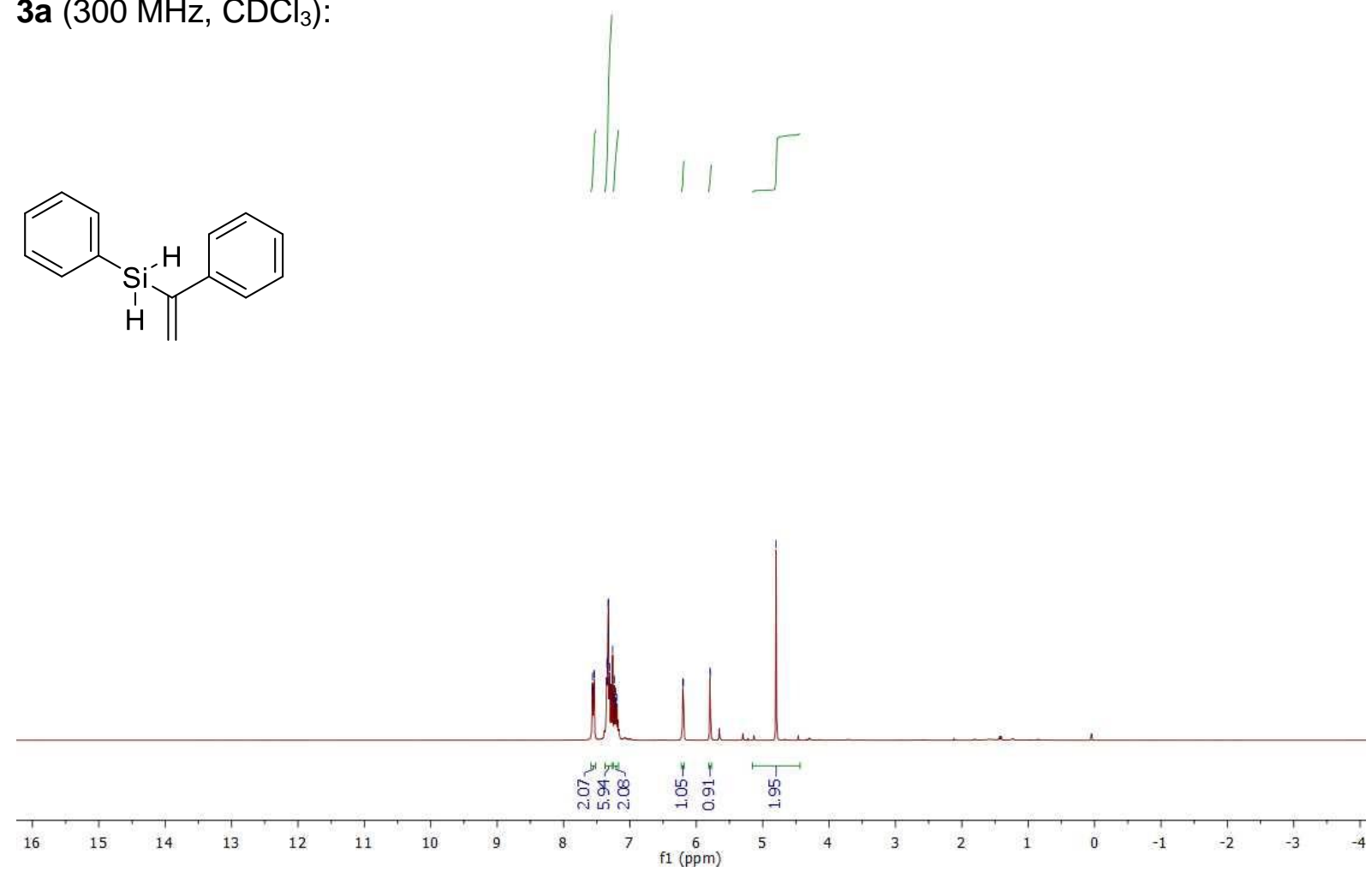

3a $\left(75 \mathrm{MHz}, \mathrm{CDCl}_{3}\right): \quad$ :<smiles>C=C([SiH]c1ccccc1)c1ccccc1</smiles>

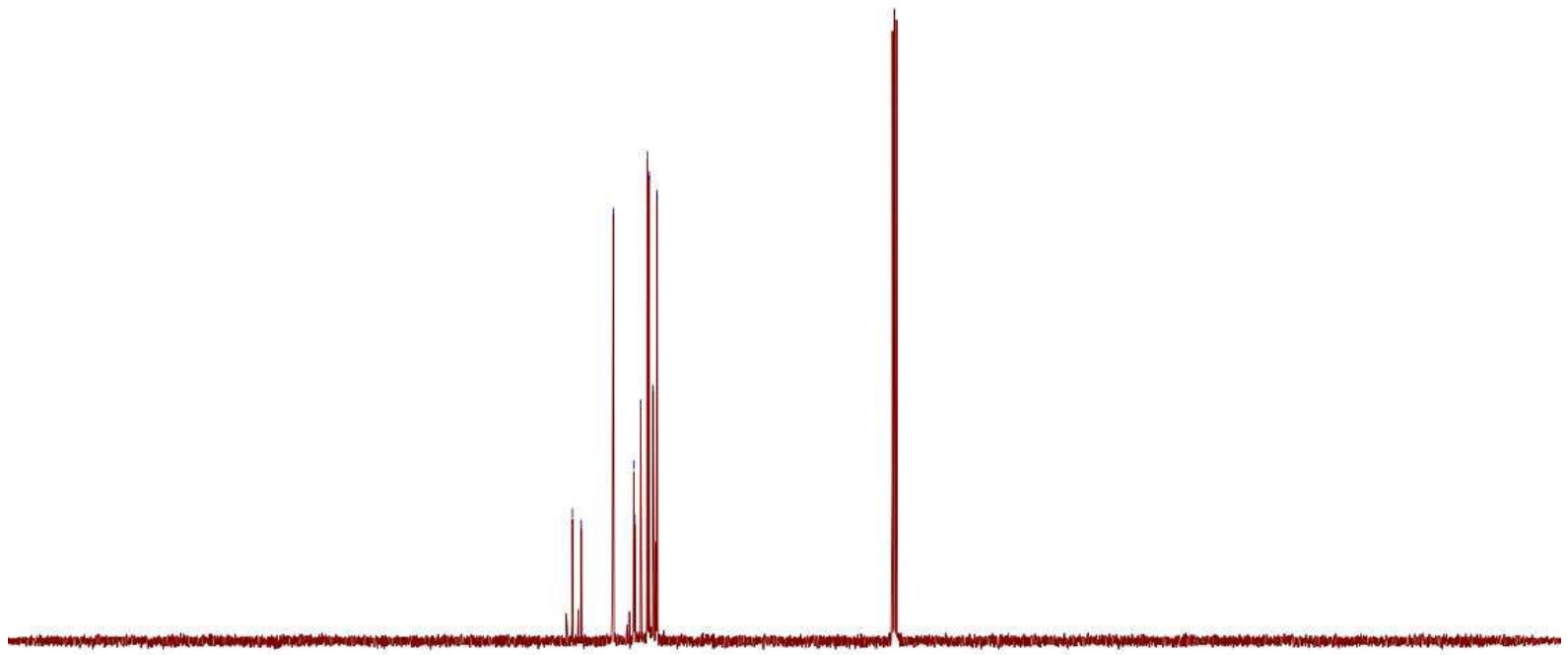

$\begin{array}{llllllllllllllllllllllllllllllllll}60 & 250 & 240 & 230 & 220 & 210 & 200 & 190 & 180 & 170 & 160 & 150 & 140 & 130 & 120 & 110 & 100 & 90 & 8 & 70 & 60 & 50 & 40 & 30 & 20 & 10 & 0 & -10 & -20 & -30 & -40 & -50 & -66\end{array}$ 


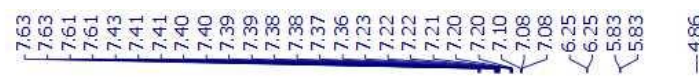

$\underset{\substack{0 \\ \text { i }}}{1}$

3g (300 MHz, $\left.\mathrm{CDCl}_{3}\right)$ :
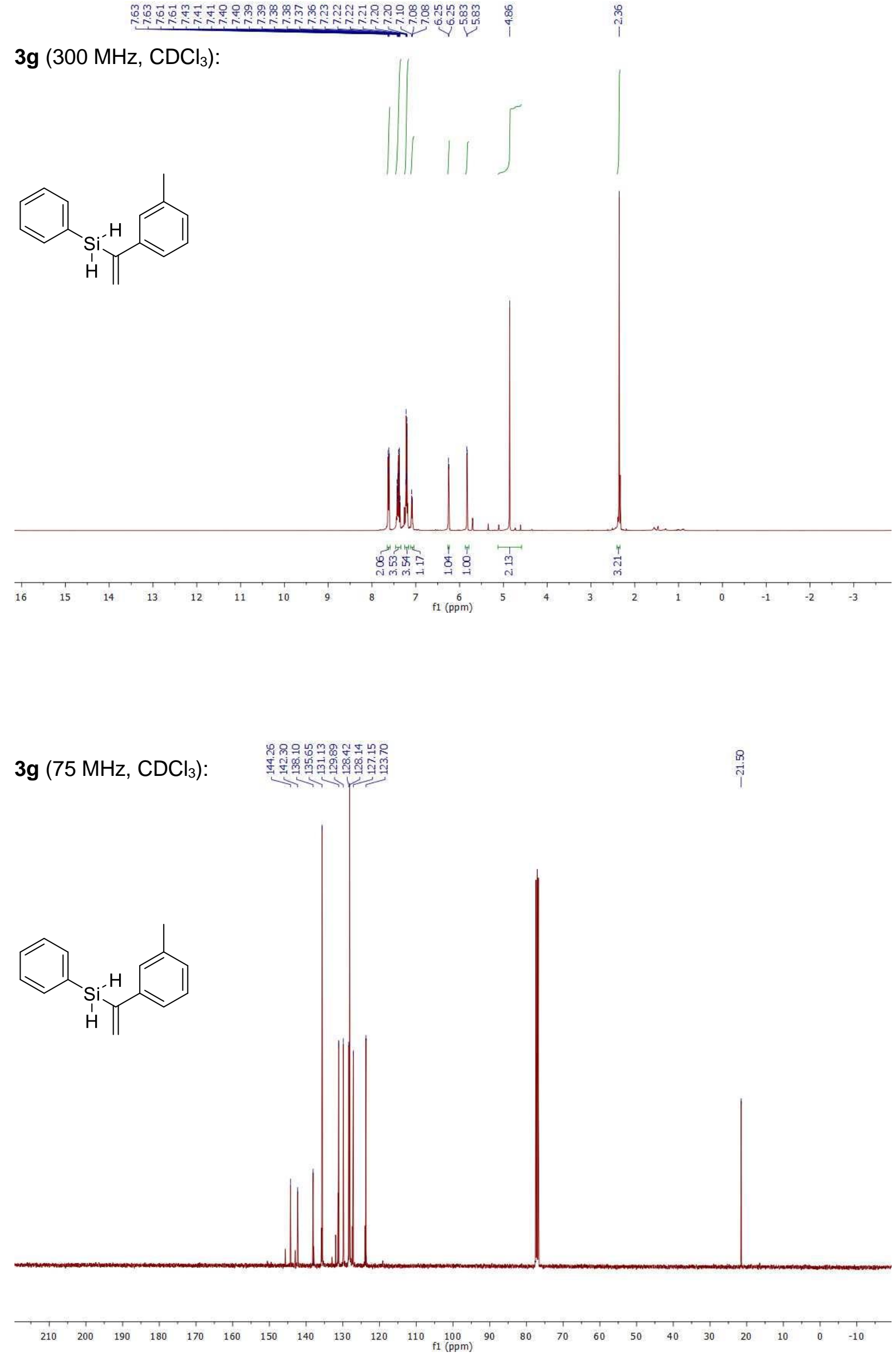

39 


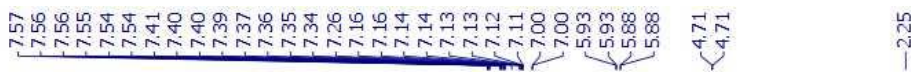

3h (300 MHz, $\left.\mathrm{CDCl}_{3}\right)$ :<smiles>C=C([SiH2]c1ccccc1)c1ccccc1C</smiles>
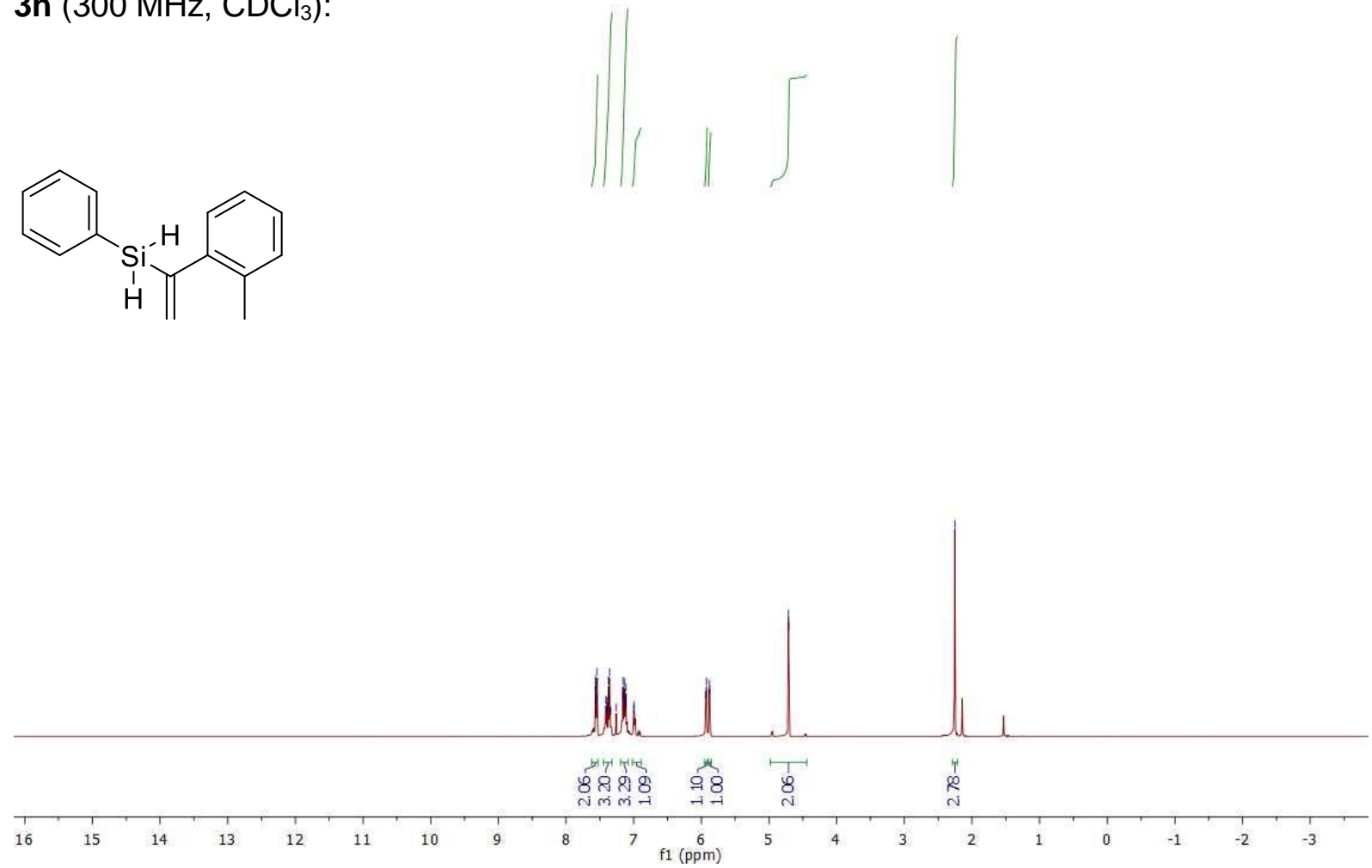

3h $\left(75 \mathrm{MHz}, \mathrm{CDCl}_{3}\right): \quad$ :<smiles>C=C([SiH]c1ccccc1)c1ccccc1C</smiles>

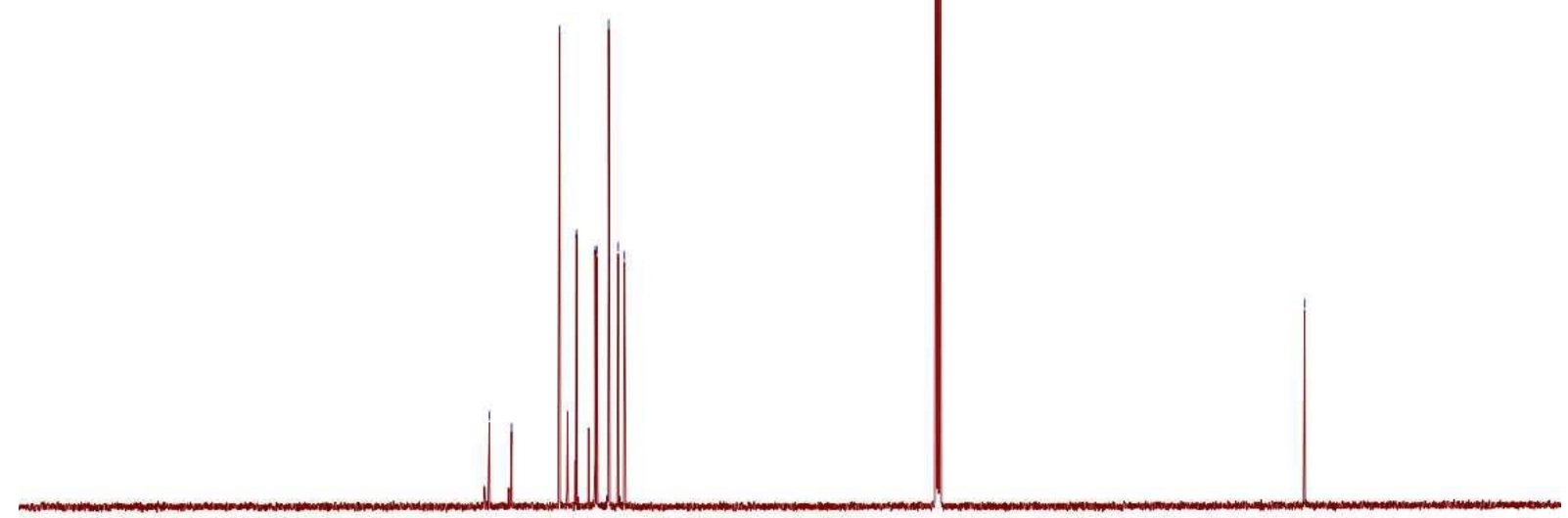

$\begin{array}{lllllllllllllllllllllllllllllllll}210 & 200 & 190 & 180 & 170 & 160 & 150 & 140 & 130 & 120 & 110 & 100 & 90 & 80 & 70 & 60 & 50 & 40 & 30 & 20 & 10 & 0 & -10\end{array}$ 
4a (300 MHz, $\left.\mathrm{CDCl}_{3}\right)$ :<smiles>C=C(c1ccccc1)[Si](C)(C)c1ccccc1</smiles>
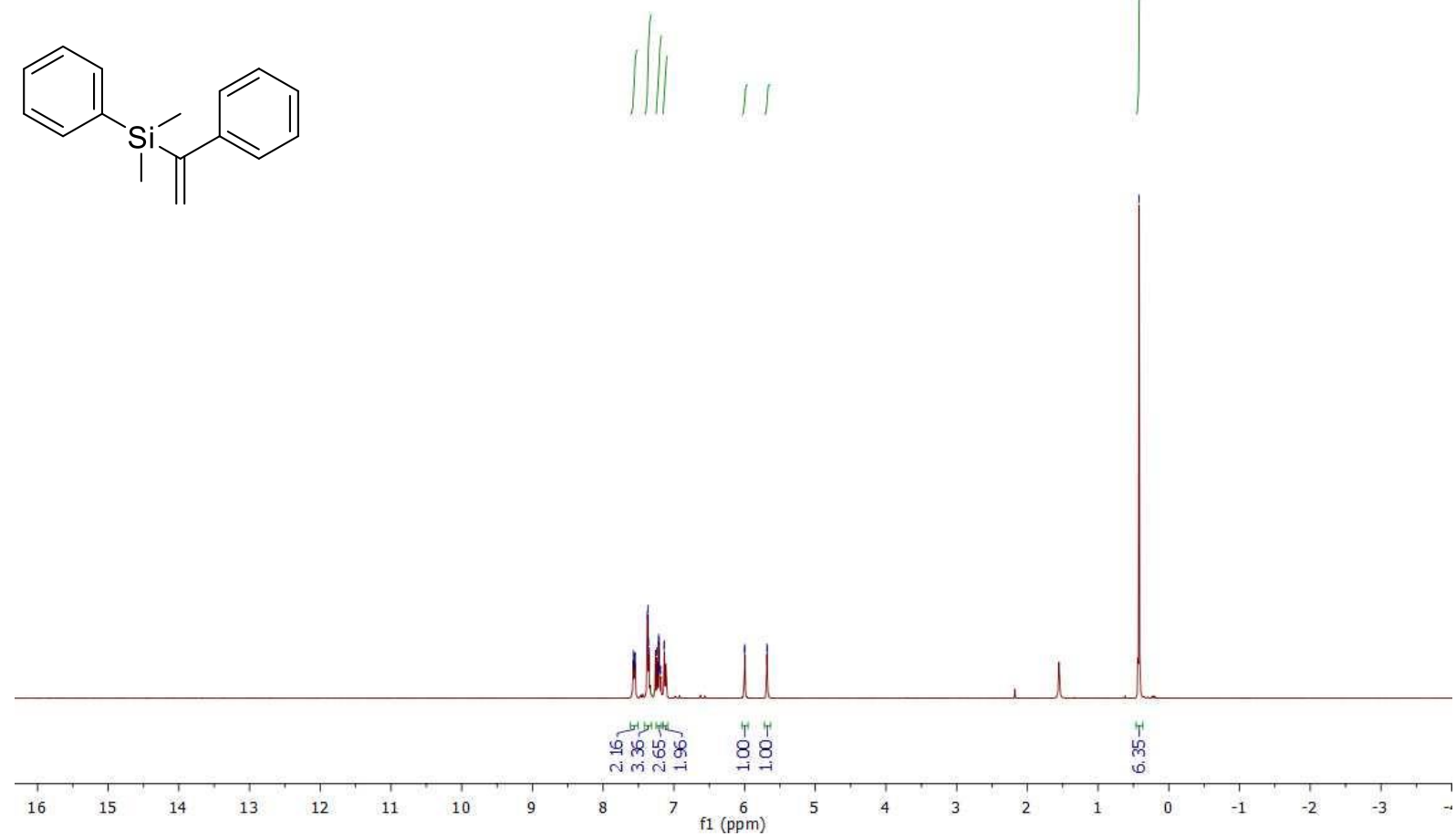<smiles>C=C(c1ccccc1)[Si](C)(C)c1ccccc1</smiles>

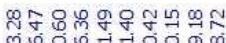

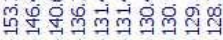

${ }^{2}$ 


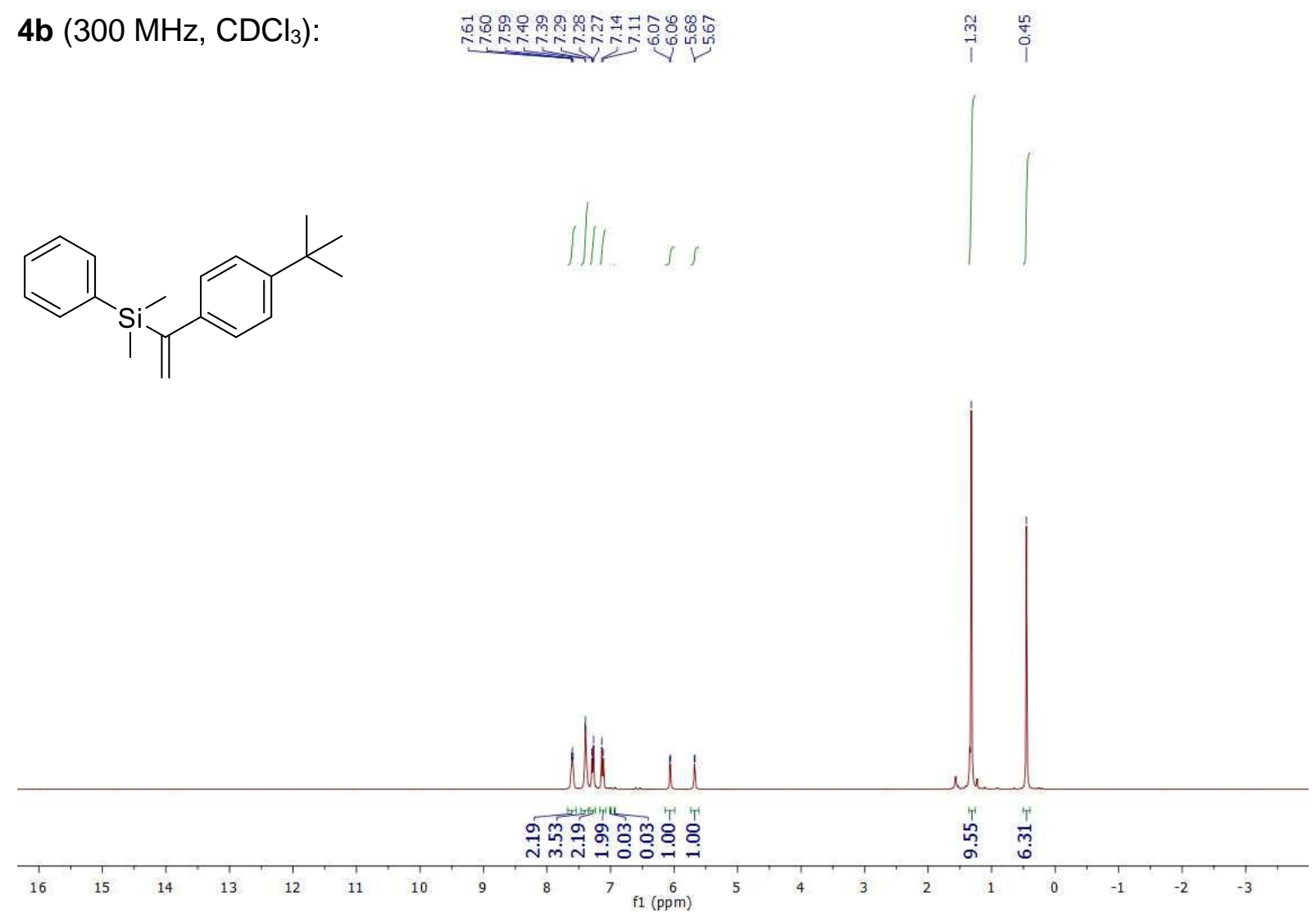

\begin{tabular}{|c|}
\hline 4b $\left(75 \mathrm{MHz} \mathrm{CDCl}_{3}\right):$ \\
\hline
\end{tabular}<smiles>C=C(c1ccc(C(C)(C)C)cc1)[Si](C)(C)c1ccccc1</smiles>

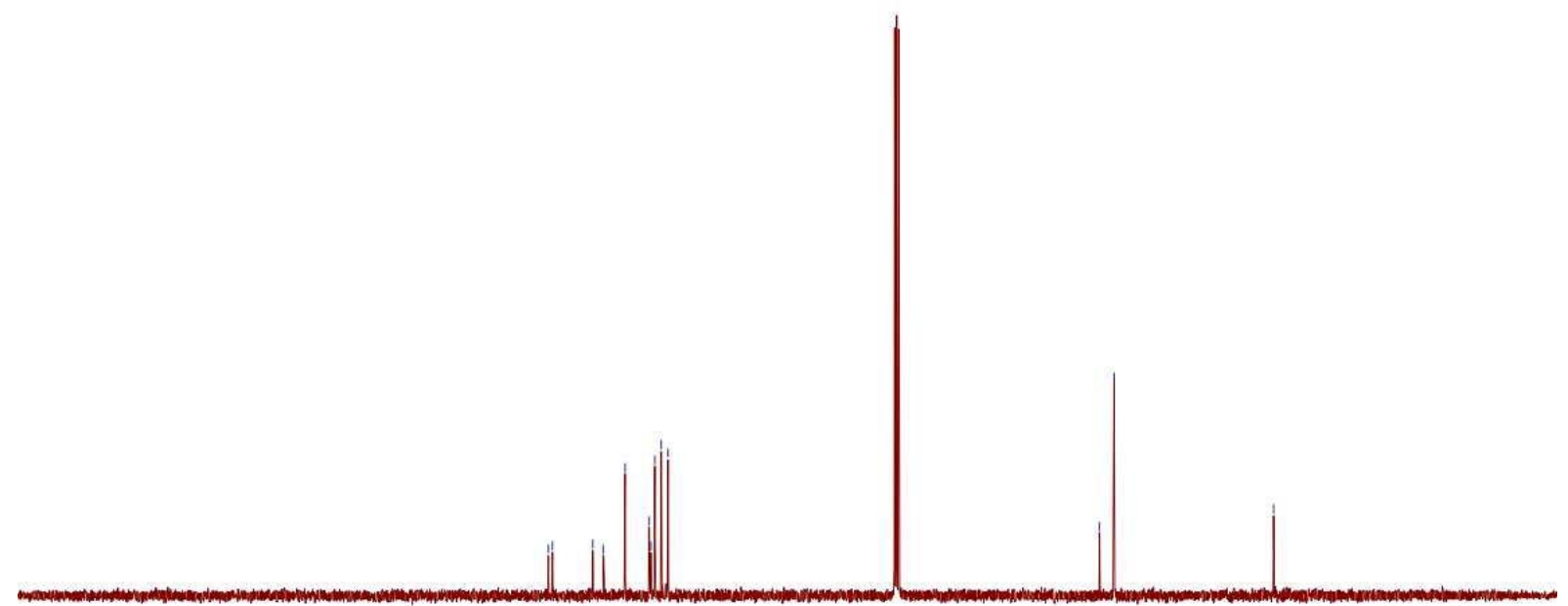

\begin{tabular}{rllllllllllllllllllllllllllllllll}
\hline 260 & 250 & 240 & 230 & 220 & 210 & 200 & 190 & 180 & 170 & 160 & 150 & 140 & 130 & 120 & 110 & 100 & 90 & 80 & 70 & 60 & 50 & 40 & 30 & 20 & 10 & 0 & -10 & -20 & -30 & -40 & -50 &
\end{tabular} 
4c $\left(300 \mathrm{MHz}, \mathrm{CDCl}_{3}\right)$ :

$\overbrace{i=1}^{\mathrm{Br}}$

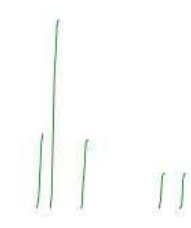

$$
1
$$

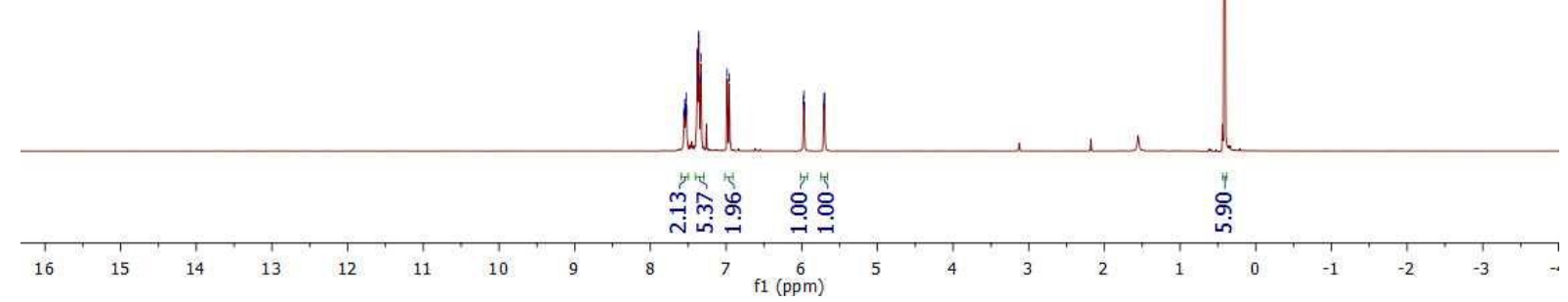

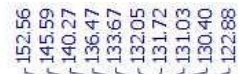

4c $\left(75 \mathrm{MHz}, \mathrm{CDCl}_{3}\right)$ :<smiles>C=C(c1ccc(Br)cc1)[Si](C)(C)c1ccccc1</smiles>

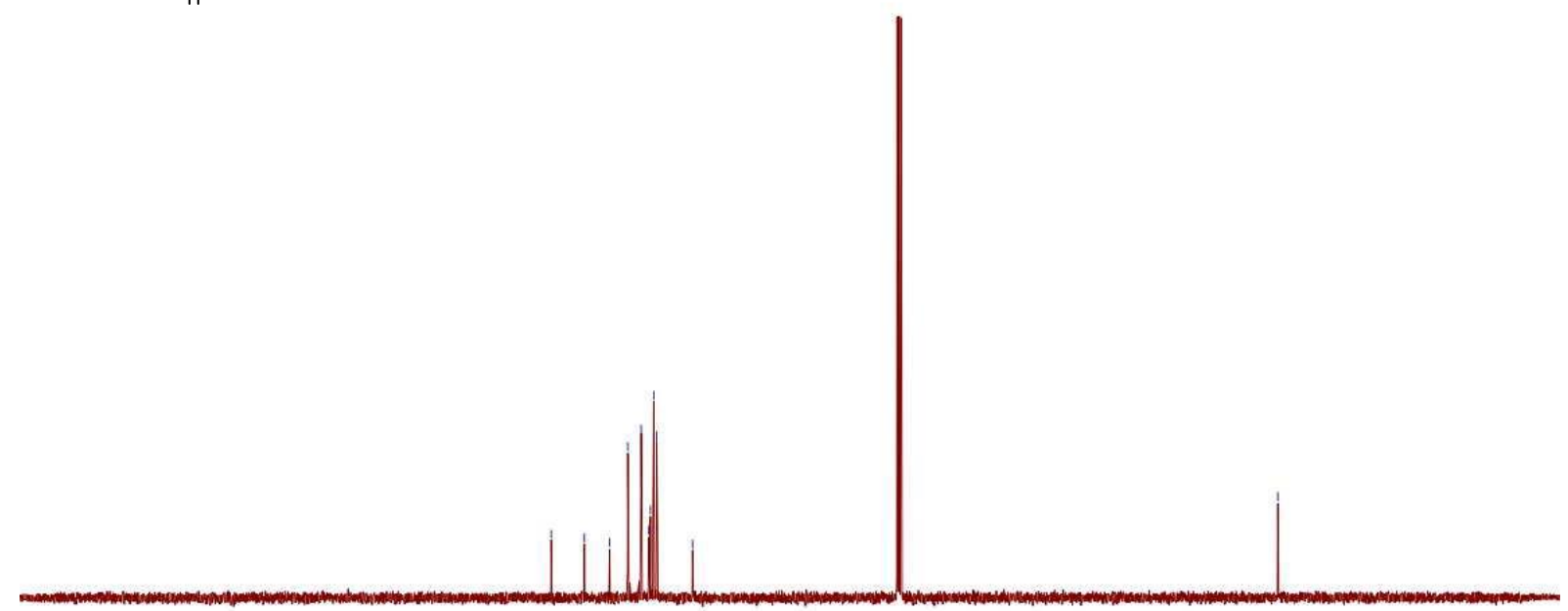

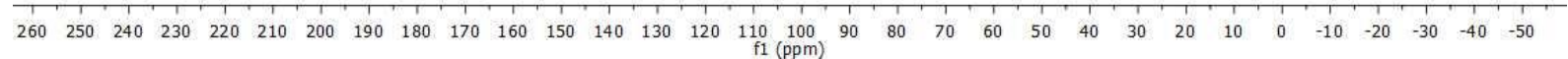


4d $\left(300 \mathrm{MHz}, \mathrm{CDCl}_{3}\right)$ :

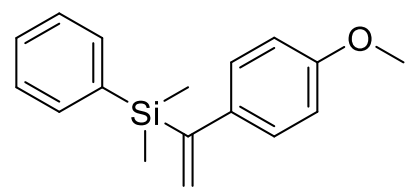

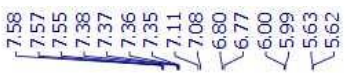

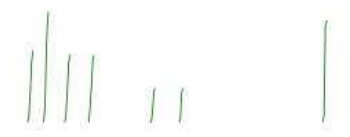

com

$\underset{\substack{m \\ i}}{m}$

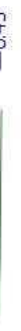

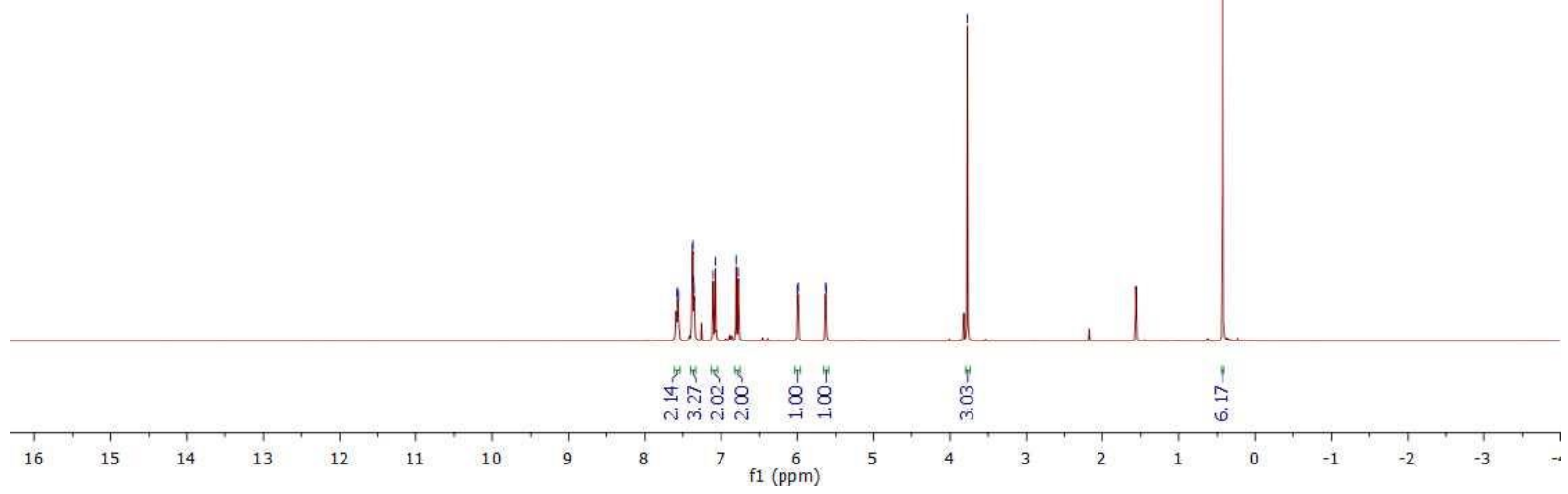

4d $\left(75 \mathrm{MHz}, \mathrm{CDCl}_{3}\right): \quad$ 年

$\frac{1}{\substack{1 \\ 0}}$

:

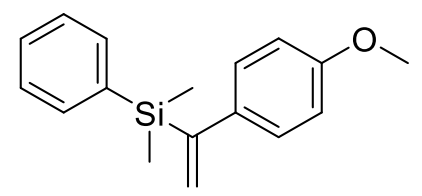

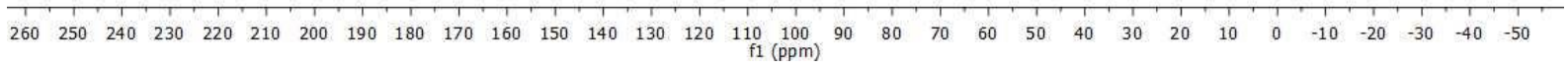

44 
4e $\left(300 \mathrm{MHz}, \mathrm{CDCl}_{3}\right)$ :

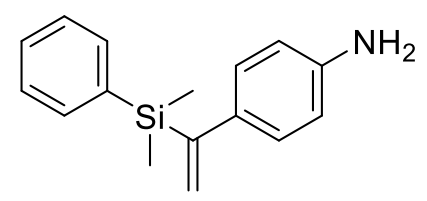

管

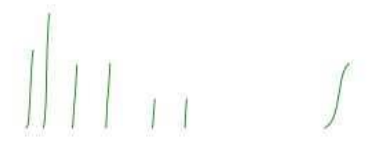

先

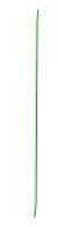

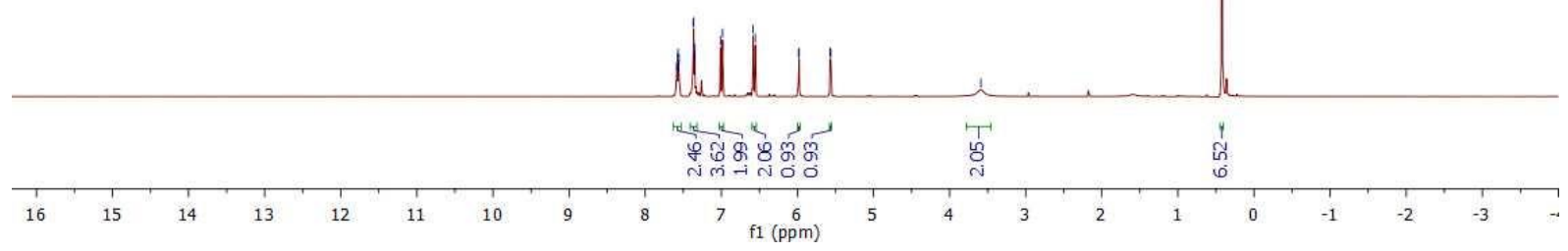
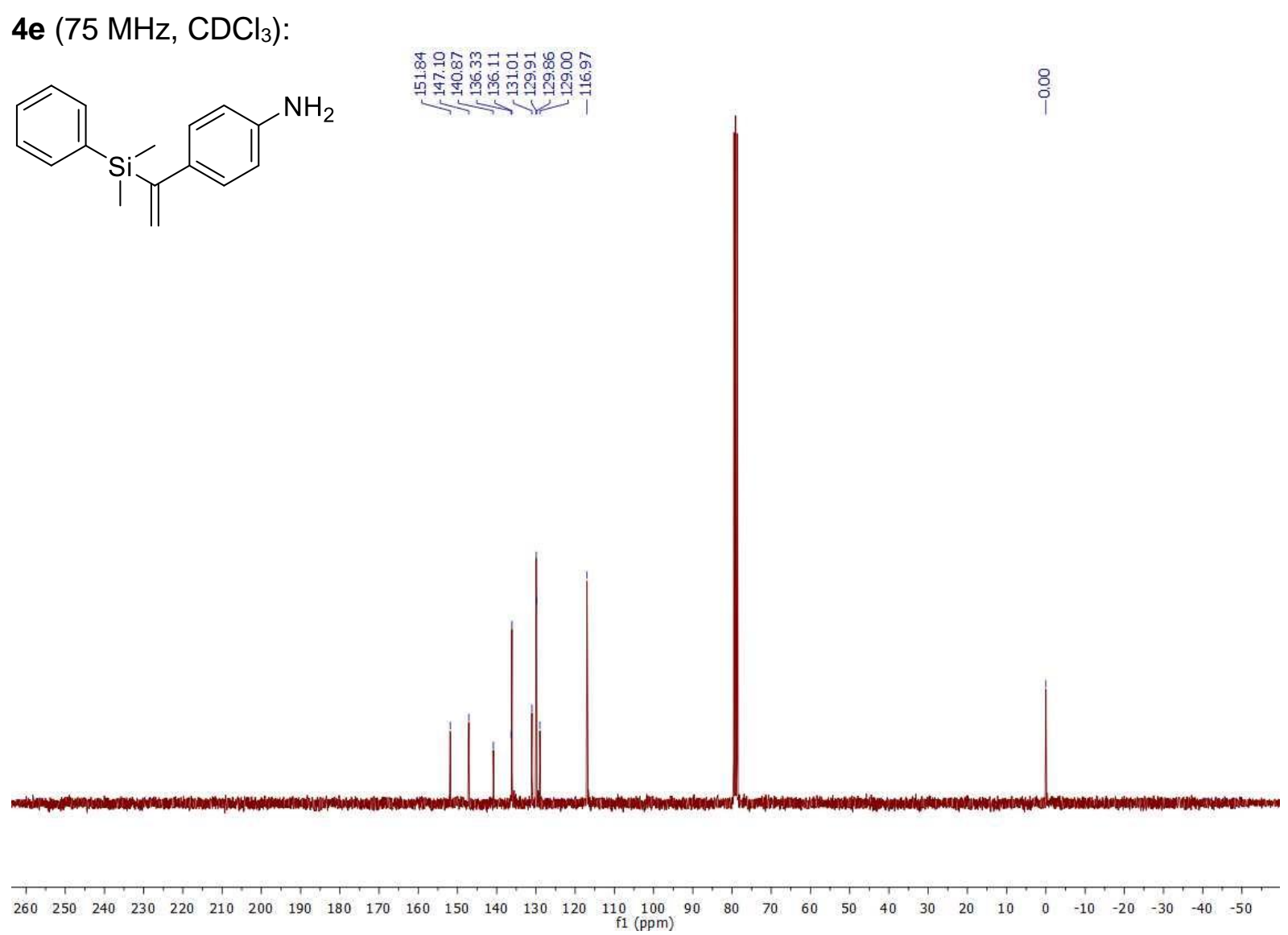

45 


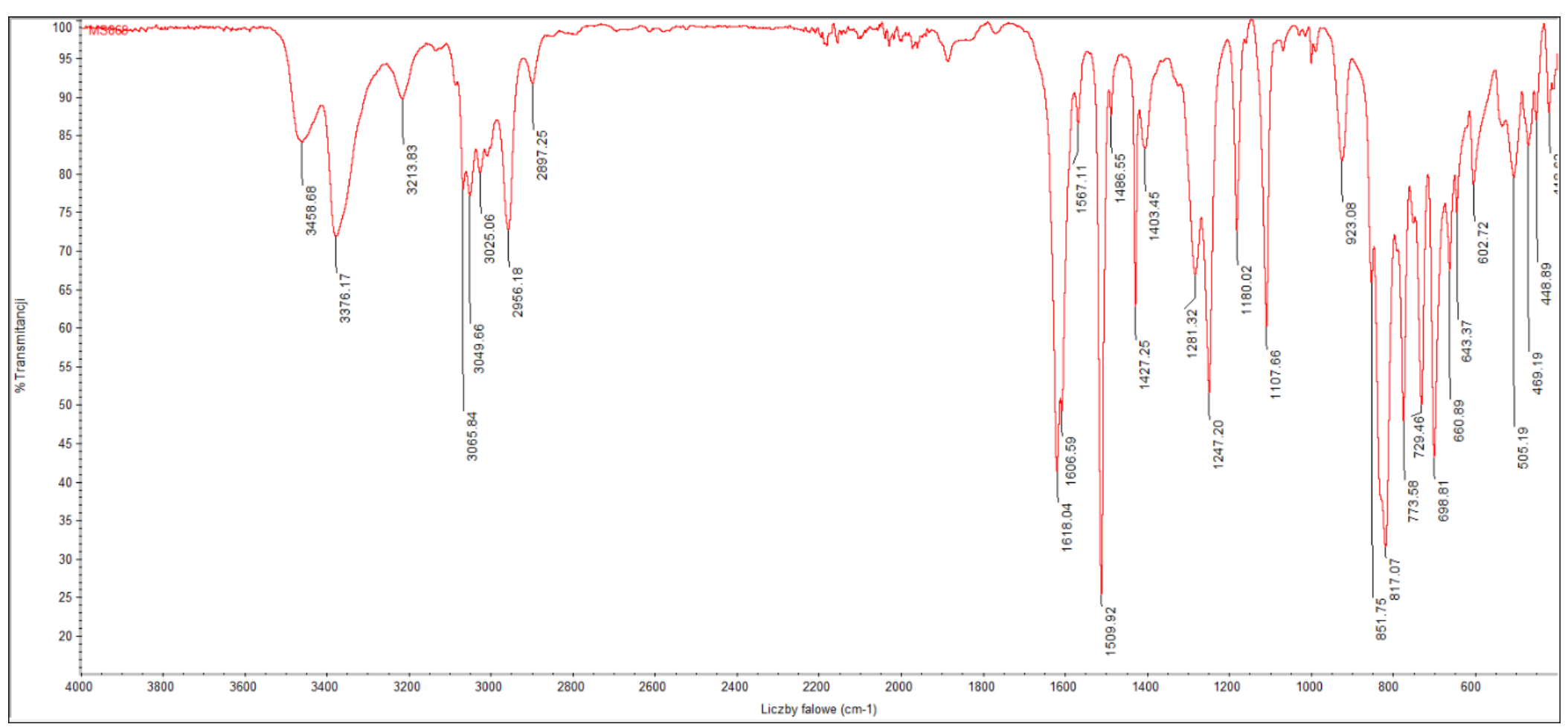


4f $\left(300 \mathrm{MHz}, \mathrm{CDCl}_{3}\right)$ :

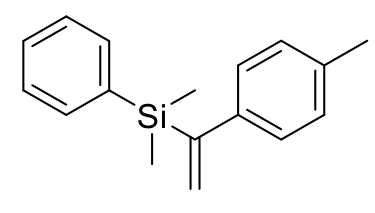

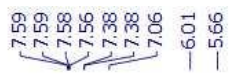

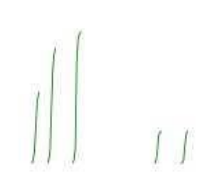

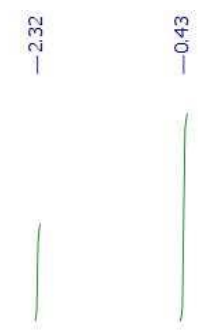

㴽

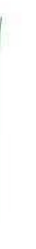

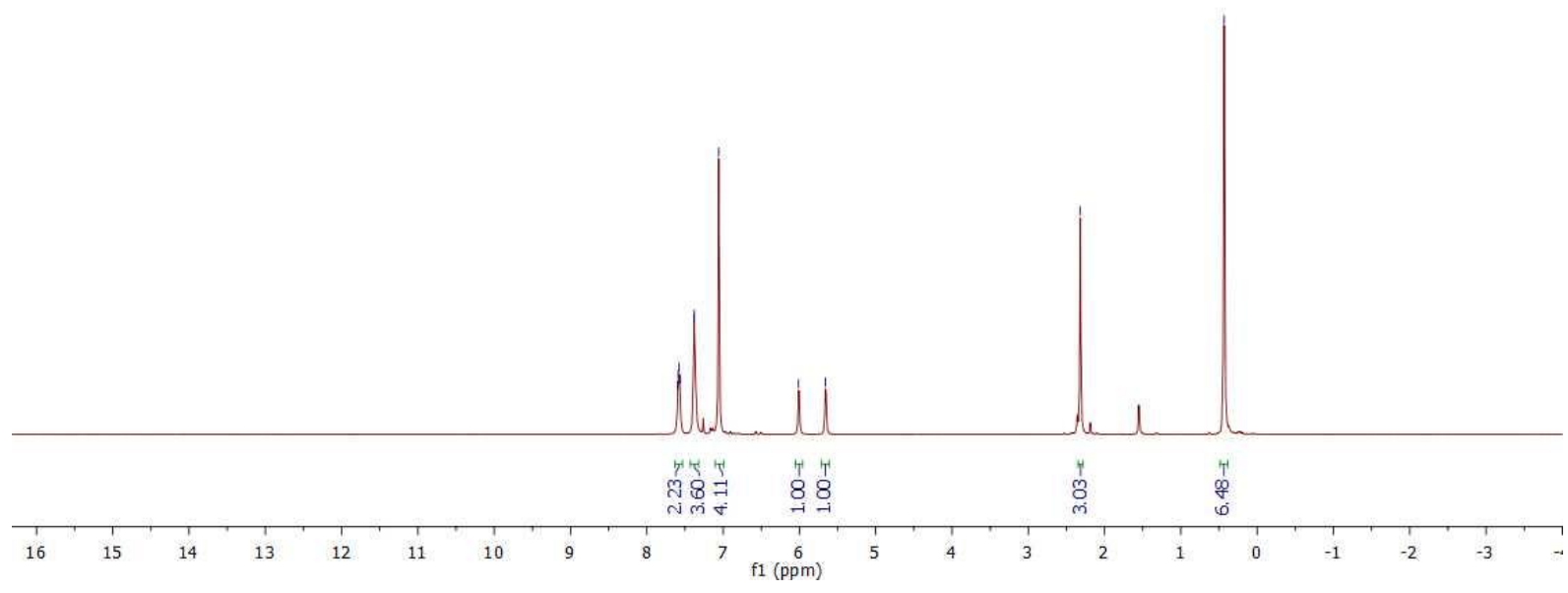

$4 f\left(75 \mathrm{MHz}, \mathrm{CDCl}_{3}\right)$ :

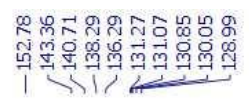

8
$\substack{n \\ 1}$
1
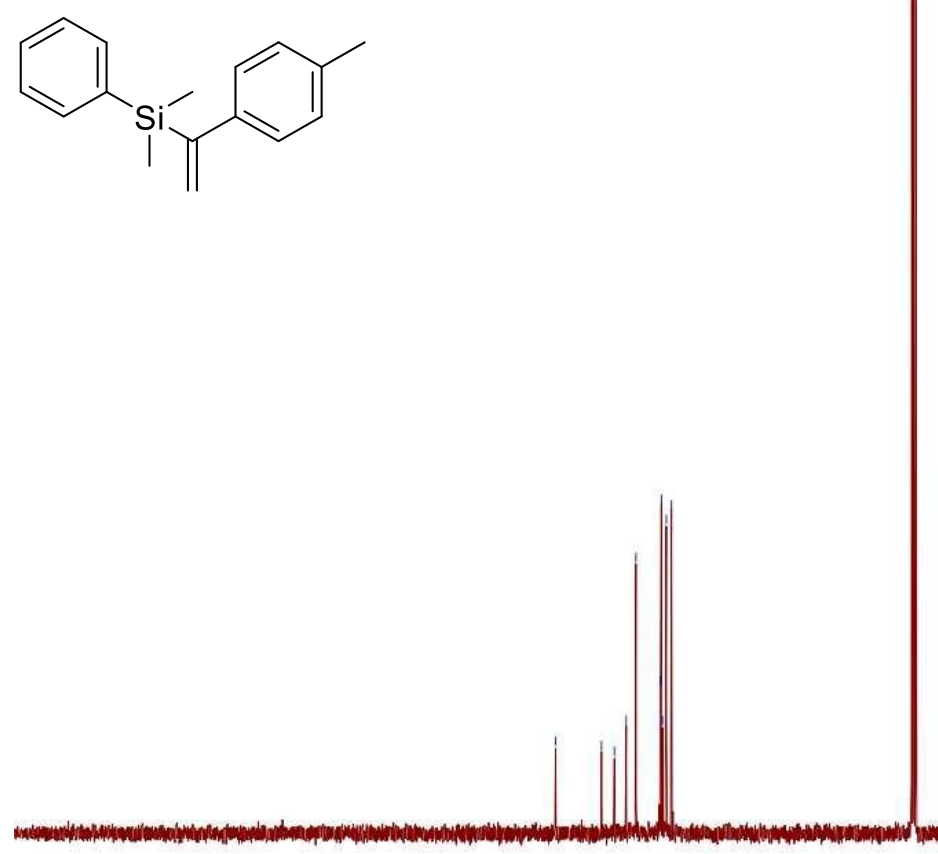

$\begin{array}{llllllllllllllllllllllllllllllllll}260 & 250 & 240 & 230 & 220 & 210 & 200 & 190 & 180 & 170 & 160 & 150 & 140 & 130 & 120 & 110 & 100 & 90 & 80 & 70 & 60 & 50 & 40 & 30 & 20 & 10 & 0 & -10 & -20 & -30 & -40 & -50\end{array}$

47 


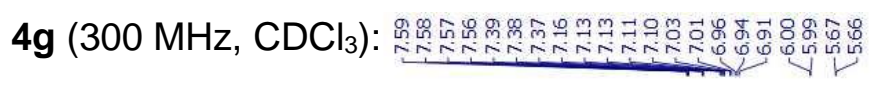
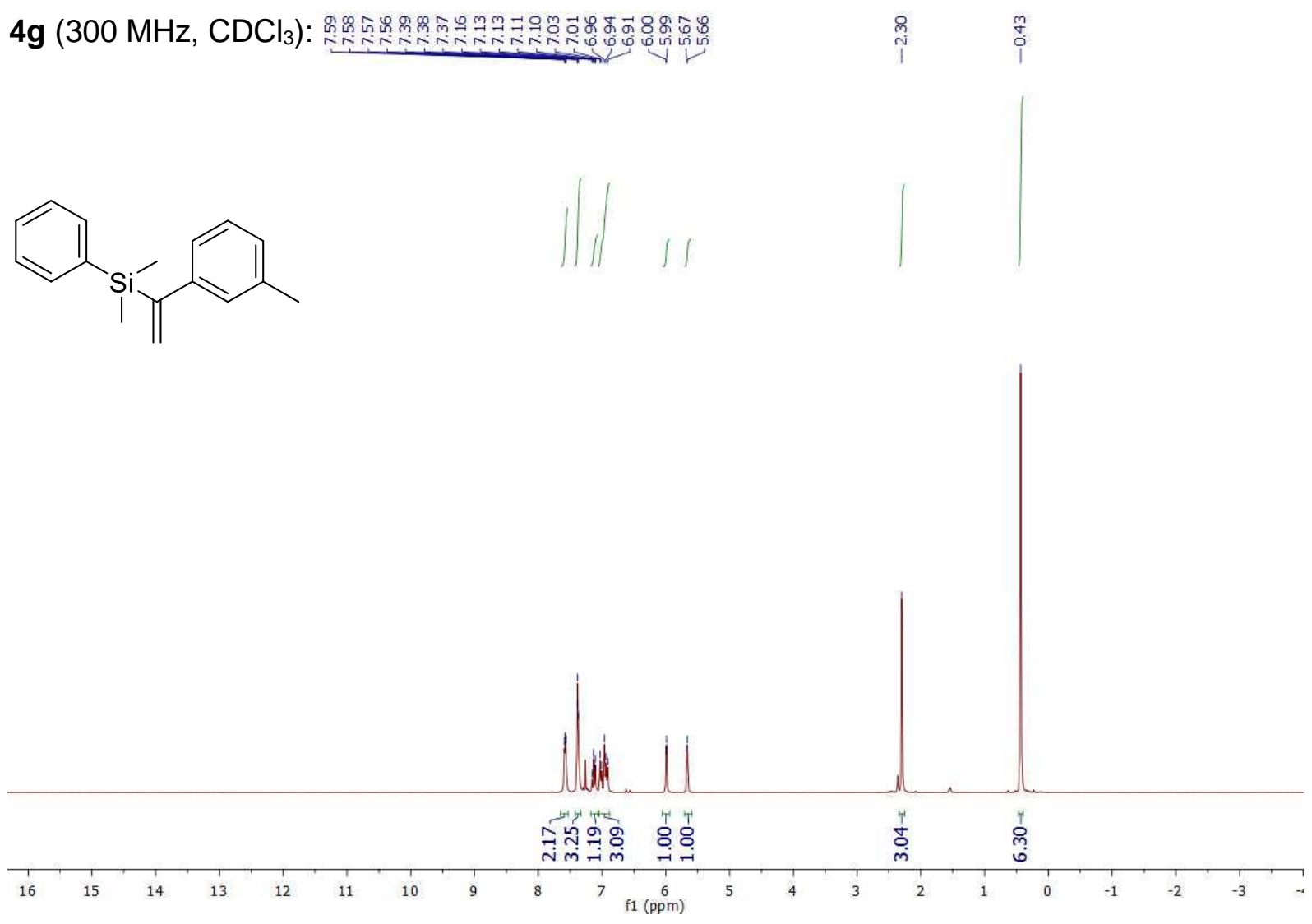

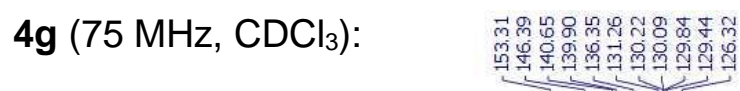

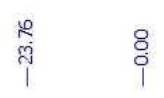
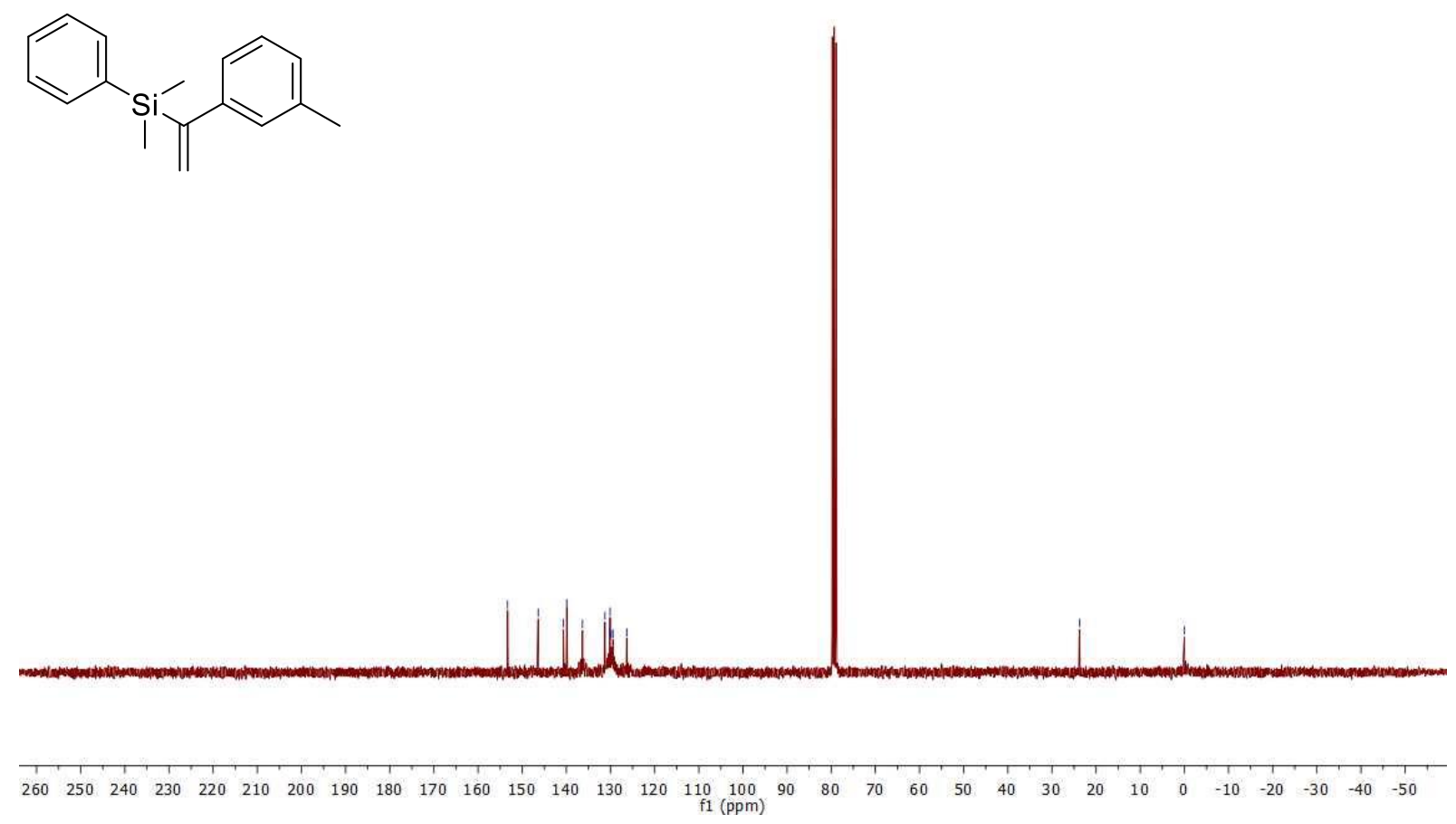

48 


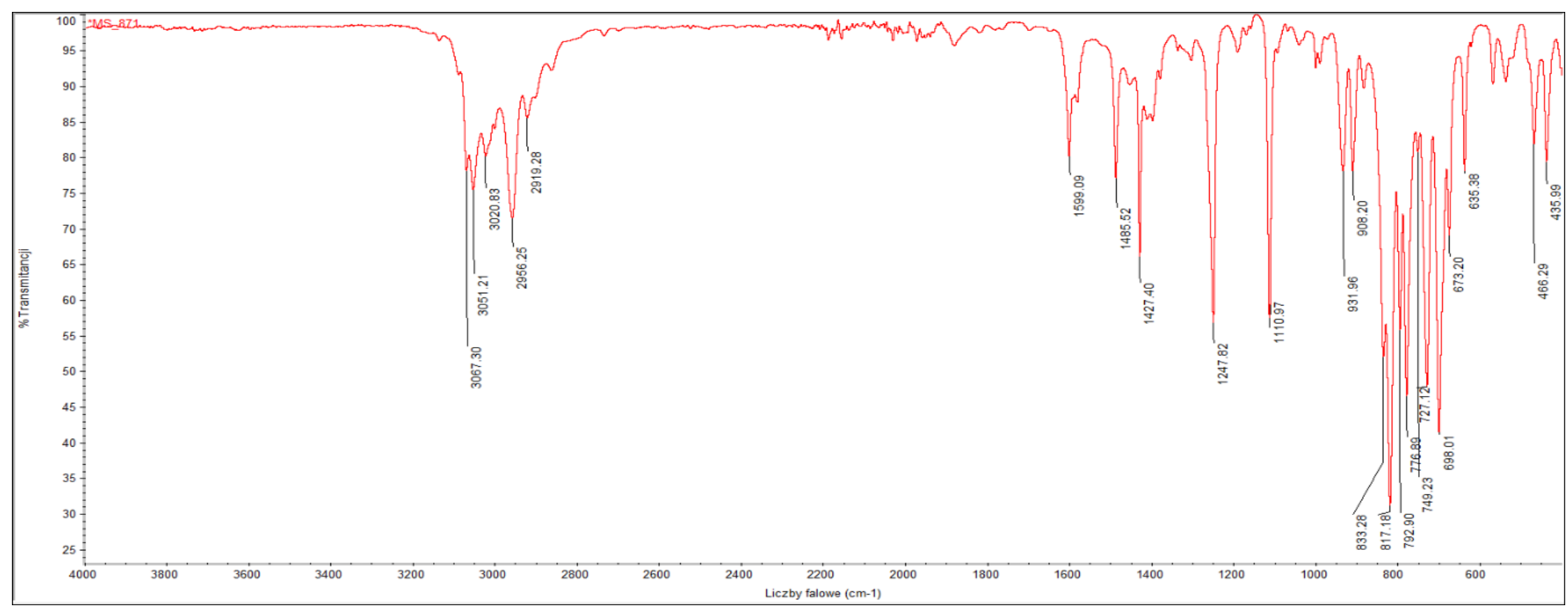


4h $\left(300 \mathrm{MHz}, \mathrm{CDCl}_{3}\right)$ :

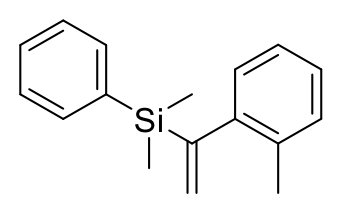

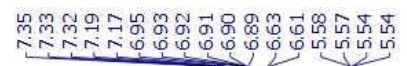

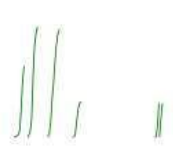

$\overrightarrow{\vec{T}}$

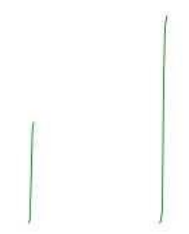

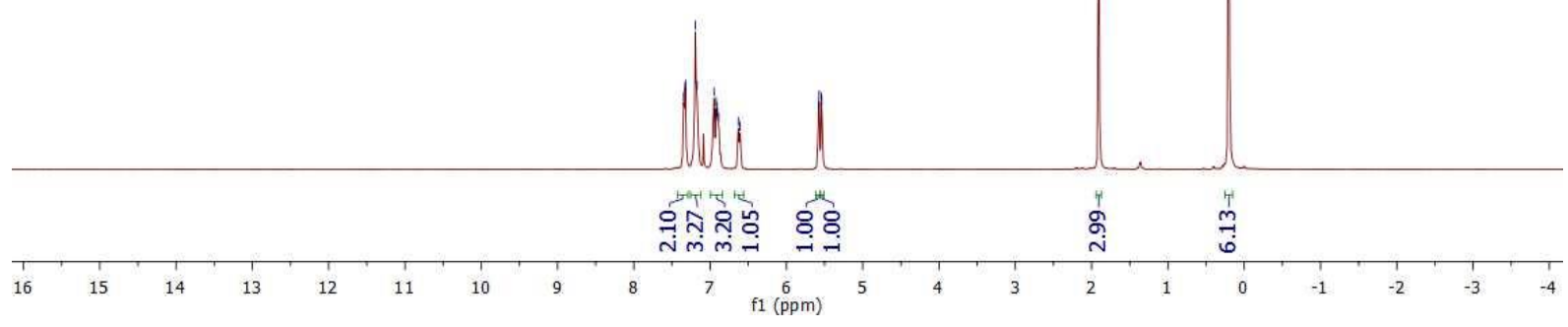

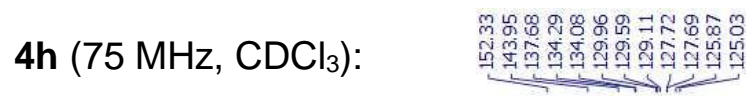

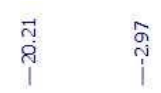
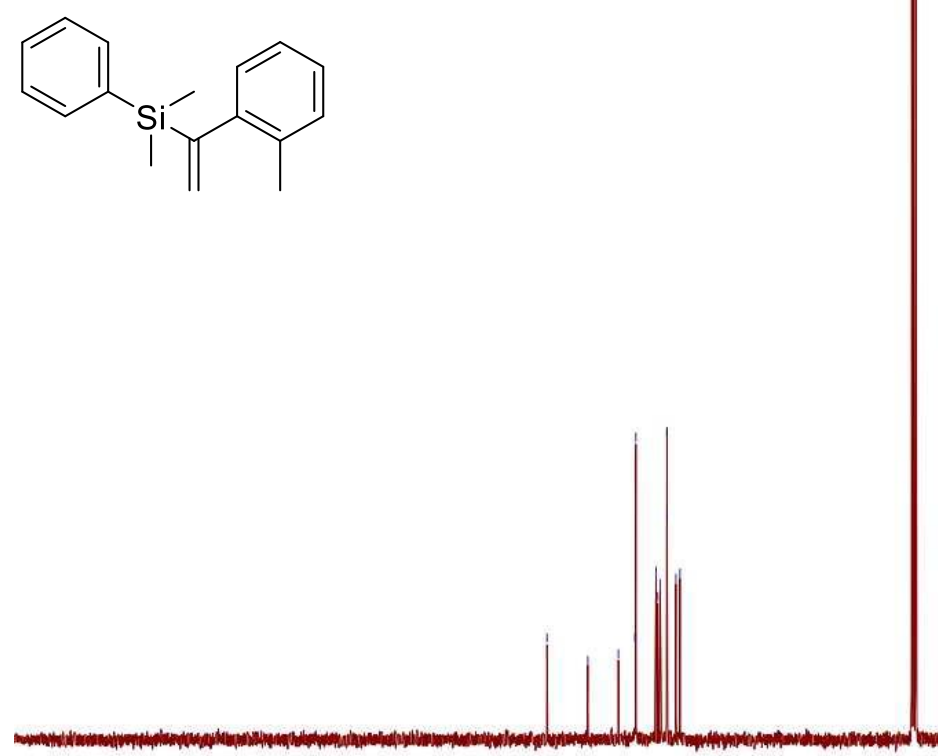

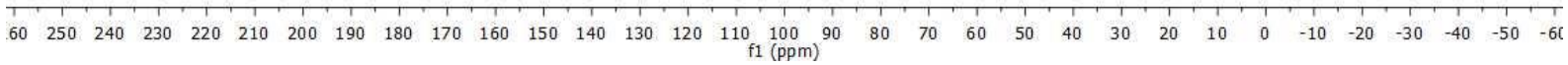

50 
4i $\left(300 \mathrm{MHz}, \mathrm{CDCl}_{3}\right)$ :

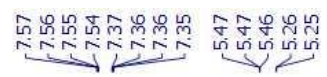

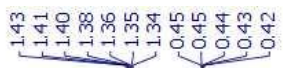
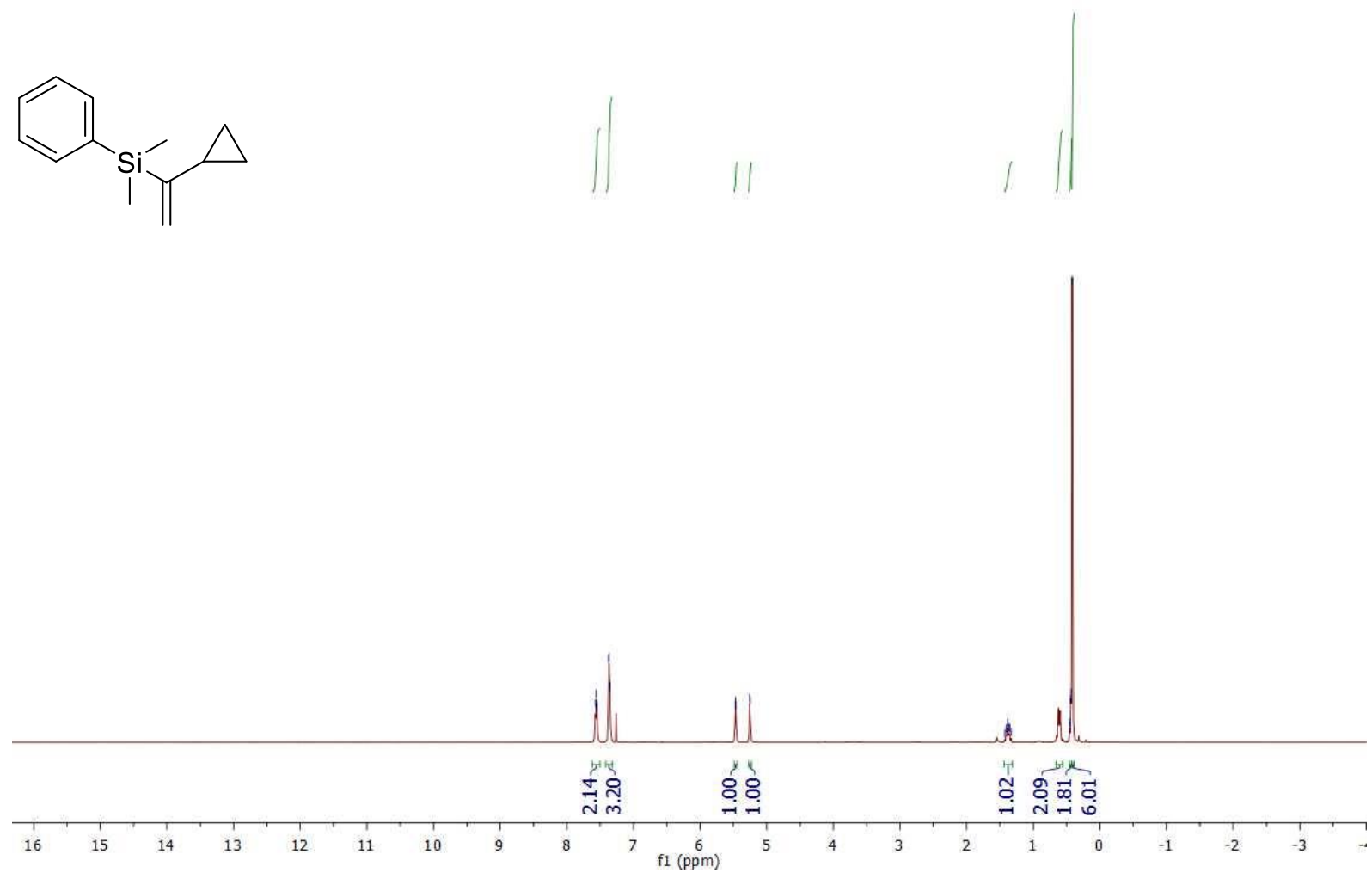

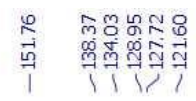

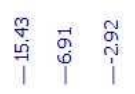

$4 \mathbf{i}\left(75 \mathrm{MHz}, \mathrm{CDCl}_{3}\right)$ :
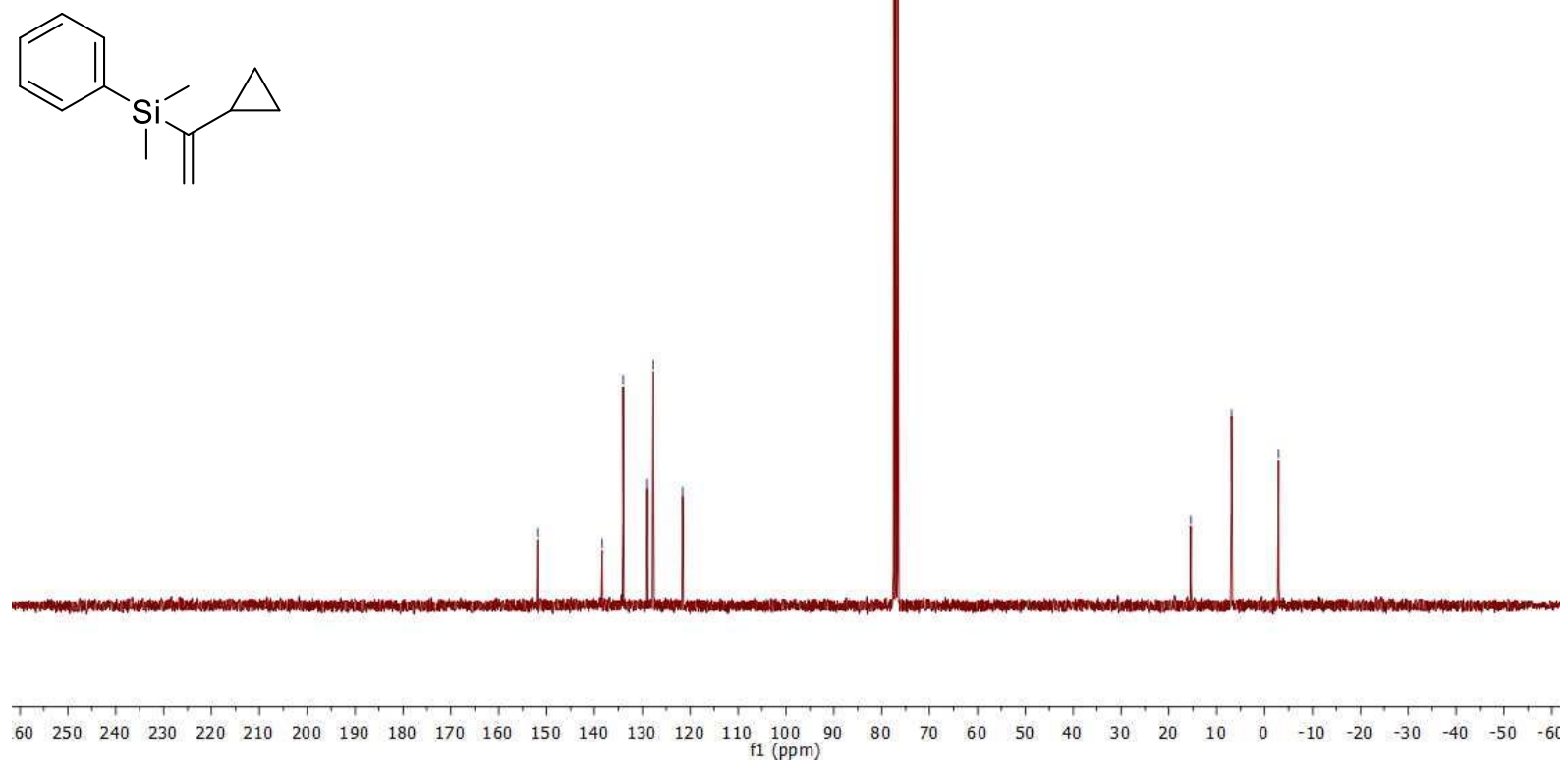

51 


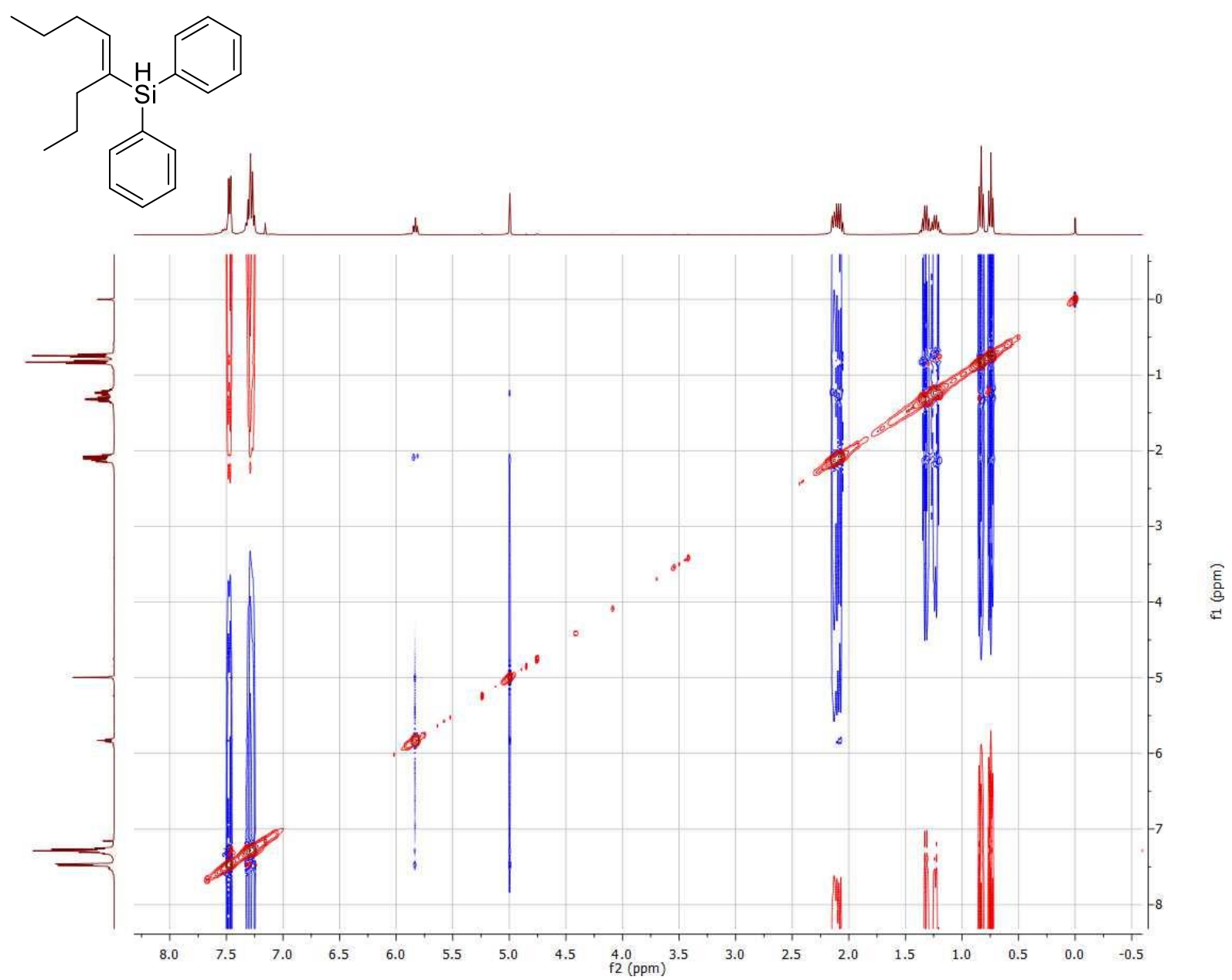

Deuterium labelled experiment $\left(300 \mathrm{MHz}, \mathrm{CDCl}_{3}\right)$ :<smiles>[2H]C=C(c1ccccc1)[SiH](c1ccccc1)c1ccccc1</smiles>

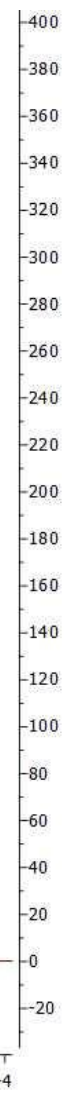

52 


\section{References:}

[1] Bocian, A,; Szymańska, M.; Brykczyńska, D.; Kubicki, M.; Wałęsa-Chorab, M.; Roviello, GN.; FikJaskółka, MA.; Gorczyński A.; Patroniak, V., Molecules. 2019, 24(17), 3173.

[2] Bocian, A.; Skrodzki M.; Kubicki, M.; Gorczyński, A.; Pawluć, P.; Patroniak, V. Appl. Catal. A, 2020, $602,117665$.

[3] Guo, J.; Lu, Z., Angew. Chem. Int. Ed. 2016, 55(36), 10835-10838.

[4] Zuo, Z.; Yang, J.; Huang, Z., Angew. Chem. Int. Ed. 2016, 55(36), 10839-10843.

[5] Guo, J.; Shen, X.; Lu, Z., Angew. Chem. Int. Ed. 2017, 56(2), 615-618.

[6] Skrodzki, M.; Zaranek, M.; Witomska, S.; Pawluć, P. Catalysts, 2018, 8, 618.

[7] Zhang, S.; Ibrahim, J. J.; Yang, Y., Org. Lett. 2018, 20, 19, 6265-6269.

[8] Song, L.; Feng, Q.; Wang, Y.; Ding, S.; Wu, Y-D.; Zhang, X.; Chung L. W.; Sun, J., J. Am. Chem. Soc. 2019, 141, 43, 17441-17451

[9] Cassani, M. C.; Brucka M. A.; Femoni, C.; Mancinelli, M.; Mazzanti A.; Mazzoni, R.; Solinas, G., New J. Chem., 2014,38, 1768-1779

[10] Gee, J. C.; Fuller, B. A.; Locket, H.-M.; Sedghi, G.; Robertson, C. M.; Luzyanin K. V., Chem. Commun., 2018, 54, 9450-9453.

[11] Rivero-Crespo, M. A.; Levya-Perez A.; Corma, A., Chem. Eur. J., 2017, 23(7), 1702-1708.

[12] Wang, P.; Yeo X.-L.; Loh P.-T., J. Am. Chem. Soc. 2011, 133, 5, 1254-1256. 